\title{
Alterations in Oral Mucosal Microbiota of Patients with Oral Potentially Malignant Disorders
}

\author{
Ph.D. Thesis
}

Dr. Gábor Sándor Decsi, DDS

\author{
University of Szeged \\ Doctoral School of Clinical Medicine
}

Supervisors:

Prof. Dr. habil. Katalin Nagy, DDS, Ph.D., DSc

Dr. habil. Krisztina Buzás, Ph.D.

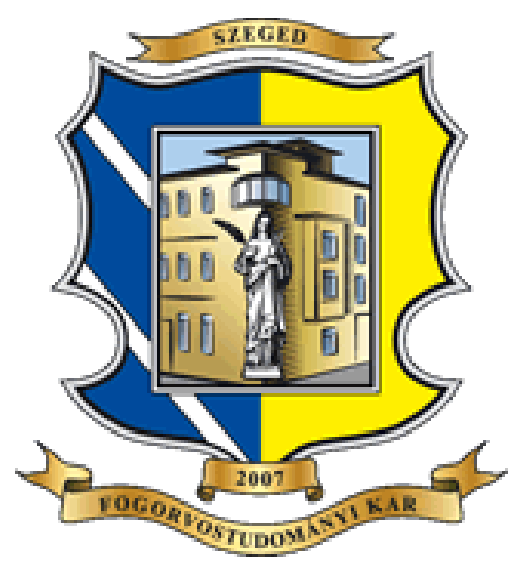

University of Szeged

Faculty of Dentistry

Szeged, Hungary

2020 


\section{Publications Providing the Basis of the Thesis}

I. Decsi G, Soki J, Pap B, Dobra G, Harmati M, Kormondi S, Pankotai T, Braunitzer G, Minarovits J, Sonkodi I, Urban E, Nemeth IB, Nagy K, Buzas K (2019) Chicken or the Egg: Microbial Alterations in Biopsy Samples of Patients with Oral Potentially Malignant Disorders. Pathol Oncol Res 25(3):1023-1033

Q2

\section{IF: 2,433}

II. Harmati M, Gyukity-Sebestyen E, Dobra G, Terhes G, Urban E, Decsi G, MimicaDukić N, Lesjak M, Simin N, Pap B, Nemeth IB, Buzas K (2017) Binary mixture of Satureja hortensis and Origanum vulgare subsp. hirtum essential oils: in vivo therapeutic efficiency against Helicobacter pylori infection. Helicobacter 22(2)

Q1

\section{IF: 4,123}

III. Hettmann A, Demcsák A, Decsi G, Bach Á, Pálinkó D, Rovó L, Nagy K, Takács M, Minarovits J (2016) Infectious Agents Associated with Head and Neck Carcinomas. Adv Exp Med Biol 897:63-80

Q2

IF: 0,65 


\section{Table of Contents}

Publications Providing the Basis of the Thesis

Table of Contents

Tables and Figures

List of Abbreviations

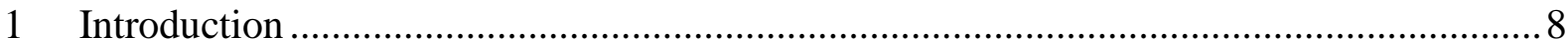

1.1 Oral potentially malignant disorders: a group of disorders of diverse etiologies ........8

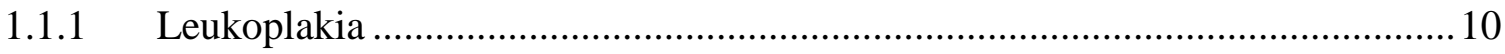

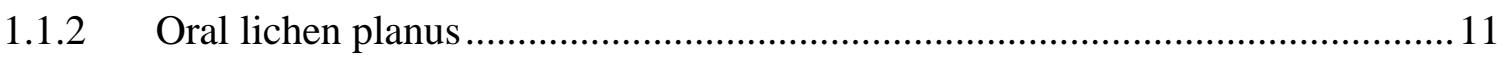

1.2 Microbiota, microbiome, metagenome of healthy oral mucosa …......................... 12

1.3 Evidences for connection between bacteria and OSCC ....................................... 14

$1.4 \quad$ Indication and technical basis of our experimental setup ..................................... 17

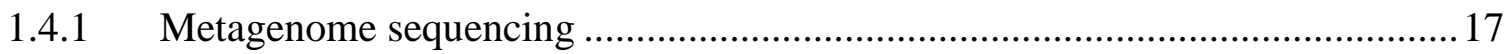

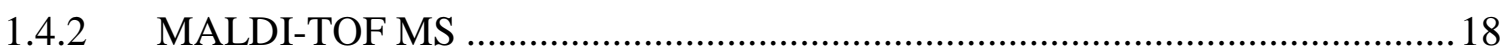

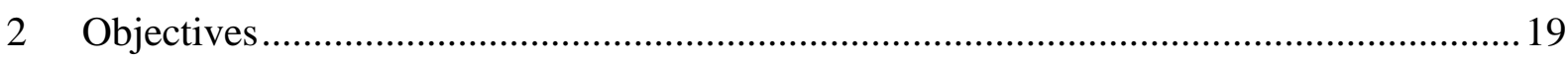

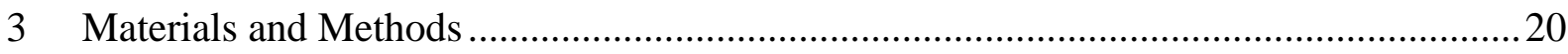

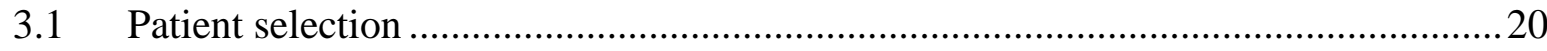

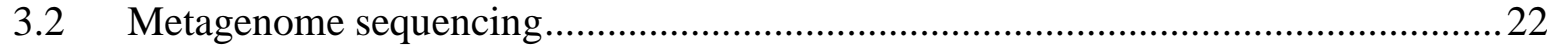

3.2.1 Tissue sample collection for metagenome sequencing and histopathological

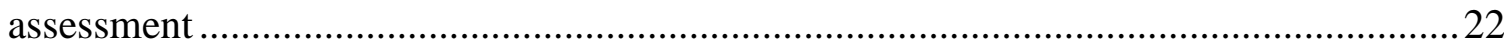

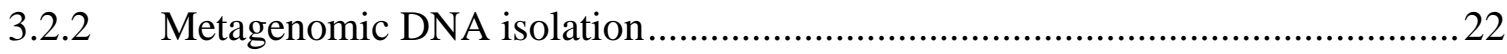

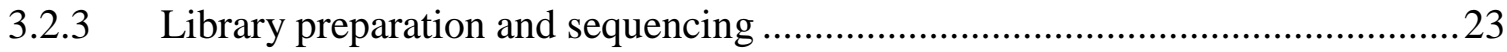

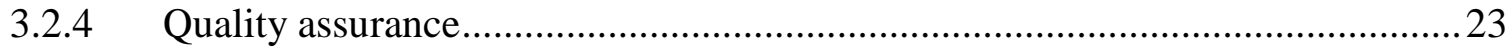

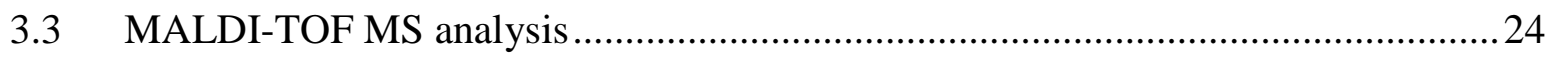

3.3.1 Swab sample collection for microbiological culture and identification by

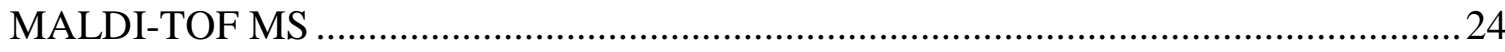

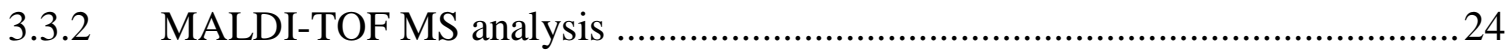

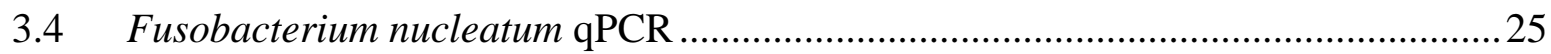

3.4.1 Fusobacterium nucleatum $\mathrm{qPCR}$ process....................................................25 


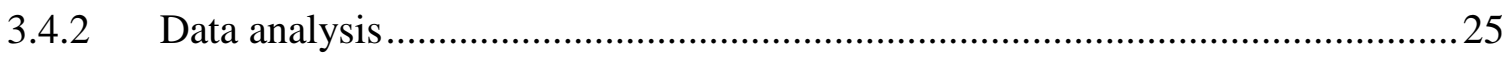

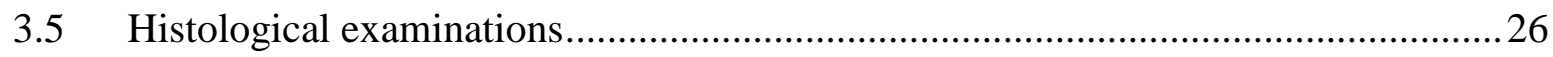

3.5.1 Histopathological assessment of the tissue samples collected from OPMD

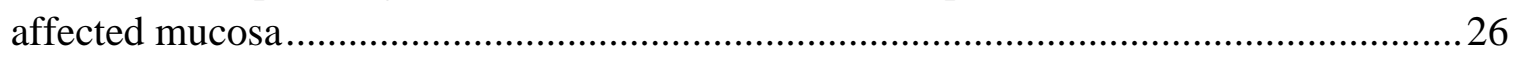

3.5.2 Examination of mucosal lesions caused by Helicobacter pylori in a murine

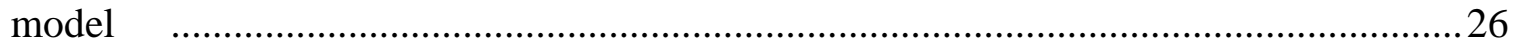

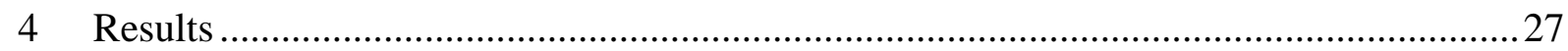

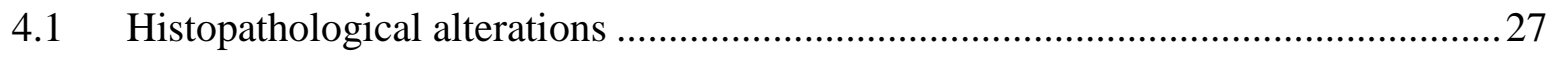

4.1.1 Histopathological changes in OPMD tissues..................................................27

4.1.2 Histopathological changes in Helicobacter pylori infected gastric mucosa.......2 28

4.2 Analysis of microbiome/microbiota in biopsy and swab samples from OPMD lesions

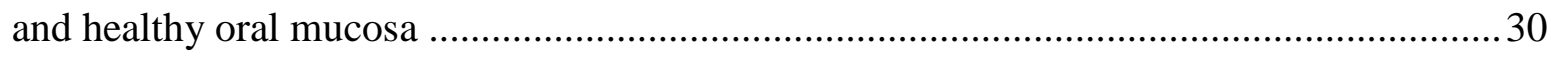

4.2.1 Microbiome analysis by metagenome sequencing …………………………...... 30

4.2.2 Analysis of microbiota by MALDI-TOF MS …………………………........... 36

4.2.3 Quantitation of Fusobacterium nucleatum in oral swab samples by qPCR ........37

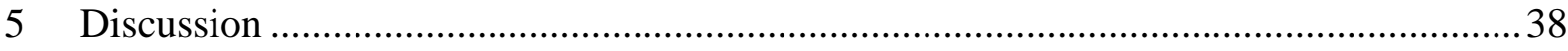

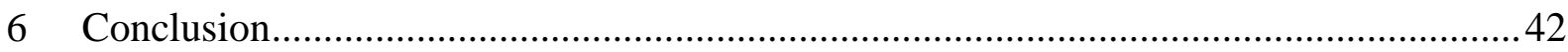

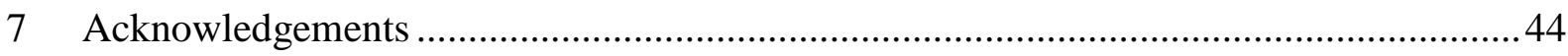

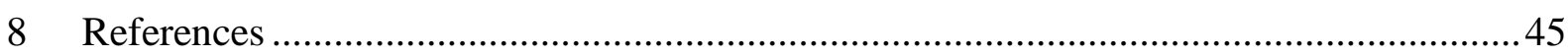

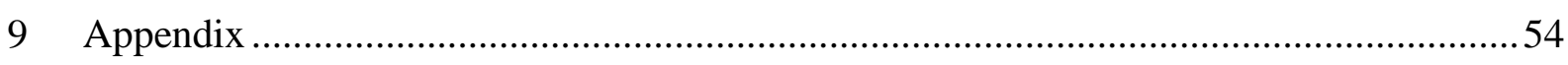




\section{Tables and Figures}

\section{Tables}

Table 1 Presence of HPV DNA in OPMD and OSCC lesions in literature 9

Table 2 Brief medical history of patients. 21

Table 3 Histopathological characteristics of the biopsies 27

Table 4 Comparison of bacterial diversity in healthy mucosa and OPMD lesion 34

\section{Figures}

Fig. 1 Representative pictures from OPMD tissue samples 28

Fig. 2 Long-term H. pylori infection in mice 29

Fig. 3 Distribution of taxonomic domains 30

Fig. 4 Bacterial diversity 31

Fig.5 Comparison of the detected bacterial species 32

Fig. 6 Ratio of Streptococci in the mucosal lesion 33

Fig. 7 Fusobacterium nucleatum in the mucosal lesion 35

Fig. 8 MALDI-TOF MS analysis 36

Fig. 9 Fusobacterium nucleatum-specific PCR of the healthy tissue and the OPMD 37 


\section{List of Abbreviations}

2-5 DHB - 2,5-dihydroxybenzoic acid

BHIB - brain heart infusion broth

CFU - colony forming unit

CTAB - cetyltrimethilammonium bromid

CSF - cerebrospinal fluid

DNA - deoxyribonucleic acid

EBV - Epstein-Barr virus

EDTA - ethylendiamintetraacetic acid

ePCR - emulsion polymerase chain reaction

HCCA - $\alpha$-cyano-4 hydroxy-cinnamic acid

HE - hematoxylin-eosin

HNCC - head and neck carcinoma

HPA - 3-hydroxypicolinic acid

HPV - human papilloma virus

$\mathbf{L P}$ - lichen planus

MALDI-TOF MS - matrix-assisted laser desorption ionization- time of flight mass spectrometry

MDR 1 gene - multidrug resistance 1 gene

MG-RAST - metagenome rapid annotation using subsystem technology

MT - malignant transformation

OLP - oral lichen planus

OPMD - oral potentially malignant disorders

OSCC - oral squamous cell carcinoma 
PCR - polymerase chain reaction

PGM - personal genome machine

PVL - proliferative verrucous leukoplakia

QC - quality control

qPCR - quantitative polymerase chain reaction

RNA - ribonucleic acid

RT-PCR - real time polymerase chain reaction

SDS - sodium dodecyl sulphate

STD - sexually transmitted disease

TE - Tris-EDTA

TTV - torque teno virus

WHO - World Health Organisation 


\section{Introduction}

\subsection{Oral potentially malignant disorders: a group of disorders of diverse etiologies}

Although numerous scientific data demonstrates bacterial colonization of cancerous tissue, in most cases the role of bacteria in carcinogenesis remains to be established. In humans, Helicobacter pylori is the only bacterium recognised as the causative agent of a malignant disease, gastric adenocarcinoma [1]. Thus, Helicobacter pylori has been classified as a class I carcinogen.

As to the carcinomas of the head and neck region, the role of oral microbes in the development of oral potentially malignant disorders (OPMDs) and oral squamous cell carcinoma (OSCC) is periodically reevaluated, since OPMD or OSCC may develop in the absence of the traditional risk factors like smoking, alcohol consumption and betel nut use [24]. While the microbiological background of oral squamous cell carcinoma was intensively studied in the last two decades, less attention has been paid to OPMD in this respect [5]. OPMD is a group of disorders of diverse etiologies, frequently associated with tobacco consumption and mutations in the DNA of oral epithelial cells. A fraction of OPMDs undergoes clinical and histomorphological alterations that lead to the development of OSCC. In 1978, working group of WHO classified 'oral precancer' into 'lesions' and 'conditions' with following definitions: A precancerous lesion is 'a morphologically altered tissue in which oral cancer is more likely to occur than in its apparently normal counterpart'. A precancerous condition is ,a generalized state associated with a significantly increased risk of cancer". The current Working Group of WHO (2005) does not favour subdividing precancer to lesions and conditions and the consensus view was to refer to all clinical presentations that carry a risk of cancer under the term „oral potentially malignant disorders” (OPMD) to reflect their widespread anatomical distribution. These disorders include leukoplakia, erythroplakia, oral lichen planus, submucous fibrosis, and actinic cheilitis [6, 7]. In addition, inherited cancer syndromes such as xeroderma pigmentosum and Fanconi's anemia are also associated 
with an increased incidence of malignant tumors, among them oral carcinoma [7]. In most type of OPMD we know the etiological factors of the disease, whereas in other cases the etiology is only partially clear (oral lichen planus), or not clear yet (proliferative verrucous leukoplakia). The most intensely studied infectious agent implicated in the development of OPMD and OSCC is HPV (human papilloma virus). There are demonstrative results in the literature regarding the presence of HPV in OPMD and OSCC lesions (Table 1). Table 1. also shows, however, that in a significant fraction of the OPMD and OSCC samples analysed, HPV could not be detected. For this reason, we looked for bacteria, another potential etiological factor, in OPMD lesions [8].

Table 1 Presence of HPV DNA in OPMD and OSCC lesions in literature [8]

\begin{tabular}{lcc}
\hline \multicolumn{1}{c}{ Disorder/disease } & $\begin{array}{c}\text { Presence of HPV DNA } \\
\text { (in \% of examined } \\
\text { samples) }\end{array}$ & \\
\hline Lichen planus & $23-32,8 \%$ & {$[98,99]$} \\
$\begin{array}{l}\text { Leukoplakia/proliferative verrucous } \\
\text { leukoplakia }\end{array}$ & $25,4-40,9 / 26,5-40,8 \%$ & {$[98-101]$} \\
Erythroplakia & $54,5 \%$ & {$[102]$} \\
Oral squamous cell carcinoma & $47,7 \%$ & {$[99,102]$} \\
\hline
\end{tabular}

Leukoplakia and oral lichen planus (OLP) are the most frequent OPMDs in our clinical practice at the Department of Oral Medicine, Faculty of Dentistry, University of Szeged. Both disorders appear as whitish coloured hyperkeratosis, similarly to some other OPMDs. From the point of view of clinical differential diagnostics, this group of OPMDs belongs to a „Potentially malignant and malignant” subgroup (leukoplakias, submucosal fibrosis, lichen planus, lupus erythematosus, epidermoid carcinoma, verrucous carcinoma) of so called „White lesions”. The group of „White lesions” also includes additional subgroups, such as the „Congenital”, „Aquired infectious and non-infectios”, and „Other” subgroups containing diseases with different etiologies. The possible cause of the whitish colour of "White lesions" could be hyperkeratosis, pseudomembranous infection or inflammation. Due to the heterogenity of diseases with the same clinical feature, clinical diagnostics of white lesions could be very difficult. Thus, quite frequently, pathological or microbiological support is needed to make a proper diagnosis, prognosis and treatment plan. [9] 


\subsubsection{Leukoplakia}

Leukoplakia is defined as "A white plaque of questionable risk having excluded (other) known diseases or disorders that carry no increased risk for cancer". Leukoplakia is six times more common among smokers than among non-smokers [7]. These lesions are divided into homogenous (simplex) and non-homogenous types. The non-homogenous types based on the cellular variability are verrucous leukoplakia, nodular leukoplakia and erythroleukoplakia [9]. Proliferative verrucous leukoplakia (PVL) is a subtype of verrucous leukoplakia that shows resistance to treatment and a high rate of malignant transformation. It is more frequent among elderly women, in many cases without smoking and consumption of alcohol in the anamnesis. Distinct histopathological changes, like hyperkeratosis with or without dysplasia, may accompany the transition of PVL to verrucous hyperplasia and verrucous carcinoma [7]. There are conflicting results regarding the association of leukoplakia and human papillomavirus (HPV) infection [7, 10]. The role of torque teno virus (TTV) [11-13], EpsteinBarr virus (EBV) [14] and Candida albicans [10, 15-19] in leukoplakia development and carcinogenesis remains to be clarified, too. Additionally, it was suggested that specific bacterial infections like Helicobacter pylori [20], or the intracellular Mycoplasma salivarium [21] could also be involved in this process.

As to the putative role of bacterial infection in oral carcinogenesis, one may speculate that it could be a process analogous to Helicobacter pylori infection of stomach which predisposes to the development of gastric cancer. Thus, an in vivo murine model of Helicobacter pylori induced mucosal lesions could potentially mimic the lesions caused by other bacteria or bacterial communities on oral mucosal surfaces (see 4.1.2). As a matter of fact, a disbalance in the oral microbial flora may play a role in the development of leukoplakia and carcinogenesis, as suggested by the association of periodontal inflammation with leukoplakia [22] and a changing bacterial flora in the saliva and on the oral mucosal surfaces of patients with OPMD [23, 24]. 


\subsubsection{Oral lichen planus}

Lichen planus (LP) is a chronic, idiopathic, inflammatory disease of the oral mucosa or the skin, presenting as a white lesion when it affects the oral cavity (oral lichen planus, OLP). A crucial aspect of the pathomechanism of OLP is the accumulation of CD8+ T lymphocytes under the basal cell layer of the oral mucosa, which causes DNA damage and keratinocyte apoptosis by antigen-specific cell-mediated immune response. CD8+ T cells degrade the basement membrane as well [25-28]. According to the most accepted hypothesis, chronic stimulation from inflammatory and stromal cells provides the initial signal which leads to the uncontrolled growth of epithelial cells. Additionally, oxidative stress induced DNA damage could also lead to neoplastic changes, but the initial event leading to this signal cascade activation has not been characterized yet. Based on several lines of evidence, viral, fungal, and bacterial antigens have all been suggested [29-37] as a potential initiating factor in LP. If there is a relationship between the bacterial flora and OLP, the question is whether the trigger area is in the oral cavity, or at another area of the body, such as the skin and the genitals, the gastrointestinal tract, the larynx or the eyes. If oral bacterial infection could initiate OLP development, it is not clear whether a single bacterial species could initiate the OLP transformations, or the interaction of several species elicits this process. Additionally, the disturbed balance of the normal bacterial flora could also be involved in the initial steps of OLP activation. 


\subsection{Microbiota, microbiome, metagenome of healthy oral mucosa}

The term „Microbiota” means the assemblage of microorganisms present in a defined environment. "Metagenome" is the collection of genomes and genes from the members of a microbiota. „Microbiome” refers to the entire habitat, including the microorganisms (bacteria, archaea, lower and higher eurkaryotes, and viruses), their genomes (i.e., genes), and the surrounding environmental conditions [38]. It is worth to note that there is an important difference between the cultivation-based and the direct sequencing methods used for the analysis of microbiota: cultivation based techniques detects only a subset of microbes, whereas it is possible to detect both cultivable and non-cultivable taxa using next generation sequencing. Different regions of oral cavity provide quite different circumstances for microbial biofilm formation. Accordingly, the composition of microbiomes varies from habitat to habitat. The human oral microbiota is changing during the life, too [39]. We are interested in our study for the microbiota of bucca and tongue, because the examined diseases develop frequently in firmly and loosely attached mucosa of buccal, gingival and tongue surfaces, frequently in middle or old age.

To compare bacteria of OPMD tissues with bacteria of healthy oral mucosa, we looked for information in current literature about oral bacteria in health in childhood, adulthood, and in case of an isolated population. Papaioannou et al. described that the soft tissue samples were dominated in childhood by streptococci, particularly Streptococcus mitis (18.7\%) and Streptococcus oralis (9.5\%) as well as Streptococcus salivarius (9.5\%) [39]. The profiles of the tongue dorsum and saliva were characterized by high proportions of Prevotella melaninogenica $(5.7 \%$ and $5.6 \%$, respectively) and S. salivarius (10.9\% and 10.7\%, respectively). Periodontal pathogens were found at very low counts and proportions at all sample locations. A. actinomycetemcomitans was presented in all children, albeit in low numbers and proportions. Several species showed changes in proportions with age. The Actinomyces species showed increased proportions with age at the saliva and tongue locations. Streptococci also increased in proportion with age at most sample locations, particularly Streptococcus gordonii and Streptococcus sanguinis. T. forsythia, P. gingivalis, $T$. denticola as a group showed a significant increase in proportion with age. $P$. gingivalis 
showed a higher dissemination in the oral cavity (i.e. more positive habitats) in the older age groups [39].

Zaura et al. characterized the oral bacterial communities of healthy adults. They found - as a „core microbiome”- fifteen taxa: the genera Streptococcus, Neisseria, Corynebacterium, Rothia, Actinomyces, Haemophilus, Prevotella, Fusobacterium, Granulicatella, Capnocytophaga, representatives of the Veillonellaceae, Neisseriaceae and Pasteurellaceae families, the Bacteroidales order and unclassified Firmicutes. Unclassified Bacteria and an additional four taxa were found in two third of samples: genus Porphyromonas, Leptotrichia, TM7 genera incertae sedis and Campylobacter. A few sequences dominated each individual microbiome. Three of the sequences were found across all samples: two Veillonellaceae family members (phylum Firmicutes) and one Fusobacterium genus member (phylum Fusobacteria). Fusobacteria mediate coaggregation of microbiota and serve as a structural component of both healthy and disease-associated dental plaque. Firmicutes dominated the cheek mucosa in two third of volunteers [40].

Oral microbiota varies in different human populations, and it is affected by environmental factors. Cheek microbiota of isolated amerindians show similar numbers of families to nonamerindians (30 in Amerindians and 33 in non-Amerindians), but with higher richness of genera in non-Amerindians (177) than in Amerindians (51). Amerindians shared only $62 \%$ of the families and $23 \%$ of the genera with non-Amerindians. The Amerindians carried marker bacteria that were not reported in previous studies, such as soil bacteria Gp1, and the xylanolytic Xylanibacter (Prevotellaceae), as well as Phocoenobacter (Pasteurellaceae), first described in a porpoise, in addition to unidentified members of the phyla Bacteroidetes, Firmicutes and Proteobacteria [41]. 


\subsection{Evidences for association between bacteria and OSCC}

We reviewed the literature as to the potential connections between OSCC and bacteria to pinpoint the putative role of distinct bacteria and bacterial symbioses in oral carcinogenesis, and to compare the relevant data with our results.

In a pioneering study, Nagy et al. analysed the biofilm flora present on the surfaces of oral squamous cell carcinomas and on the contiguous healthy mucosa [5]. They found a higher number of both aerobic and anaerobic colony forming bacteria at the tumour sites than at the apparently healthy mucosa. They observed that the frequency of most aerobic species was similar at both sites, except Serratia liquefaciens, Klebsiella pneumoniae, Citrobacter freundii, betahemolyzing streptococci, and Enterococcus faecalis that were isolated more frequently from the biofilm samples obtained from the surfaces of oral carcinomas. Regarding anaerobic species, the frequency of peptostreptococci and lactobacilli was comparable at both sites, whereas Actinomyces spp., Propionibacterium spp., Clostridium spp., Veillonella spp., Fusobacterium spp., Prevotella spp., Porphyromonas spp., and Bacteriodes ureolyticus/gracilis was isolated more frequently from the tumor surface than from the control mucosal surface [5]. In addition, the fungus Candida albicans was detected in a significant fraction of oral carcinomas, but not at control sites [5]. Nagy et al. concluded that the cancer lesion itself may predispose patients with oral carcinoma to both local and systemic infections [5]. In a follow-up study, they demonstrated that topical antimicrobial treatment of oral squamous cell carcinoma lesions effectively reduced the number of biofilm-associated bacteria [42].

In addition to the surface of oral carcinoma, bacteria could be detected inside oral carcinoma samples as well. Using in situ hybridization, Hooper et al. found bacteria throughout the tumor tissue in surface-decontaminated oral squamous cell carcinoma (OSCC) samples [43]. As determined by molecular biological methods, Clavibacter michiganensis, Fusobacterium naviforme, Ralstonia insidiosa and Prevotella spp. were enriched in the tumor-derived samples. In contrast, control tissue samples were enriched in Granulicatella adiacens, Porphyromonas gingivalis, Sphingomonas spp. and Streptococcus mitis/oralis [43]. These 
data suggested that tumor-associated bacteria may play a role in carcinogenesis, and implicated that the acidic and hypoxic microenvironment within neoplasms may favour the growth of distinct bacterial species [43]. Others also raised the point that in addition to Helicobacter pylori, which was recognized as the causative agent of gastric carcinoma and gastric lymphoma in humans, other bacterial species could also play a role in tumorigenesis $[44,45]$. Schmidt et al. analysed swabs taken from oral cancer lesions and clinically normal mucosal surfaces [46]. They observed a reduced abundance of the phyla Firmicutes and Actinobacteria, and an increase in proportion of Fusobacteria at the tumor site, indicating that the altered surface of oral cancer may affect bacterial adherence and select for bacterial populations that elicit inflammatory responses favouring tumor progression [46]. Fusobacterium nucleatum, a Gram negative oral pathogen, was recently implicated in colon carcinogenesis, too [47-49]. Our observation of an increased prevalence of Fusobacterium nucleatum in OPMD samples (see 4.2.3.) is compatible with the data of Schmidt et al. and may indicate that Fusobacterium nucleatum is involved also in the initial stage of oral carcinogenesis. Bebek et al. compared bacterial communities in head and neck cancer (HNCC) and normal mucosa samples [50]. The presence of members of Enterobacteriaceae family and the Tenericutes phylum in HNCC correlated with the hypermethylation of a cellular gene, MDRl coding for multidrug resistance protein 1, a drug efflux pump for xenobiotic compounds [50]. Silencing of MDR1 by hypermethylation was also observed in gastric carcinoma [51]. These data suggested that epigenetic changes induced by bacterial infections may facilitate tumorigenesis [50].

The phylum Tenericutes includes the genera Mycoplasma and Ureaplasma which are prevalent in oral samples from STD patients [52]. Furthermore, Mycoplasma species were detected in rare cases of oral carcinoma and induced malignant cell transformation in vitro [53-56]. However, we did not detect Mycoplasma species in OPMD samples in our study. The composition of salivary microbiota may reflect the microbial diversity of the soft tissues located in the oral cavity. It was suggested that alterations of tumor cell receptors may facilitate the adherence of certain bacteria and the resulting shift of soft tissue microbiota in the oral cavity may affect the levels of bacteria in the saliva $[57,58]$. Thus, in principle, the salivary microbiota could be used as a diagnostic marker of OSCC. 
Carcinogenic metabolites generated by oral bacteria may play a role in carcinogenesis [59]. It was demonstrated that both non-pathogenic Neisseria strains and strains of Streptococcus salivarius and Streptococcus intermedius as well as Corynebacterium spp. and Stomatococcus spp. are capable to convert ethanol to acetaldehyde, a mutagenic and carcinogenic substance [59-61]. Such a mechanism may explain how poor dental status associated with bacterial overgrowth may increase oral cancer risk in patients with tooth loss, poor dentition and inadequate oral hygiene [60, 62]. Homann et al. demonstrated an increase in salivary acetaldehyde production from ethanol in saliva samples of patients with poor dental status [62]. 


\subsection{Indication and technical basis of our experimental setup}

In our experimental setup, we examined the oral microbiome and microbiota of patients diagnosed with OPMD. We compared the microbiome and microbiota of healthy and nonhealthy mucosal surfaces. To gain comparable data, we applied two different methods: the metagenome sequencing of total DNA purified from tissue specimens and MALDI-TOF MS analysis from cultured bacteria. Although Fusobacterium nucleatum was detected by metagenomic sequencing in OPMD biopsies, it could not be detected by MALDI-TOF in OPMD swab samples cultivated in vitro. To resolve this discrepancy, we made qPCR identification of Fusobacterium nucleatum - a bacterium implicated in carcinogenesis [63-68]. Finally, we used an experimental murine model to study mucosal lesions elicited Helicobacter pylori and compared the histopathological alterations observed to the OPMD affected mucosa.

\subsubsection{Metagenome sequencing}

We used ion torrent sequencing, a recently developed method, for metagenome analysis. The Ion Torrent PGM (Personal Genome Machine) was released at the end of 2010. Ion Torrent PGM utilizes a semiconductor sequencing technology, i.e. semiconductor chips. When a nucleotide is incorporated into a DNA molecule by DNA polymerase, a proton is released, an event recorded by the machine. In consecutive cycles the chip is flooded with one nucleotide after another; if there is no incorporation, no signal is recorded [69] For identifying bacterial taxons, the most commonly used method is the sequencing of hypervariable regions in bacterial 16S rRNA genes. 16S rRNA gene sequencing does not require in vitro cultivation of bacteria. Thus, it permits identification of bacterial taxa which do not grow under laboratory circumstances, too. Bacterial 16S ribosomal RNA (rRNA) genes contain nine "hypervariable regions" (V1 - V9) that demonstrate considerable sequence diversity among different bacteria. Most important regions are V1,V2,V3, and V6 [70,71]. 


\subsubsection{MALDI-TOF MS}

In contrast to metagenome sequencing, in vitro cultivation of bacteria is an important step before identification of bacteria by mass spectrometry. Thus, in the case of bacterial or fungal identification with MALDI-TOF MS (Matrix Assisted Laser Desorption Ionization Time Of Flight Mass Spectometry), a microbial colony is analyzed, or in some cases, direct blood culture material, urine, cerebrospinal fluid (CSF), or protein extract is used. Upon drying, the clinical material and the matrix (HCCA, 2,5-DHB, 3- HPA, etc.) cocrystallize and form a solid deposit of sample embedded into the matrix. This sample-matrix crystal, now present on the surface of the metal plate, is irradiated by using a UV laser beam (usually, an $\mathrm{N}_{2}$ laser beam with a wavelength of $337 \mathrm{~nm}$ is utilized in commercial instruments). Specimen with matrix will be together softly ionised to avoid degradation. Proteins within the clinical specimen are analyzed by the mass analyzer to reveal characteristic information about the composition of the sample in the context of a spectrum of mass-to-charge $(\mathrm{m} / \mathrm{z})$ ratios. The $\mathrm{m} / \mathrm{z}$ ratios that are generated and considered when formulating a microbial identification are ribosomal proteins that are unique to their respective bacterial groups or species. In linear TOF, ions generated from the source are accelerated into the flight tube and enter a field-free region where they are separated according to their velocities (and size), before hitting the detector located at the other end of the tube. A reflectron is a focusing element at the end of the TOF instrument that changes the direction of ion travel. A voltage is applied to these lenses and causes a change in the trajectory of that ion. Ions with a higher kinetic energy will penetrate the reflectron deeper than those with a lower kinetic energy [72]. 


\section{Objectives}

The main objective was to investigate the microbiome and microbiota of the OPMD with unknown etiology.

Aims:

1. To analyse and compare the microbiome of the clinically healthy and OPMDaffected oral mucosal tissues by metagenome sequencing technique.

We hypothesized, that the microbiome of clinically healthy and OPMD-affected tissues are different, i.e. pathogenic bacteria are overrepresented in the mucosal samples of OPMD affected tissues.

2. To analyse and compare the microbiota of the healthy and OPMD oral mucosal tissues by MALDI-TOF MS analysis of cultured bacteria.

We hypothesized, that the data obtained by MALDI-TOF MS will be comparable to the results of metagenome sequencing.

3. To verify inflammatory histopathological changes after the mucosal infiltration by H. pylori, a bacterium of known tumorigenic potential, in an in vivo murine experimental model.

We hypothesized, that if OPMDs are caused or triggered by bacteria, the histopathological changes in OPMD lesions may be similar to the changes in H.pylory induced gastric mucosal inflammation and cancer.

4. To compare our results with existing research.

We hypothesized, that the bacterial alterations in OPMD lesions will be comparable those suggested by the current literature for OSCC. 


\section{Materials and Methods}

\subsection{Patient selection}

Potential participants were interviewed by the clinical coordinators at the University of Szeged, Faculty of Dentistry. Every potential participant was informed about the ethical permission of the study and received both written and oral information on the goals and procedures of this study. The initial participants were selected by volunteering activity and the detailed questionnaire on family anamnesis, chronic diseases, medication-, alcohol-, tobaccoand drug consumption, oral hygiene, and sexual habits was filled out by the volunteers. Eligibility was determined based on the results of this questionnaire. Female and male subjects over 18 years of age were eligible for the study, provided that they were able to provide signed and dated informed consent and if they did not meet any of the exclusion criteria. As for the patient group, an established diagnosis of OPMD was also a requirement. Clinical diagnosis was established after clinical examination (inspection, palpation) performed by oral medicine specialist, followed by differentialdiagnostical evaluation of symptoms and anamnestic data. On suspicion of OPMD, volunteers were included into our study and specimens were taken for pathological and microbiological examination (see below). From that examined group we selected patients with the clinical and pathological diagnosis of OLP, and patients with the clinical diagnosis of verrucous leukoplakia supported by pathological diagnosis as well. The exclusion criteria included vital signs outside the acceptable range at the screening visit (i.e., blood pressure $>140 / 90 \mathrm{mmHg}$, oral temperature $>37{ }^{\circ} \mathrm{C}$, heart rate $>100 / \mathrm{min}$ ), pregnancy, the potential subject being a sex worker, topical antibiotic treatment up to 7 days before the screening visit, and the use of the following drugs within 2 months before the screening visit: systemic antibiotics, corticosteroids, cytokines, methotrexate or immunosuppressive cytotoxic agents, or large doses of commercial probiotics ( $\geq 108$ CFU $\mathrm{mL}^{-1}$ organisms per day). No patients were on specific diet, nor on antibiotic therapy in the previous 6 months. The clinical characteristics and brief medical history of the patient group is given in Table 2. The study protocol conformed to the Declaration of Helsinki in all respects and was approved by the Institutional Ethics Committee of the University of Szeged (No. 3161). 
Table 2 Brief medical history of patients

\begin{tabular}{|c|c|c|c|c|c|c|c|}
\hline patient & 1 & 2 & 3 & 4 & 5 & 6 & 7 \\
\hline gender & 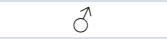 & 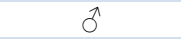 & $+/ 2 \mathrm{PM}$ & $+/ 2 \mathrm{PM}$ & $\sigma^{\pi}$ & 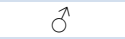 & Q/1 PM \\
\hline age & 60 & 73 & 62 & 57 & 85 & 54 & 77 \\
\hline $\begin{array}{c}\text { clinical } \\
\text { diagnosis }\end{array}$ & $\begin{array}{c}\text { leukoplakia } \\
\text { verrucosa } \\
\text { labii }\end{array}$ & $\begin{array}{c}\text { leukoplakia } \\
\text { proliferativa } \\
\text { verrucosa } \\
\text { gingivae }\end{array}$ & $\begin{array}{l}\text { lichen } \\
\text { reticularis et } \\
\text { atrophicans } \\
\text { buccae et } \\
\text { linguae }\end{array}$ & $\begin{array}{c}\text { lichen } \\
\text { reticularis et } \\
\text { plaque } \\
\text { buccae }\end{array}$ & $\begin{array}{l}\text { lichen } \\
\text { reticularis } \\
\text { buccae }\end{array}$ & $\begin{array}{c}\text { lichen } \\
\text { atrophica } \\
\text { ns } \\
\text { buccae }\end{array}$ & $\begin{array}{c}\text { lichen } \\
\text { reticularis et } \\
\text { atrophicans } \\
\text { buccae et } \\
\text { gingivae }\end{array}$ \\
\hline $\begin{array}{c}\text { pathological } \\
\text { diagnosis }\end{array}$ & $\begin{array}{l}\text { acanthosis } \\
\text { hyper- } \\
\text { parakeratosis } \\
\text { lymphoid } \\
\text { infiltr. } \\
\text { neutrophil } \\
\text { exocytosis } \\
\text { candidosis }\end{array}$ & $\begin{array}{c}\text { epithelial } \\
\text { hyperplasia } \\
\text { hyperkeratosis }\end{array}$ & $\begin{array}{l}\text { chr. lichenoid } \\
\text { inflam. }\end{array}$ & lichen & $\begin{array}{l}\text { chr. lichen } \\
\text { simplex }\end{array}$ & lichen & lichen \\
\hline $\begin{array}{l}\text { systemic } \\
\text { diseases }\end{array}$ & $\begin{array}{c}\text { HT, } \\
\text { onychomyco } \\
\text { sis, MI, cGa, } \\
\text { cDu, IHD }\end{array}$ & $\begin{array}{c}\text { HT, hChol, } \\
\text { hTG }\end{array}$ & no & HT & $\begin{array}{c}\text { HT, } \\
\text { hypothyr, } \\
\text { h.uricemia }\end{array}$ & no & $\begin{array}{l}\text { HT, DM, RA, } \\
\text { h.uricemia, } \\
\text { hyperthyr., } \\
\text { hChol }\end{array}$ \\
\hline allergy & no & no & no & no & $\begin{array}{l}\text { medicine } \\
\text { allergy }\end{array}$ & no & no \\
\hline $\begin{array}{c}\text { years of } \\
\text { smoking }\end{array}$ & 10 & 0 & 0 & 0 & 20 & 20 & 2,5 \\
\hline $\begin{array}{c}\text { alcohol } \\
\text { consumption } \\
\text { /day }\end{array}$ & no & 2-3 unit (wine) & no & no & 2 unit (wine) & no & no \\
\hline $\begin{array}{c}\text { frequency of } \\
\text { tooth- } \\
\text { brushing }\end{array}$ & every week & $\begin{array}{c}\text { many times a } \\
\text { day }\end{array}$ & $\begin{array}{c}\text { many times a } \\
\text { day }\end{array}$ & $\begin{array}{c}\text { many times a } \\
\text { day }\end{array}$ & once a day & $\begin{array}{c}\text { many } \\
\text { times a } \\
\text { day }\end{array}$ & $\begin{array}{c}\text { many times a } \\
\text { day }\end{array}$ \\
\hline $\begin{array}{l}\text { use of oral } \\
\text { rinse }\end{array}$ & no & $\begin{array}{l}\text { many times a } \\
\text { day, changed }\end{array}$ & no & $\begin{array}{l}\text { Listerine/ } \\
\text { Corsodyl }\end{array}$ & no & $\begin{array}{l}\text { Listerine } \\
\text { / Meridol }\end{array}$ & Listerine \\
\hline sexual activity & $\begin{array}{l}\text { none in the } \\
\text { past } 4 \text { yrs }\end{array}$ & active & active & active & active & active & active \\
\hline $\begin{array}{c}\text { oral sexual } \\
\text { activity }\end{array}$ & no & no & no & yes & no & yes & no \\
\hline
\end{tabular}

Supplementary data: no patients were on specific diet, or on antibiotic therapy in the last 6 month. Current medication of patients: 1) Asactal, Concor, Pantoprazol, Roxera, Apranax, Tritace. 2) Lipidil, Xeter, Chinotal, Nortivan, Agen, Aspirin Protect 3)Ø 4) Betaserc, Nootropil, Prenessa, Frontin 5) Tiroxin, Milurit, Warfarin 6) Ø 7) L-Thyroxin, Meloxep, Rosuvastatin, Glucobay, Milurit, Talliton, Frontin, Tramadol, Moxogamma, Metoprolol, Enalapril, Meloxep, Apo-Famotidin, chrom, vitamin-C, vitamin-D, Mg-B6, kurkuma

Abbreviations: PM: postmenopausal; /1/2: parity; HT: hypertension; MI: Myocardial infarct; cGa: chronic gastritis; cDu: chronic duodenitis; IHD: ischemic heart disease; hChol: hypercholesterinaemia; hTG: Hypertrigliceridaemia, DM: diabetes mellitus; RA: rheumatoid arthritis; hypothr: hypothryreosis, hyperthr: hyperthyreosis, h.uricemia: hyperuricemia 


\subsection{Metagenome sequencing}

\subsubsection{Tissue sample collection for metagenome sequencing and histopathological assessment}

As a classical method for tissue sample collection from mucosal lesions in the oral cavity, we used punch biopsy. The biopsy was made with punches $4 \mathrm{~mm}$ in diameter. We collected 2 identical samples from the intralesional area: one sample was taken for hematoxylin-eosin (HE) staining, and an additional sample for metagenome sequencing. Additionally, we also collected a third, control specimen from the ipsilateral healthy mucosa for metagenome sequencing. The specimens contained the mucosa, and submucosal connective tissue. Samples for metagenome sequencing were collected in Eppendorf tubes filled with $20 \mathrm{mM}$ potassium phosphate buffer ( $\mathrm{pH} 7.0)$, and stored at $-20^{\circ} \mathrm{C}$. Tissue samples for HE staining were fixed in formaldehyde solution, and sent for histopathological assessment.

\subsubsection{Metagenomic DNA isolation}

Total DNA was isolated from patient samples as described previously, with minor modifications [73]. Briefly, samples $(600 \mu \mathrm{L})$ were suspended in $650 \mu \mathrm{L}$ of extraction buffer (100 mM TrisCl, pH 8.0, 100 mM EDTA, pH 8.0, 1.5 M NaCl, $100 \mathrm{mM}$ sodium phosphate, $\mathrm{pH} 8.0,1 \% \mathrm{CTAB}) 3.5 \mu \mathrm{L}$ proteinase $\mathrm{K}(20.2 \mathrm{mg} \mathrm{mL}-1)$ and incubated horizontally at $37{ }^{\circ} \mathrm{C}$ for $45 \mathrm{~min}$, next $80 \mu \mathrm{L}$ of $20 \%$ SDS was added and mixed by inversion for several times with further incubation at $60{ }^{\circ} \mathrm{C}$ for $1 \mathrm{~h}$. The sample in each tube was mixed thoroughly every 15 $\min$. The particles were collected by centrifugation $(17,000 \mathrm{~g})$ for $5 \mathrm{~min}$. The supernatant was transferred into clean tubes and was mixed with equal quantity of phenol chloroform and isoamyl alcohol (25:24:1) and extracted three times. DNA was precipitated with 0.7 volume isopropanol, the pellet was washed with $70 \%$ ethanol. Crude DNA pellets were dried and dissolved in $50 \mu \mathrm{L}$ of TE buffer (10 mM Tris- $\mathrm{HCl}, 1 \mathrm{mM}$ sodium EDTA, pH 8.0). Metagenomic DNA was quantified using Qubit ${ }^{\circledR}$ 2.0 Fluorometer. Half of total metagenomic DNA from the healthy and lesion samples were pooled and stored at $-20{ }^{\circ} \mathrm{C}$ for sequencing. 


\subsubsection{Library preparation and sequencing}

Ion Torrent PGM Fragment libraries of $200 \mathrm{nt}$ were generated according to the appropriate protocols (Ion Torrent PGM, Life Tech, USA). $1 \mu \mathrm{g}$ pooled metagenomic DNA from each sample was used for library preparation for each sample. DNA shearing and end-repair was achieved by Ion Xpress ${ }^{\mathrm{TM}}$ Plus Fragment Library Kit, and DNA Purification was carried out by PureLink PCR Purification Kit (Thermo Fisher Scientific, MA). Adapter ligation and nick translation were performed by Ion Shear Plus Reagents Kit (Thermo Fisher Scientific, MA). Size selection was performed in 2\% agarose gel to enrich the 300-350 nt fragments then library amplification was achieved by using Platinum ${ }^{\circledR}$ PCR SuperMix (Thermo Fisher Scientific, MA). ION Library TaqMan qPCR was used for quantitation and Ion Xpress Template 200 ePCR kit was used for the emulsion PCR. Sequencing was performed on Ion Torrent Personal Genome Machine ${ }^{\mathrm{TM}}$ using Ion 318 chip. Ion Torrent Personal Genome Machine $^{\mathrm{TM}}$ sequencing resulted 872,798 sequence reads for sample 1 (control) with an average read length of $219 \pm 71$ bases and 644,200 sequence reads for sample 2 (diseased) with an average read length of $220 \pm 72$ bases.

\subsubsection{Quality assurance}

The MG-RAST software performs a QC (quality control) and an automatic normalization of the FASTQ sequence. For the taxonomical analyses, maximum e-value cut-off of 10-15, minimum percent identity cut-off $90 \%$ and minimum alignment length cut-off 40 nt settings were applied. The overall community composition was determined using the M5nr database. Hits for the eukaryotic data were removed and relative abundance of the bacterial data was calculated. 


\subsection{MALDI-TOF MS analysis}

\subsubsection{Swab sample collection for microbiological culture and identification by MALDI- TOF MS}

We used cotton swabs for collecting bacterial samples. Two individual samples were taken from each patient, one from the surface of the lesion, and another one from the ipsilateral healthy (non-involved) mucosa. The swabs were rolled 4 times over the chosen areas. Then the swabs were placed in anaerobic transport medium (AnaerobeSystems, CA) and sent immediately to the Institute of Clinical Microbiology, University of Szeged, for bacterial cultivation and identification.

\subsubsection{MALDI-TOF MS analysis}

The cells on the cotton swabs in anaerobic transport media were suspended in $1 \mathrm{~mL}$ BrainHeart Infusion (BHI) broth which were diluted in BHI to $10^{-2}$ and $10^{-4}$-fold and selective cultivations were done on chocolate agar, Sabouraud agar for fungi $\left(37^{\circ} \mathrm{C}\right.$ aerobically $\left.24 \mathrm{~h}\right)$, anaerobic Columbia agar (Columbia base supplemented with $2.5 \%$ sheep blood, $1.25 \%$ laked sheep blood, $30 \mathrm{mg} \mathrm{L}^{-1}$ cysteine and $1 \mathrm{mg} \mathrm{L}^{-1}$ Vitamin $\mathrm{K}_{1}$ ), Kanamycin laked Blood agar and Bacteroides Bile Esculin agar $\left(37^{\circ} \mathrm{C}\right.$ anaerobically $\left.48-72 \mathrm{~h}\right)$. The cultivated isolated colonies with different morphologies were recorded and subcultured on Chocolate agar or Anaerobic Columbia agar at $37^{\circ} \mathrm{C}$ for $24 \mathrm{~h}$ in aerobiosis or $48 \mathrm{~h}$ in anaerobiosis, respectively. The cultivated strains were identified to species level by MALDI-TOF MS analysis (microflex, Bruker Daltonics, Bremen, Germany) using the Real Time Classification 3.1 software and database. Species identification was accepted if the $\log$ Scores were $>2$ or genus identification if the $\log$ Scores were between 1.7 and 2 [74]. 


\subsection{Fusobacterium nucleatum qPCR}

\subsubsection{Fusobacterium nucleatum qPCR process}

DNA templates for qPCR were prepared from the undiluted BHI suspensions of the oral swabs (collected for MALDI-TOF MS analysis) by the QiAmp Stool Mini DNA Kit (Qiagen, Germany) as recommended by the supplier. Subsequent quantitative RT-PCRs for Fusobacterium nucleatum were done in StepOne RT-PCR instrument (Invitrogen, CA) using $5 \mu \mathrm{L}$ Brilliant II master mix (Agilent), primers FnucF CTTAGGAATGAGACAGAGATG and FnucR TGATGGTAACATACGAAAGG $0.2 \mu \mathrm{L}$ (35 pmole $/ \mu \mathrm{L})$ each, $1 \mu \mathrm{L}$ of template sample and the following cycling conditions: starting denaturation and hot start activation 95 ${ }^{\circ} \mathrm{C} 10 \mathrm{~min}, 95{ }^{\circ} \mathrm{C} 15 \mathrm{~s}, 56{ }^{\circ} \mathrm{C} 15 \mathrm{~s}$ and $72{ }^{\circ} \mathrm{C} 30 \mathrm{~s}, 40 \times$; and a melting curve from $72{ }^{\circ} \mathrm{C}$ to 95 ${ }^{\circ} \mathrm{C}$ [75] CFUs were calculated comparing the means of threshold cycles to ones of a $F$. nucleatum 10-fold serial dilution samples prepared with the same kit.

\subsubsection{Data analysis}

For the comparison of the numerical data, the Mann-Whitney U test was used in SPSS 21.0 (IBM, NY). 


\subsection{Histological examinations}

\subsubsection{Histopathological assessment of the tissue samples collected from OPMD affected mucosa}

Tissue samples for HE staining were fixed in $4 \%$ buffered formaldehyde, and were sent for histopathological assessment to the Department of Pathology, University of Szeged. HE stained sections were examined with light microscope. Pathological dataset (Table 3) included the absence or presence, together with severity of hyperkeratosis, hyperplasia, reactive atypia, dysplasia, and lichenoid infiltrate [76].

\subsubsection{Examination of mucosal lesions caused by Helicobacter pylori in a murine model}

\subsubsection{Infection}

Specific-pathogen-free six to eight-week-old female Balb/c mice were obtained from Charles River (Germany). Infections with $H$. pylori SS1 strain were established three times over a five-day period by administration via feeding needle of $100 \mu \mathrm{L}$ bacterium suspension in BHIB (109 CFU/mL). Negative control mice remained uninfected. One week later, some animals were sacrificed to verify the success of infection by PCR.

\subsubsection{Histology}

Gastric samples were fixed in $4 \%$ buffered formaldehyde for 24 hours, embedded in paraffin blockers and cut into $4 \mu \mathrm{m}$ serial sections (Leica RM2245). Each sample was autostained (Leica ST5020) by routine Giemsa for highlighting H. pylori bacteria. Slides were archived by virtual microscopy using slide scanner (3DHistech, Budapest, Hungary) [77]. 


\section{Results}

\subsection{Histopathological alterations}

\subsubsection{Histopathological changes in OPMD tissues}

Table 3 The histopathological characteristics of the biopsies

\begin{tabular}{cccccccc}
\hline patient & $\mathbf{1}$ & $\mathbf{2}$ & $\mathbf{3}$ & $\mathbf{4}$ & $\mathbf{5}$ & $\mathbf{6}$ & $\mathbf{7}$ \\
\hline OPMD & + & + & + & + & + & + & + \\
hyperkeratosis & + & ++ & +++ & +++ & + & +++ & +++ \\
hyperplasia & +++ & ++ & + & +++ & - & +++ & +++ \\
reactive atypia & - & + & - & + & - & + & + \\
dysplasia & - & + & - & - & - & - & - \\
lichenoid infiltrate & + & +++ & ++ & +++ & +++ & +++ & ++ \\
MT & - & - & - & - & - & - & - \\
\hline
\end{tabular}

Abbreviations: $(-n o,+$ mild,++ moderate,+++ severe; OPMD: oral potentially malignant disorder; MT: malignant transformation)

Sample selection was based on the histological analysis of oral potentially malignant disorders (OPMDs). Figure 1 shows representative pictures of the OPMD tissue samples with marked hyperkeratosis, acanthosis and lichenoid infiltration. The major histopathological characteristics of the OPMD lesions are summarized in Table 3. Severe hyperkeratosis could be observed in 4 samples, whereas moderate or mild hyperkeratosis was detected in 1 and 2 OPMD biopsies, respectively. The degree of hyperplasia was scored as severe in 4 samples, and it was moderate, mild or absent in 1 sample, each. Reactive atypia was recorded in 4 cases, and it was absent in 3 samples. Dysplasia was observed in a single sample only. Lichenoid infiltrate by lymphoid cells was documented in each biopsy sample (severe: 4 cases; moderate: 2 cases; mild. 1 case). In contrast, there was no sign of malignant transformation in either sample. 


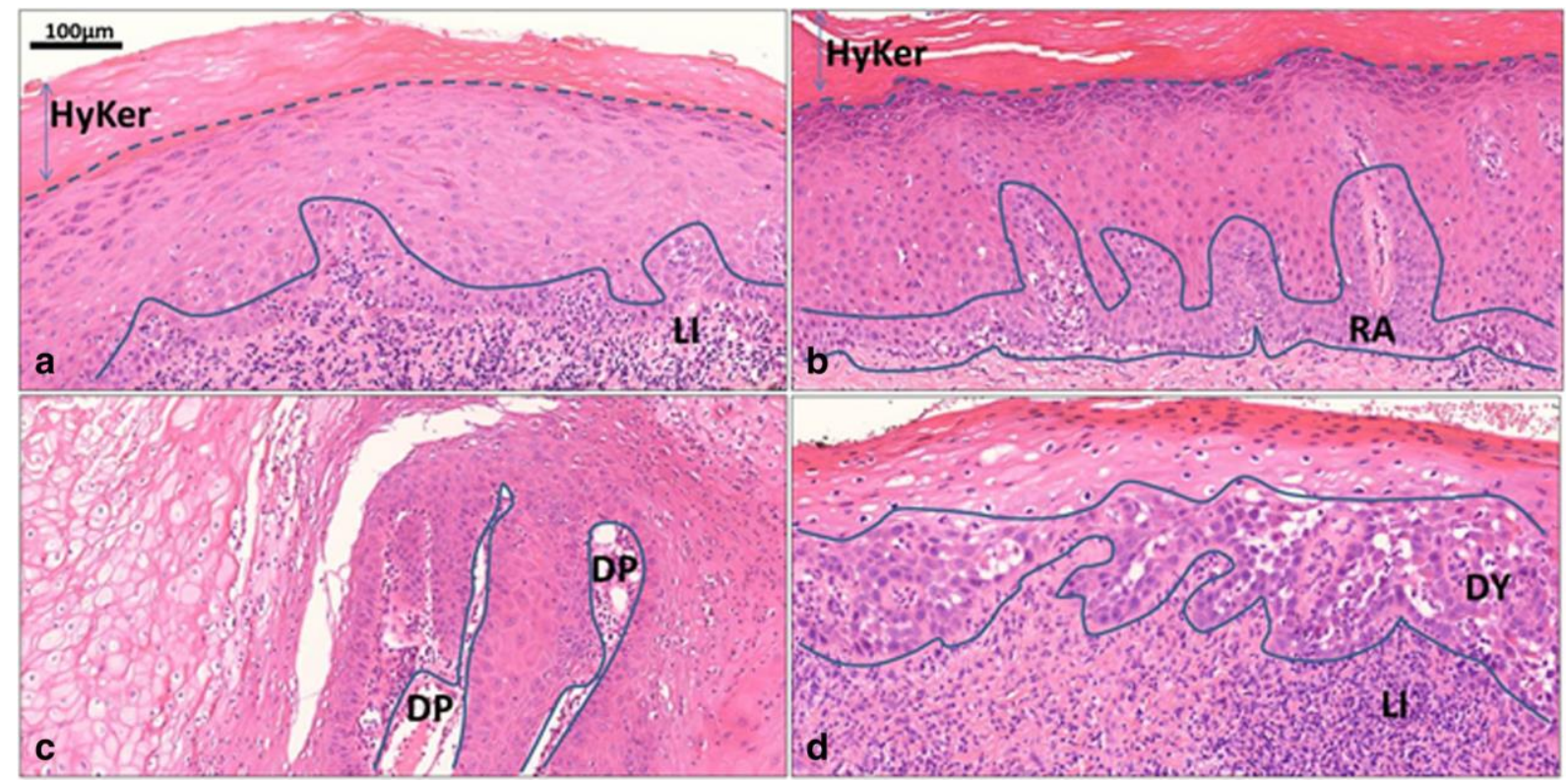

Fig. 1 Representative pictures from OPMD tissue samples with marked hyperorthokeratosis ( $a, b-H y K e r)$, acanthosis and band-like lymho-plasmocytic subepithelial lichenoid infiltrate $(a, d-L I)$. Occasionally there was a marked verrucous hyperplasia with elongated dermal papillae (rete pegs) ( $c-D P)$. Note the regenerative basal cell changes $(b-R A /$ regenerative atypia/), in contrast to mild dysplasia $(d-D Y)$ with focal acantholysis and civatte bodies /HE; OM $112 \times /$

\subsubsection{Histopathological changes in Helicobacter pylori infected gastric mucosa}

Histopathological analyses verified the bacterial colonization and $H$. pylori induced inflammation of gastric mucosa in a murine in vivo experimental model. H. pylori foveolar colonization in gastric fundus and the intra-epithelial mononuclear lymphoid elements of the mucosa shows the infection and the inflammational reaction of the gastric mucosa, but the distribution of lymphocites is different, as in OPMD samples [77]. 


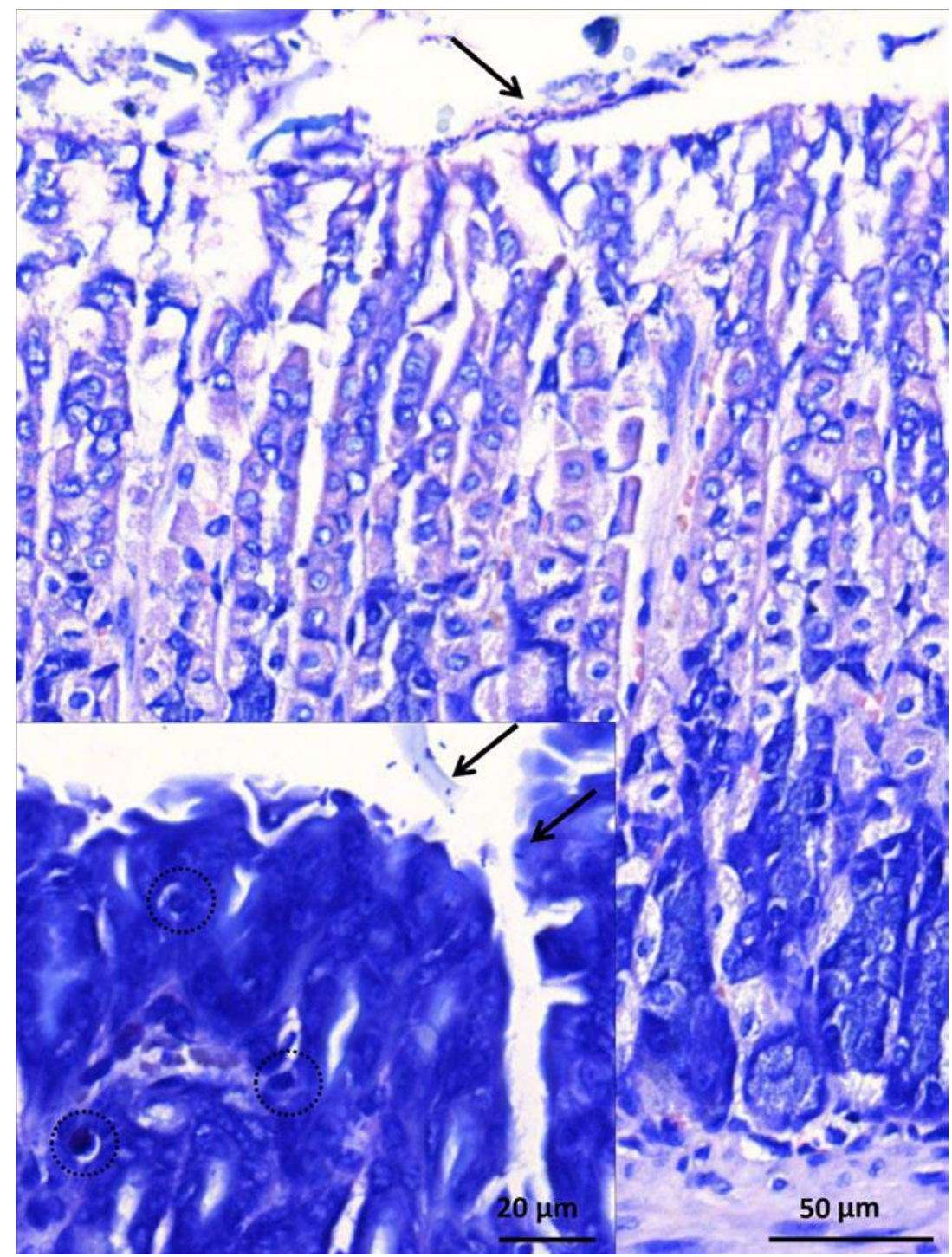

Fig. 2 Long-term H. pylori infection of positive control (infected, untreated) mice. Giemsastained gastric specimens five weeks after infection. Arrows show $H$. pylori foveolar colonization in gastric fundus sample (Giemsa staining; OM 200×), and the intra-epithelial mononuclear lymphoid elements of the mucosa are noted (circles)[77]

We did not detect lichenoid infiltration of lymphoid cells, in contrast to the histological findings described in the case of lichen, or in several cases of verrucous leukoplakias in humans [78]. 


\subsection{Analysis of microbiome/microbiota in biopsy and swab samples from OPMD lesions and healthy oral mucosa}

\subsubsection{Microbiome analysis by metagenome sequencing}

First, we determined the ratio of bacterial DNA in our sample. As punch biopsy samples contain a high amount of human tissue, this step helped us to define the limitation of the study. In healthy oral mucosa samples, $6.78 \%$ of the sequence reads were annotated to the domain Bacteria. The ratio of bacterial sequences was similar $(6.87 \%)$ in the pooled OPMD DNA samples (Fig. 3).
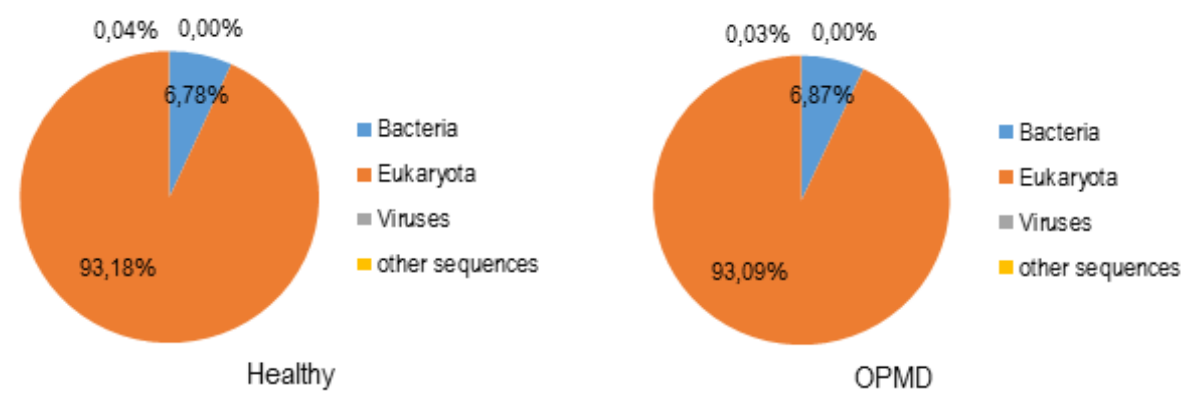

Fig. 3 Distribution of taxonomic domains in OPMD biopsy and healthy oral mucosa samples. There were no significant differences in the distributions of taxonomic domains between the healthy tissue and the OPMD lesion. $6.78 \%$ of bacterial domains were detected in healthy area compared $6.87 \%$ of bacterial domains in white lesions of oral mucosa

Metagenome sequencing revealed that the bacterial diversity in the OPMD biopsies was higher compared to the healthy oral mucosa. Within the Bacteria domain the phyla Firmicutes, Fusobacteria, Proteobacteria, Actinobacteria, Bacteroidetes phyla were present in the healthy oral mucosa. In the OPMD biopsies the same phyla, i.e. Firmicutes, 
Fusobacteria, Proteobacteria, Actinobacteria and Bacteroidetes were identified in descending order of relative abundance; however, two additional phyla, Fibrobacteres and Spirochaetes were observed, too (Fig. 4a). On the species level, 18 bacterial species were detected in the healthy tissue, 35 species were unique for the OPMD lesions and 8 bacterial species were detected both in healthy oral mucosa and the OPMD biopsy samples (Fig. 4b).

a

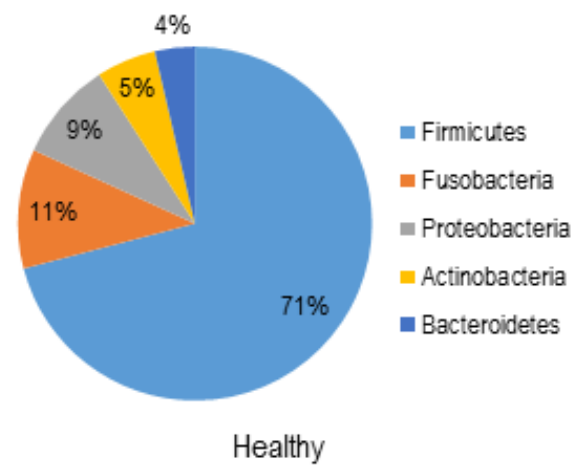

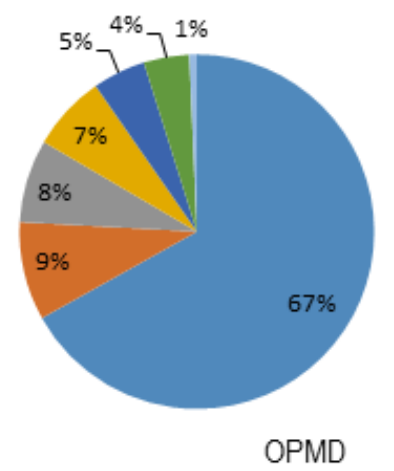

OPMD
- Firmicutes

- Fusobacteria

- Proteobacteria

actinobacteria

- Bacteroidetes

- Fibrobacteres

- Spirochaetes

b

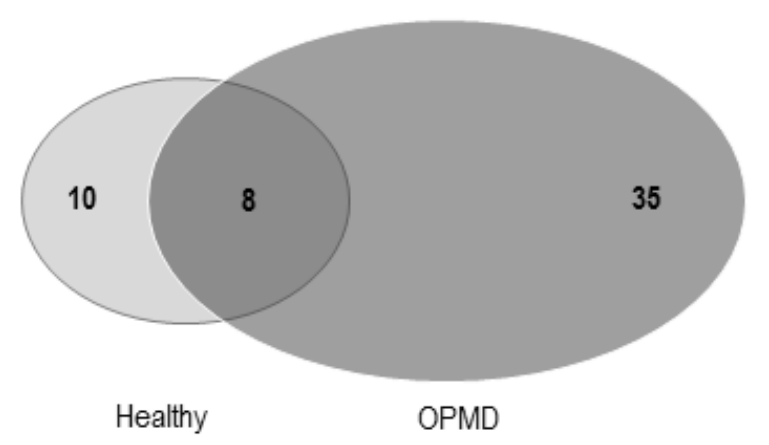

Fig. 4 Bacterial diversity in OPMD biopsy and healthy oral mucosa samples. a Metagenome sequencing revealed that the bacterial diversity in the OPMD biopsies was higher compared to the healthy oral mucosa. Within the Bacteria domain Firmicutes, Fusobacteria, Proteobacteria, Actinobacteria, Bacteroidetes phyla were present in the healthy oral mucosa. In the OPMD biopsies the same phyla were identified in descending order of relative abundance; however, two additional phyla, Fibrobacteres and Spirochaetes were observed, too. $\boldsymbol{b}$ Metagenome sequencing detected 18 different bacterial species in healthy tissue and 43 species in the OPMD lesion, whereas 8 bacterial species were detected in both samples 
Analysis of bacterial species by metagenome sequencing showed a marked, 2.38 fold increase in bacterial diversity in the OPMD tissue samples compared to healthy mucosa (Fig. 5, Table $4)$.

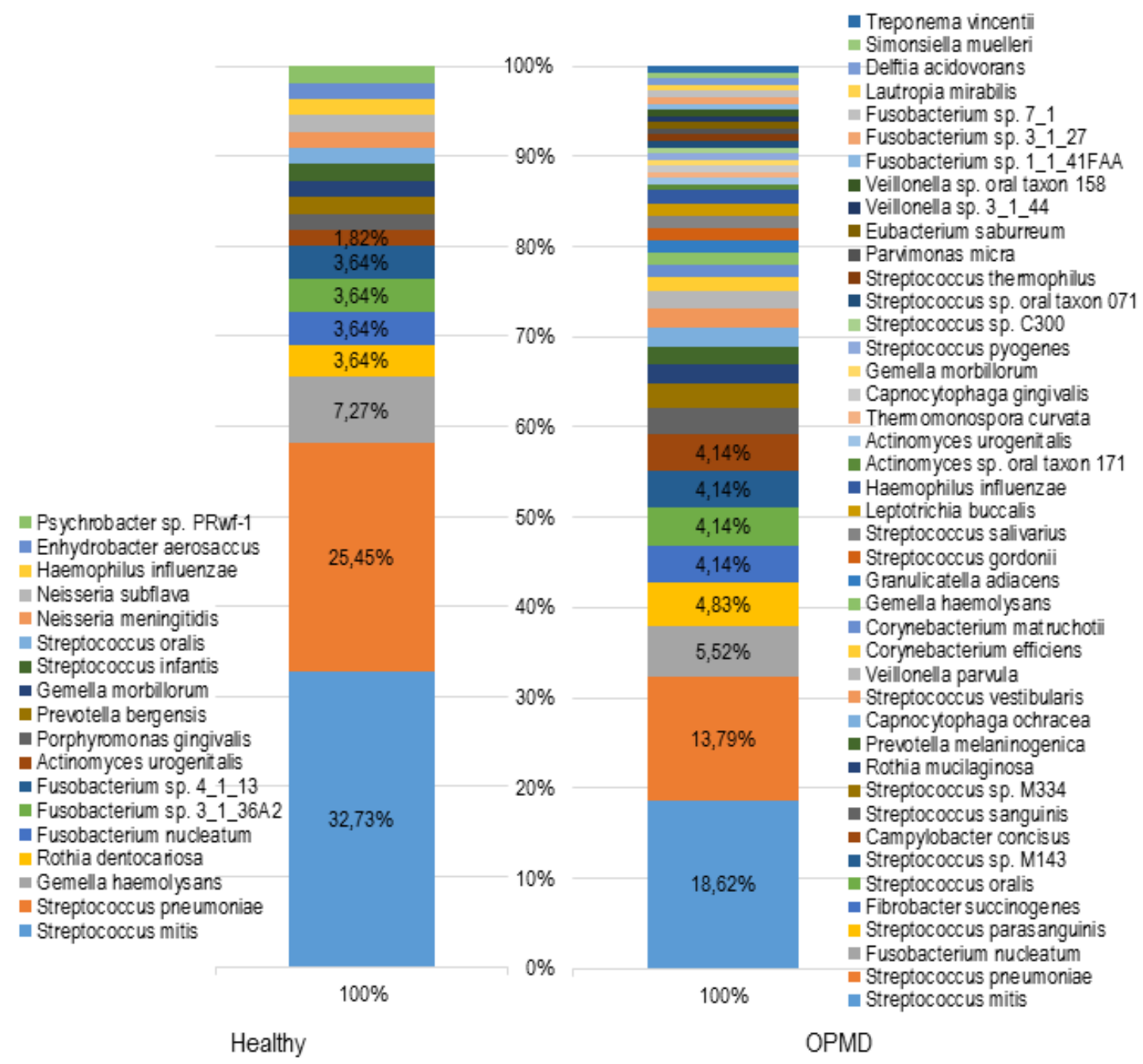

Fig.5 Comparison of the detected bacterial species Metagenome sequencing detected 18 different bacterial species in healthy tissue and 43 species in the OPMD lesion

Based on the data of metagenome sequencing, the relative abundance of Streptococcus sp. did not show a significant difference between the healthy tissue and the OPMD lesions: it was 
$61.82 \%$ in healthy tissue and 58.62\% in OPMD, respectively (Fig. 6a) However, the relative abundance of Streptococcus mitis was found to be dramatically decreased in OPMD: it was only $18.62 \%$, compared to $32.73 \%$ in the healthy tissue (Fig. 6b). Moreover, examining all Streptococcus species their prevalence in the OPMD lesions was found to be more diverse compared to the healthy tissue (Table 4).

a
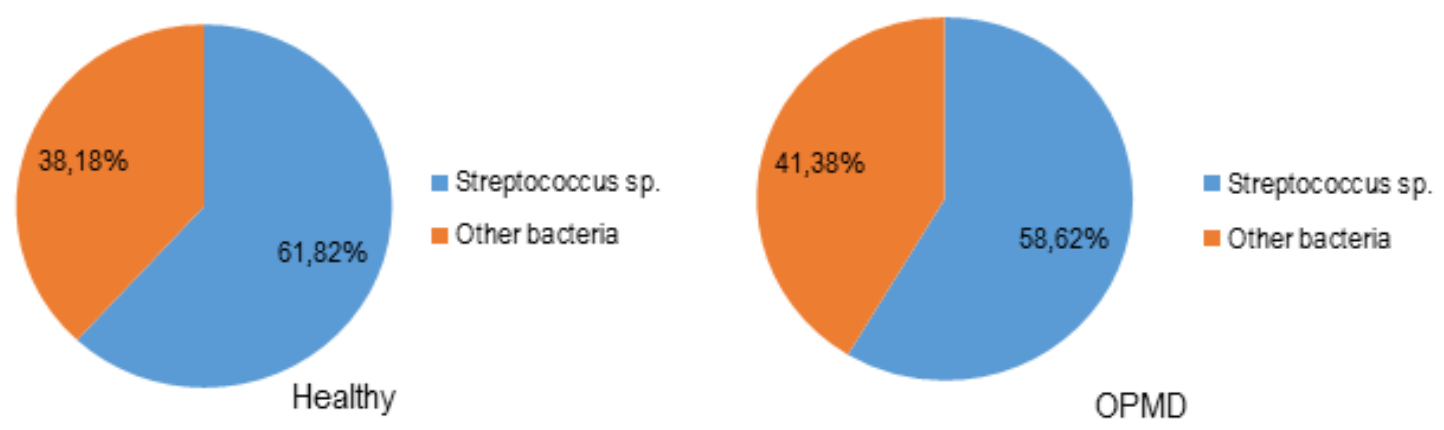

b
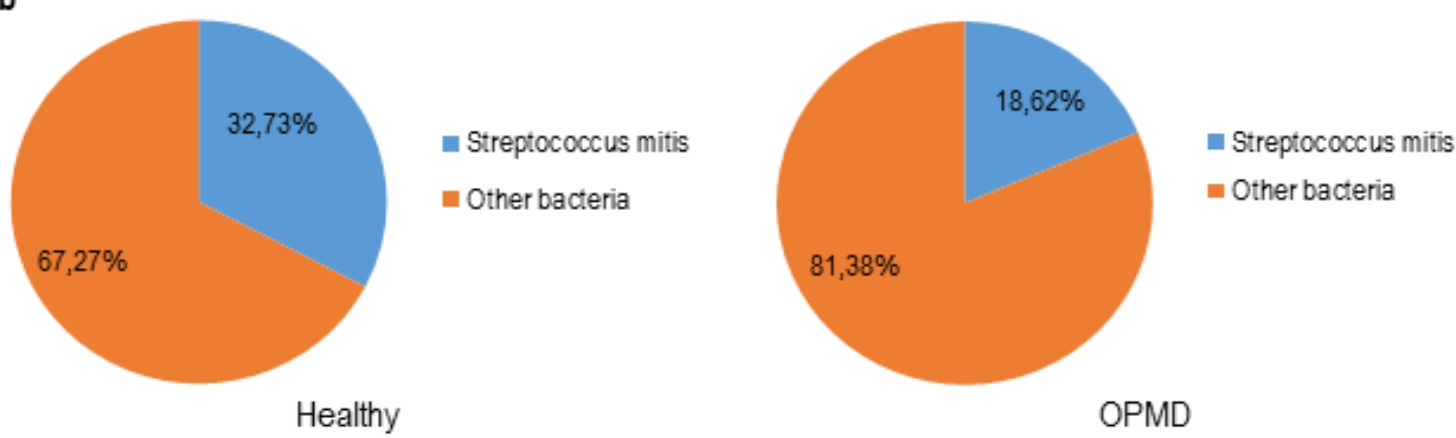

Fig. 6 Relative abundance of Streptococci in the mucosal lesion

a The ratio of Streptococci to all oral bacteria was not significantly different in the healthy tissue compared to the OPMD lesion.

$\boldsymbol{b}$ The relative abundance of Streptococcus mitis dramatically decreased in OPMD, as compared to the healthy tissue 
Table 4 Comparison of bacterial diversity in the healthy mucosa and the OPMD lesion

\begin{tabular}{|c|c|c|c|}
\hline Species & Phylum & Healthy & OPMD \\
\hline Streptococcus mitis & Firmicutes & $32,73 \%$ & $18,62 \%$ \\
\hline Streptococcus pneumoniae & Firmicutes & $25,45 \%$ & $13,79 \%$ \\
\hline Gemella haemolysans & Firmicutes & $7,27 \%$ & $1,38 \%$ \\
\hline Rothia dentocariosa & Actinobacteria & $3,64 \%$ & 0,021 \\
\hline Fusobacterium nucleatum & Fusobacteria & $3,64 \%$ & $5,52 \%$ \\
\hline Actinomyces urogenitalis & Actinobacteria & $1,82 \%$ & $0,69 \%$ \\
\hline Streptococcus oralis & Firmicutes & $1,82 \%$ & $4,14 \%$ \\
\hline Haemophilus influenza & Proteobacteria & $1,82 \%$ & $1,38 \%$ \\
\hline Fusobacterium sp. 3_1_36A2 & Fusobacteria & $3,64 \%$ & 0 \\
\hline Fusobacterium sp.4_1_13 & Fusobacteria & $3,64 \%$ & 0 \\
\hline Porphyromonas gingivalis & Bacteroidetes & $1,82 \%$ & 0 \\
\hline Prevotella bergensis & Bacteroidetes & $1,82 \%$ & 0 \\
\hline Gemella morbillorum & Firmicutes & $1,82 \%$ & 0 \\
\hline Streptococcus infantis & Firmicutes & $1,82 \%$ & 0 \\
\hline Neisseria meningitides & Proteobacteria & $1,82 \%$ & 0 \\
\hline Neisseria subflava & Proteobacteria & $1,82 \%$ & 0 \\
\hline Enhydrobacter aerosaccus & Proteobacteria & $1,82 \%$ & 0 \\
\hline Psychrobacter sp. PRwf-1 & Proteobacteria & $1,82 \%$ & 0 \\
\hline Streptococcus parasanguinis & Firmicutes & 0 & $4,83 \%$ \\
\hline Fibrobacter succinogenes & Fibrobacteres & 0 & $4,14 \%$ \\
\hline Streptococcus sp. M143 & Firmicutes & 0 & $4,14 \%$ \\
\hline Campylobacter concisus & Proteobacteria & 0 & $4,14 \%$ \\
\hline Streptococcus sanguinis & Firmicutes & 0 & $2,76 \%$ \\
\hline Streptococcus sp. M334 & Firmicutes & 0 & $2,76 \%$ \\
\hline Prevotella melaninogenica & Bacteroidetes & 0 & $2,07 \%$ \\
\hline Capnocytophaga ochracea & Bacteroidetes & 0 & $2,07 \%$ \\
\hline Streptococcus vestibularis & Firmicutes & 0 & $2,07 \%$ \\
\hline Veillonella parvula & Firmicutes & 0 & $2,07 \%$ \\
\hline Rothia mucilaginosa & Actinobacteria & 0 & $2,07 \%$ \\
\hline Corynebacterium efficiens & Actinobacteria & 0 & $1,38 \%$ \\
\hline Corynebacterium matruchotii & Actinobacteria & 0 & $1,38 \%$ \\
\hline Granulicatella adiacens & Firmicutes & 0 & $1,38 \%$ \\
\hline Streptococcus gordonii & Firmicutes & 0 & $1,38 \%$ \\
\hline Streptococcus salivarius & Firmicutes & 0 & $1,38 \%$ \\
\hline Leptotrichia buccalis & Fusobacteria & 0 & $1,38 \%$ \\
\hline Actinomyces sp. oral taxon 171 & Actinobacteria & 0 & $0,69 \%$ \\
\hline Thermomonospora curvata & Actinobacteria & 0 & $0,69 \%$ \\
\hline Capnocytophaga gingivalis & Bacteroidetes & 0 & $0,69 \%$ \\
\hline Gemella morbillorum & Firmicutes & 0 & $0,69 \%$ \\
\hline Streptococcus pyogenes & Firmicutes & 0 & $0,69 \%$ \\
\hline Streptococcus sp. C300 & Firmicutes & 0 & $0,69 \%$ \\
\hline Streptococcus sp. oral taxon 071 & Firmicutes & 0 & $0,69 \%$ \\
\hline Streptococcus thermophiles & Firmicutes & 0 & $0,69 \%$ \\
\hline Parvimonas micra & Firmicutes & 0 & $0,69 \%$ \\
\hline Eubacterium saburreum & Firmicutes & 0 & $0,69 \%$ \\
\hline Veillonella sp.3_1_44 & Firmicutes & 0 & $0,69 \%$ \\
\hline Veillonella sp. oral taxon 158 & Firmicutes & 0 & $0,69 \%$ \\
\hline Fusobacterium sp.1_1_41FAA & Fusobacteria & 0 & $0,69 \%$ \\
\hline Fusobacterium sp. 3_1_27 & Fusobacteria & 0 & $0,69 \%$ \\
\hline Fusobacterium sp.7_l & Fusobacteria & 0 & $0,69 \%$ \\
\hline Lautropia mirabilis & Proteobacteria & 0 & $0,69 \%$ \\
\hline Delftia acidovorans & Proteobacteria & 0 & $0,69 \%$ \\
\hline Simonsiella muelleri & Bacteroidetes & 0 & $0,69 \%$ \\
\hline Treponema vincentii & Spirochaetes & 0 & $0,69 \%$ \\
\hline
\end{tabular}


According to the results of metagenome sequencing, the relative abundance of Fusobacterium nucleatum was higher in the OPMD lesions $(5.52 \%)$, compared to the healthy tissue $(3.64 \%)$ (Fig. 7).
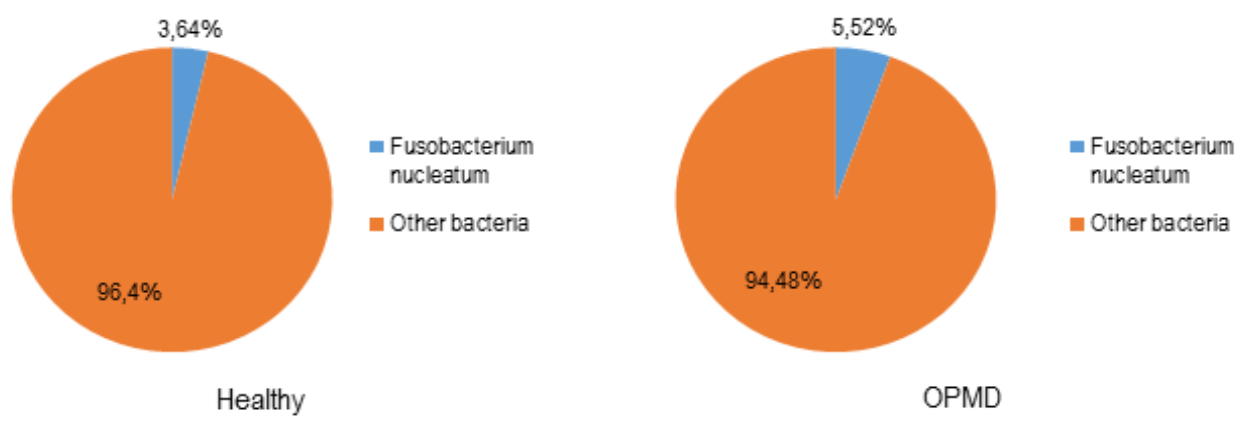

Fig. 7 Fusobacterium nucleatum in OPMD and healthy oral mucosa biopsies

The ratio of Fusobacterium nucleatum was markedly increased in the OPMD lesions, as compared to the healthy tissue detected by metagenome sequencing

In OPMD, the relative abundance of Fusobacterium nucleatum and Streptococcus oralis was $5.52 \%$ and $4.14 \%$, respectively. In healthy mucosa, the corresponding data were lower $(3.64 \%$ versus $1.82 \%)$. The abundances of the species Gemella haemolysans (1.38\%), and the above mentioned S.mitis (18.62\%) decreased markedly in OPMD as compared to the healthy tissue (7.27\% and $32.73 \%$ ) (Table 4). There where 35 different bacterial species detected in the OPMD lesions which were not represented in the healthy tissue, e.g., Streptococcus parasanguinis, Fibrobacter succinogenes, Campylobacter concisus, Streptococcus sanguinis, Rothia mucilaginosa, Prevotella melaninogenica, Capnocytophaga ochracea, Streptococcus vestibularis, Veillonella parvula, Corynebacterium efficiens, Corynebacterium matruchotii, Granulicatella adiacens, Streptococcus gordonii, Streptococcus salivarius, Leptotrichia buccalis, Thermomonospora curvata, Capnocytophaga gingivalis, Streptococcus pyogenes, Streptococcus thermophiles, Parvimonas micra, Eubacterium saburreum, Lautropia mirabilis, Delftia acidovorans, Simonsiella muelleri, Treponema vincentii, etc., in descending order of the abundance. 


\subsubsection{Analysis of microbiota by MALDI-TOF MS}

Compared to metagenome sequencing data, the MALDI-TOF MS based method indicated markedly different results. We detected 41 bacterial species in healthy tissue and 36 species in the OPMD lesions (Fig. 8). There were 25 overlapping bacterial species in healthy tissue samples and OPMD lesion. Although the MALDI-TOF MS results showed a similar decreasing- tendency regarding the presence of Streptococcus species in OPMD lesions, we did not find the expected markedly increasing tendency as to the presence of Fusobacterium nucleatum.

These results highlight the limitations of metagenome sequencing and MALDI-TOF MS methods. While metagenome sequencing is representative of the information content of all DNA present in the sample, MALDI-TOF MS only provides information on the presence of bacteria that grow well under the given culture conditions.

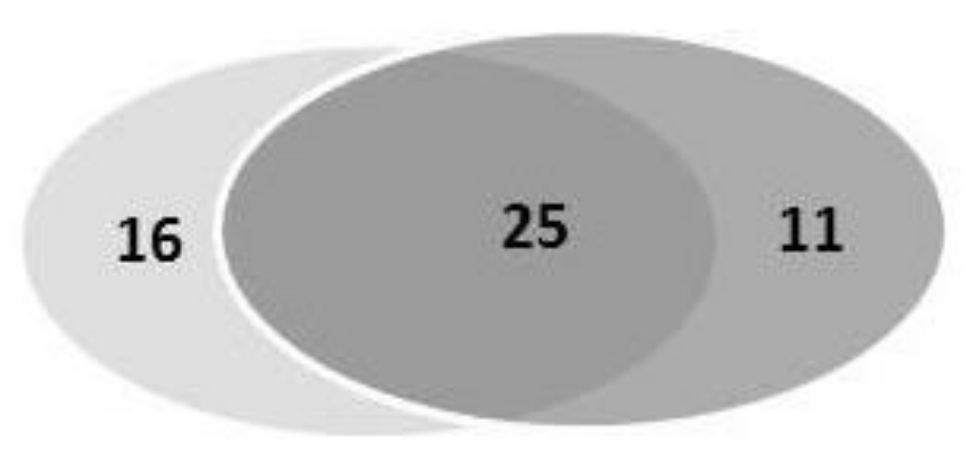

Healthy

OPMD

Fig. 8 MALDI-TOF MS analysis detected 41 different bacterial species in healthy tissue and 36 species in the lesion. 25 bacterial species were detected in both samples 


\subsubsection{Quantitation of Fusobacterium nucleatum in oral swab samples by qPCR}

As F. nucleatum was implicated in carcinogenesis in several cancers [63-67], we verified the results of metagenome sequencing and MALDI-TOF MS by F. nucleatum-specific qPCR. As Fig. 9 shows, the number of bacteria (CFUs) was significantly higher $(\mathrm{P}<0.0001)$ in oral swab samples taken from the surface of OPMD lesions than in those from the healthy mucosa. This difference is remarkable, even when one considers the high inter-individual variability of the samples.

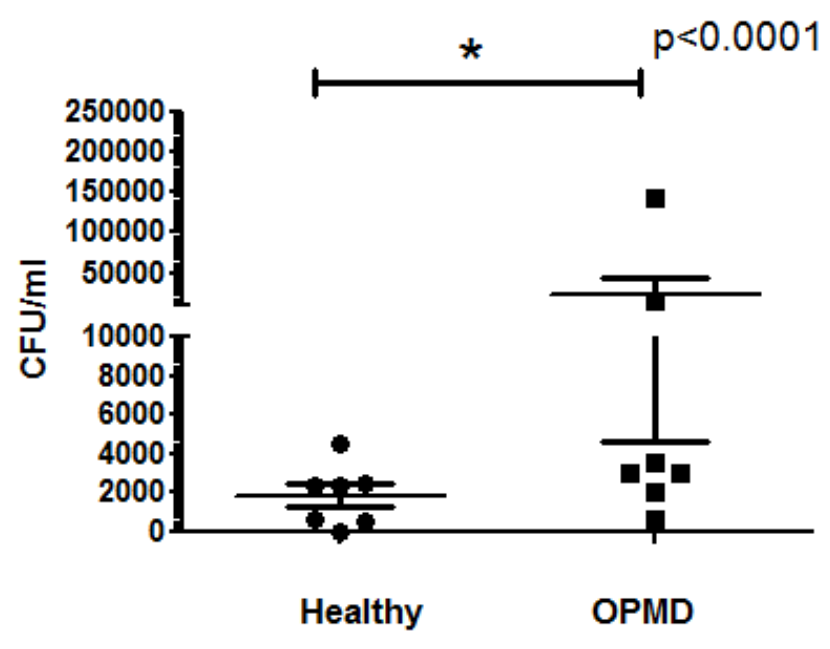

Fig. 9 Fusobacterium nucleatum-specific qPCR of healthy oral mucosa and the OPMD samples. In vitro bacterial cultures of oral swab samples fromhealthy mucosa and OPMD lesions were assayed using Fusobacterium nucleatum-specific qPCR. Colony forming units (CFUs) were calculated by comparing the means of thresold cycles to those of serial dilution samples of control F. nucleatum cultures.

OPMD showed a significantly higher colonization by F. nucleatum compared to healthy mucosa 


\section{Discussion}

It was suggested that OPMD (oral potentially malignant disorders) and oral cancer frequently associated with alcohol and tobacco consumption, and affected mainly males in their fifties [2]. Other epidemiological data indicated, however, that a distinct subgroup of oral cancer patients aged 45 years and under showed no significant exposure to the traditional risk factors tobacco and alcohol [4]. Accordingly, we included a wide range of patients in our study, without strict limitations on age or sex. Because bacteria were implicated in oral carcinogenesis (reviewed by Hettmann et al., 2016 [13]), we compared the microbiome and microbiota of healthy mucosa and OPMD tissues of oral cavity.

Regarding the putative role of distinct oral bacteria in tumorigenesis, the association of Fusobacterium nucleatum with colorectal carcinoma and oral carcinoma was documented [67, 68]. Moreover, it was suggested that a distinct subspecies of $F$. nucleatum, such as $F$. nucleatum subsp. polymorphum may play an etiopathogenetic role in oral carcinogenesis [65].

A study also described the presence of $F$. nucleatum in desquamative gingivitis [79]. Our finding that $F$. nucleatum is present in OPMD may be exploited to develop a novel therapeutic strategy of distinct oral disorders such as oral lichen planus. In addition, one may speculate that a targeted antibiotic therapy could be beneficial in preventing the development of oral cancer in a subset of OPMD patients.

We observed that the relative abundance of Streptococcus mitis decreased dramatically in the pathological niche of OPMD. Since Streptococcus species are characteristic components of the oral flora, the quantitative analysis of these bacteria is indispensable for the understanding of pathological processes. Streptococci comprise of a wide variety of bacterial species that interact with other members of the oral microbiome. It was suggested that $S$. mitis is involved in the maintenance of a healthy oral flora by affecting adhesion and biofilm formation by periodontal pathogens to [80-82].

Therefore, it is an interesting and important finding that the relative abundance of $S$. mitis in the OPMD lesions decreased to nearly the half of the healthy area. This observation may possibly be exploited for therapeutic purposes: similarly to the reconditioning of vaginal 
Lactobacillus balance [83], the restitution of $S$. mitis niche in OPMD could also have beneficial effects [84].

In comparison with earlier results [13], Actinomyces spp., Veilonella spp., Fusobacterium spp., Prevotella spp., Streptococcus spp., and Corynebacterium spp. were represented in our OPMD samples, similarly to OSCC with mentioned putative role in carcinogenesis. Gemella spp. as a suggested spp with putative role in OSCC carcinogenesis was found also in our OPMD samples, but was represented in higher rate in healthy mucosa. We did not find in our OPMD samples bacteria characteristic for OSCC, such as Serratia liquefaciens, Klebsiella pneumoniae, Citrobacter freundii, Enterococcus faecalis, Propionibacterium spp., Clostridium spp., Porphyromonas spp., Bacteroides ureolyticus/gracilis, Clavibacter michigenensis, Ralstonia insidosa, Peptostreptococcus stomatis, Johnsonella ignova, Enterobacteriaceae (family), Tenericutes (phylum), Stomatoccus spp.

Similarly to metagenome sequencing, a moderate change of normal flora bacteria Streptococci was detected also with MALDI-TOF MS. However, in contrast to the sequencing data, overrepresentation of Fusobacterium nucleatum was not detected in the OPMD samples with MALDI-TOF MS. Detection of Fusobacterium nucleatum by qPCR in the very same samples that were used for MALDI-TOF analysis revealed that the sensitivity of culturing followed by MALDI-TOF MS examination for $F$. nucleatum was in our case low. The results obtained from the different methods used indicated that metagenome sequencing yielded a more diverse bacterial community in OPMD than MALDI-TOF MS. It should not be forgotten that while metagenome sequencing allows the identification almost all represented DNA sequences, MALDI-TOF MS builds on the classic methodology of microbiological culturing since MALDI-TOF MS uses samples which were cultured from the oral swabs. One may also argue that swab samples used for culturing were taken from the surface of the mucosa of OPMD lesions. Thus, they did not represent the microbiota of deeper mucosal layers. Perhaps, in a follow-up study, it would be more efficient to use brush biopsy to sample altered mucosal surfaces for culturing before MALDI-TOF MS. Differences between results of metagenome sequencing and MALDI-TOF MS analysis reveals, we should assess the limitations of different methods to choose the appropriate experimental setup in follow-up study. 
Taken together, we presented evidence for the alteration of microbiome and microbiota of OPMD patients. We detected an increased bacterial diversity by metagenome sequencing in the OPMD lesions compared to the healthy oral mucosa. In addition, decreased relative abundance of $S$. mitis and an increased relative abundance of $F$. nucleatum may play a role in the transition of OPMDs to oral cancer.

If there is an association between a bacterial species and oral carcinogenesis, it is important to know, what kind of mechanism leads to malignant transformation. One possibility is that epigenetic alterations facilitate the development of OPMDs or induce dysplastic changes [50, 51]. Production of mutagenic metabolites may also result in genotypic and phenotypic alterations [21, 59-62]. In addition, direct effect of bacterial antigenes on cell surfaces [85, 86], intracellular invasion of bacteria in mucosal cells [21, 87] or the effect of mucosal inflammation caused by immunresponse of immune cells against bacteria $[88,89]$ may also contribute to tumorigenesis. Histopathologically, we can examine the presence of mucosal histologycal and cellular alterations, accumulation of inflammatory cells and bacterial invasion.

In our experimental setup we examined lichen planus (OLP) and proliferative verrucous leukoplakia (PVL), two etiologically different diseases. In case of OLP, there are conflicting data regarding the role of bacterial infection. [30-37] In our clinical experience the level and duration of CD8+ T cell mediated lichenoid ,interface type” autoimmun inflammation is in correlation with the development of dysplastic changes [90-92]. In case of OLP it is also known that if the mucosal surface of OLP is infected with Candida albicans, some pathogen bacteria or viruses, the inflammation is usually more severe, and the lichen tends to be atrophic, erosive, or ulcerative clinical type. After the eradication of pathogens, the inflammation and clinical type of OLP will be again milder [93, 94].

To compare the histopathological inflammational signs of our OPMD tissue samples, we checked a known mucosal infection wich increase the risk of malignant transformation (Fig. 2) - inflamed gastric mucosa of Helicobacter pylori infected mice [95]. In the murine model, there were numerous of mononuclear lymphoid elements in the inflamed mucosa. However we could not find lichenoid infiltration of lymphoid cells described in case of lichen, or in several cases of verrucous leukoplakias [78]. 
In OPMD cases, some inducing or initiator factor is needed for transition to the cancerous malformation. This factor can also be an inflammatory process. The role of $H$. pylori in various tumorous degeneration as well as in OSCC has been demonstrated [96, 97]. In OPMD there are both supporting as well as negative evidences for a connection with $H$. pylori infection [20, 31-35]. In our animal model we detected the signs of inflammation in $\boldsymbol{H}$. pylori infected gastric mucosa. Based on the above, although the limitations of the model should be taken into consideration, one can not exclude that $H$. pylori infection or inflammation elicited by other oral bacteria could possibly contribute to the transition of OPMD to carcinoma. Generally, microbial induced chronic inflammation may play a role in development and malignant transformation of OPMD. 


\section{Conclusion}

Although our work is not suitable to answer the „Chicken or the Egg” problem - that the suggested microbial changes are cause or effect of OPMD - in all aspects, but we have found that the bacterial colonization of mucosa has already altered in OPMD tissues. Some alterations correlate with results of the studies about the putative role of bacteria in OSCC development. Our most conspicouos finding was that Fusobacterium nucleatum is overrepresented in OPMD tissue samples. Fusobacterium nucleatum was implicated in malignant transformation of mucosal cells of gastrointestinal tract, especially in the colon, and several studies suggest a similar role for $F$. nucleatum and other pathogenic bacteria in oral mucosal cells [63-68].

1. We found that the microbiome of clinically healthy and OPMD affected oral tissues are different: pathogenic bacteria were overrepresented in the mucosal samples of OPMD affected tissues. Our results showed that Fusobacterium nucleatum, a bacterium implicated in tumorigenesis, is overrepresented in the samples from OPMD affected regions. In addition, we observed an increased bacterial diversity in OPMD, compared to the microbiota of healthy oral mucosa. These observations may form the basis of novel therapeutic approaches preventing oral carcinogenesis in a subset of patients with OPMD.

2. The comparison of MALDI-TOF MS and metagenome sequencing provided partially overlapping results.

3. We found inflammatory signs both in human OPMD samples and in an in vivo murine model of $H$. pylory-infected gastric mucosa. This may indicate that one can't exclude a potential role for $H$. pylori infection or inflammation elicited by other oral bacteria could play a role in the transition of OPMD to carcinoma in humans. 
4. Comparison of the microbiome of OPMD samples analysed in our study and the microbiome/microbiota of OSCC lesions reviewed in the literature revealed that a wide variety of non-overlapping sets of bacterial taxa were found to be associated with OSCC lesions. Fusobacterium nucleatum emerged from the data as the best potential candidate contributing to te transition of OPMD to OSCC.

\section{Summary of accomplished objectives}

1. Pathogenic bacteria are overrepresented in the mucosal samples of OPMD affected tissues.

2. Specificity and sensitivity of metagenome sequencing and cultivation based MALDITOF MS is markedly different to oral bacteria.

3. Helicobacter pylori caused gastric inflammation in mice could be an experimental model of bacterial inflammation triggered carcinogenesis of oral mucosal surfaces. (Keeping the limitations caused by differences between subjects and modellorganisms in mind.)

4. Fusobacterium nucleatum emerged from the data as the best potential candidate contributing to the transition of OPMD to OSCC. 


\section{Acknowledgements}

I wish to thank my co-supervisor, Professor Katalin Nagy, Former Dean of the Faculty of Dentistry, Head of Oral Surgery Department for her support and supervision.

Also I would like to thank my co-supervisor, Krisztina Buzás, Ph.D., who made it possible for me to conduct research at the faculty while working as a clinician, helped in theoretical questions and in organisation of laboratory works.

I'm also grateful to Professor István Sonkodi who helped me with patient selection and kindly allowed me to work with some of his patients. He ground me in clinical and theoretical aspects of oral medicine.

I would like to thank the research team Edina Gyukity-Sebestyén, Mária Harmati, Gabriella Dobra. They put me up to the ropes about all the lab skills and work with animal models.

I'm also grateful to Professor János Minárovits. He showed me a way into the fantastic world of research.

I thank István Németh, Ph.D. for his cooperation in the histological investigations.

I would like to thank Maróti Gergely Ph.D., for his cooperation in metagenome sequencing.

I thank Edit Urbán, Ph.D., József Sóki, Ph.D. and Viktor Fenyvesi ${ }^{\dagger}$ for their cooperation in MALDI-TOF MS and PCR examination.

And also thank to Gábor Braunitzer, Ph.D. who showed me the stylish way of publishing.

Last but not least I would like to thank to my family.

The Ph.D. thesis was supported by OTKA NKFI-6-K 11493, GINOP-2.3.2-15-2016-00011, GINOP-2.3.2-15-2016-00015 projects and János Bolyai Research Fellowship. 


\section{References}

1. Ferlay J, Soerjomataram I, Ervik M, Dikshit R, Dikshit R, Eser S, Mathers C, et al (2013) GLOBOCAN 2012 v1.0, Cancer Incidence and Mortality Worldwide: IARC CancerBase No. 11. Lyon, France: International Agency for Research on Cancer http://globocan.iarc.fr.

2. Morse DE, Psoter WJ, Cleveland D et al (2007) Smoking and drinking in relation to oral cancer and oral epithelial dysplasia. Cancer Causes Control 18(9):919-929

3. Jepsen SA, Closmann JJ (2008) The insidious nature and presentation of oral squamous cell carcinoma in the low-risk population. Gen Dent 56(1):78-82

4. Llewellyn CD, Linklater K, Bell J et al (2003) Squamous cell carcinoma of the oral cavity in patients aged 45 years and under: a descriptive analysis of 116 cases diagnosed in the South East of England from 1990 to 1997. Oral Oncol 39(2):106-114

5. Nagy KN, Sonkodi I, Szöke I et al (1998) The microflora associated with human oral carcinomas. Oral Oncol 34(4):304-308

6. Sarode SC, Sarode GS, Tupkari JV (2012) Oral potentially malignant disorders: precising the definition. Oral Oncol 48(9):759-760

7. van der Waal I (2009) Potentially malignant disorders of the oral and oropharyngeal mucosa; terminology, classification and present concepts of management. Oral Oncol 45(4$5): 317-323$

8. Decsi G, Tarnai Z, Zsedényi A, Buzás K, Nagy K, Sonkodi I, Minárovits J (2014) A szájüregi laphámrák és praeblastomatosisai: a papillomavírus-fertőzés szerepe és a korai diagnózis lehetőségei = Oral carcinoma and its preblastomatoses: The role of papillomavirus infection and the prospects of early diagnosis. Lege Artis Medicinae 24(3):111-119

9. Sonkodi I (2009) Oral and Maxilofacial medicine. Semmelweis Publisher, Budapest

10. Silverman S Jr, Gorsky M (1997) Proliferative verrucous leukoplakia. A follow-up study of 54 cases. Oral Surg Oral Med Oral Pathol Oral Radiol Endod 84(2):154-157

11. Fehér E, Gáll T, Murvai M et al (2009) Investigation of the occurrence of torque tenovirus in malignant and potentially malignant disorders associated with human papillomavirus. $\mathrm{J}$ Med Virol 81(11):1975-1981

12. Fehér E, Kardos G, Gáll T et al (2011) Comparison of diversity of torque teno virus 1 in different mucosal tissues and disorders. Acta Microbiol Immunol Hung 58(4):319-337

13. Hettmann A, Demcsák A, Decsi G, Bach Á, Pálinkó D, Rovó L, Nagy K, Takács M, Minarovits J (2016) Infectious Agents Associated with Head and Neck Carcinomas. Adv Exp Med Biol 897:63-80 
14. Kis A, Fehér E, Gáll T et al (2009) Epstein-Barr virus prevalence in oral squamous cell cancer and in potentially malignant oral disorders in an eastern Hungarian population. Eur $\mathbf{J}$ Oral Sci 117(5):536-540

15. Wu L, Feng J, Shi L et al (2013) Candidal infection in oral leukoplakia: a clinicopathologic study of 396 patients from eastern China. Ann Diagn Pathol 17(1):37-40

16. Hebbar PB, Pai A, D S (2013) Mycological and histological associations of Candida in oral mucosal lesions. J Oral Sci 55(2):157-160

17. Dilhari A, Weerasekera MM, Siriwardhana A et al (2016) Candida infection in oral leukoplakia: an unperceived public health problem. Acta Odontol Scand 74(7):565-569

18. Gainza-Cirauqui ML, Nieminen MT, Novak Frazer L et al (2013) Production of carcinogenic acetaldehyde by Candida albicans from patients with potentially malignant oral mucosal disorders. J Oral Pathol Med 42(3):243-249

19. Bakri MM, Cannon RD, Holmes AR et al (2014) Detection of Candida albicans ADH1 and ADH2 mRNAs in human archival oral biopsy samples. J Oral Pathol Med 43(9):704-710

20. Kazanowska-Dygdała M, Duś I, Radwan-Oczko M (2016) The presence of helicobacter pylori in oral cavities of patientsn with leukoplakia and oral lichenplanus. J Appl Oral Sci 24(1):18-23

21. Mizuki H, Kawamura T, Nagasawa D (2015) In situ immunohistochemical detection of intracellular mycoplasma salivarium in the epithelial cells of oral leukoplakia. J Oral PatholMed 44(2):134-144

22. Meisel P, Holtfreter B, Biffar R et al (2012) Association of periodontitis with the risk of oral leukoplakia. Oral Oncol 48(9):859-863

23. Hu X, Zhang Q, Hua H et al (2016) Changes in the salivary microbiota of oral leukoplakia and oral cancer. Oral Oncol 56:e6-e8

24. Hernandez BY, Zhu X, Goodman MT et al (2017) Betel nut chewing, oral premalignant lesions, and the oral microbiome. PLoS One 12(2):e0172196

25. Srinivas K, Aravinda K, Ratnakar P et al (2011) Oral lichen planus - Review on etiopathogenesis. Natl J Maxillofac Surg 2(1):15-16

26. Payeras MR, Cherubini K, Figueiredo MA et al (2013) Oral lichen planus: focus on etiopathogenesis. Arch Oral Biol 58(9):1057-1069

27. Georgakopoulou EA, Achtari MD, Achtaris M et al (2012) Oral lichen planus as a preneoplastic inflammatory model. J Biomed Biotechnol 2012:759626 
28. Roopashree MR, Gondhalekar RV, Shashikanth MC et al (2010) Pathogenesis of oral lichen planus-a review. J Oral Pathol Med 39(10):729-734

29. Gorouhi F, Davari P, Fazel N (2014) Cutaneous and mucosal lichen planus: a comprehensive review of clinical subtypes, risk factors, diagnosis, and prognosis. ScientificWorld Journal 2014:742826

30. Bäckman K, Jontell M (2007) Microbial-associated oral lichenoid reactions. Oral Dis 13(4):402-406

31. Taghavi Zenouz A, Mehdipour M, Jafari Heydarlou M et al (2010) Relationship between Lichen Planus and Helicobacter pylori Infection. J Dent Res Dent Clin Dent Prospects 4(1):17-20

32. Attia EA, Abdel Fattah NS, Abdella HM (2010) Upper gastrointestinal findings and detection of helicobacter pylori in patients with oral lichen planus. Clin Exp Dermatol 35(4):355-360

33. Pourshahidi S, Fakhri F, Ebrahimi H et al (2012) Lack of association between helicobacter pylori infection and oral lichen planus. Asian Pac J Cancer Prev 13(5):17451747

34. Izol B, Karabulut AA, Biyikoglu I et al (2010) Investigation of upper gastrointestinal tract involvement and $\mathrm{H}$. Pylori presence in lichen planus: a case-controlled study with endoscopic and histopathological findings. Int J Dermatol 49(10):1121-1126

35. Hulimavu SR, Mohanty L, Tondikulam NV et al (2014) No evidence for Helicobacter pylori in oral lichen planus. J Oral Pathol Med 43(8):576-578

36. Boorghani M, Gholizadeh N, Taghavi Zenouz A et al (2010) Oral lichen planus: clinical features, etiology, treatment and management; a review of literature. J Dent Res Dent Clin Dent Prospects 4(1):3-9

37. Ertugrul AS, Arslan U, Dursun R et al (2013) Periodontopathogen profile of healthy and oral lichen planus patients with gingivitis or periodontitis. Int J Oral Sci 5(2):92-97

38.Marchesi JR, Ravel J (2015) The vocabulary of microbiome research: a proposal. Microbiome 3:31

39. Papaioannou W, Gizani S, Haffajee AD, Quirynen M, Mamai-Homata E, Papagiannoulis L (2009) The microbiota on different oral surfaces in healthy children. Oral Microbiol Immunol 24(3):183-9

40. Zaura E, Keijser BJ, Huse SM, Crielaard W (2009) Defining the healthy "core microbiome" of oral microbial communities. BMC Microbiol 9:259 
41. Contreras M, Costello EK, Hidalgo G, Magris M, Knight R, Dominguez-Bello MG (2010) The bacterial microbiota in the oral mucosa of rural Amerindians. Microbiology 156:3282-7

42. Nagy K, Szőke I, Sonkodi I, Nagy E, Mari A, Szolnoky G, Newman HN (2000) Inhibition of microflora associated with oral malignancy. Oral Oncol 36(1):32-36

43. Hooper SJ, Crean SJ, Fardy MJ, Lewis MAO, Spratt DA, Wade WG, Wilson MJ (2007) A molecular analysis of the bacteria present within oral squamous cell carcinoma. J Med Microbiol 56(12):1651-1659

44. Lax AJ, Thomas W (2002) How bacteria could cause cancer: one step at a time. Trends Microbiol 10(6):294-299

45. Pushalkar S, Ji X, Li Y, Estilo C, Yegnanarayana R, Singh B, Li X, Saxena D (2012) Comparison of oral microbiota in tumor and non-tumor tissues of patients with squamous cell carcinoma. BMC Microbiol 12:144

46. Schmidt BL, Kuczynski J, Bhattacharya A, Huey B, Corby PM, Queiroz ELS, Nightingale K, Kerr AR, DeLacure MD, Veermachaneni R, Olshen A, Albertson DG (2014) Changes in abundance of oral microbiota associated with oral cancer. PLoS One 9 (6):e98741

47. Castellarin M, Warren RL, Freeman JD, Dreolini L, Krzywinski M, Strauss J, Barnes R, Watson P, Allen-Vercoe E, Moore RA, Holt RA (2012) Fusobacterium nucleatum infection is prevalent in human colorectal carcinoma. Genome Res 22(2):299-306

48. Kostic AD, Gevers D, Pedamallu CS, Michaud M, Duke F, Earl AM, Ojesina AI, Jung J, Bass AJ, Tabernero J, Baselga J, Liu C, Shivdasani RA, Ogino S, Birren BW, Huttenhower C, Garrett WS, Meyerson M (2012) Genomic analysis identifies association of Fusobacterium with colorectal carcinoma. Genome Res 22(2):292-298

49. Kostic AD, Chun E, Robertson L, Glickman JN, Gallini CA, Michaud M, Clancy TE, Chung DC, Lochhead P, Hold GL, El-Omar EM, Brenner D, Fuchs CS, Meyerson M, Garrett WS (2013) Fusobacterium nucleatum potentiates intestinal tumorigenesis and modulates the tumor-immune microenvironment. Cell Host Microbe 14(2):207-215

50. Bebek G, Bennett K, Funchain P, Campbell R, Seth R, Scharpf J, Burkey B, Eng C (2012) Microbiomic subprofiles and MDR1 promoter methylation in head and neck squamous cell carcinoma. Hum Mol Genet 21(7):1557-1565

51. Tahara T, Arisawa T, Shibata T, Yamashita H, Yoshioka D, Hirata I (2009) Effect of promoter methylation of multidrug resistance 1 (MDR1) gene on gastric carcinogenesis. Anticancer Res 29(1):337-341 
52. Nakashima K, Shigehara K, Kawaguchi S, Wakatsuki A, Kobori Y, Nakashima K, Ishii Y, Shimamura M, Sasagawa T, Kitagawa Y, Mizokami A, Namiki M (2014) Prevalence of human papillomavirus infection in the oropharynx and urine among sexually active men: a comparative study of infection by papillomavirus and other organisms, including Neisseria gonorrhoeae, Chlamydia trachomatis, Mycoplasma spp. and Ureaplasma spp. BMC Infect Dis $14: 43$

53. Henrich B, Rumming M, Sczyrba A, Velleuer E, Dietrich R, Gerlach W, Gombert M, Rahn S, Stove J Borkhardt A, Fischer U (2014) Mycoplasma salivarium as a dominant colonizer of Fanconi anemia associated oral carcinoma. PLoS One 9(3):e92297

54. Tsai S, Wear DJ, Shih JW, Lo SC (1995) Mycoplasmas and oncogenesis: persistent infection and multistage malignant transformation. Proc Natl Acad Sci U S A 92(22):1019710201

55. Feng SH, Tsai S, Rodriguez J, Lo SC (1999) Mycoplasma infections prevent apoptosis and induce malignant transformation of interleukin-3-dependent 32D hematopoietic cells. Mol Cell Biol 19(12):7995-8002

56. Zhang B, Shih JW, Wear DJ, Tsai S, Lo SC (1997) High level expression of H-ras and cmyc oncogenes in mycoplasma-mediated malignant cell transformation. Proc Soc Exp Biol Med 214(4):359-368

57. Mager DI, Haffajee AD, Devlin PM, Norris CM, Posner MR, Goodson JM (2005) The salivary microbiota as an indicator of oral cancer: a descriptive, non-randomized study of cancer free and oral squamous cell carcinoma subjects. J Transl Med 3:27

58. Pushalkar S, Mane SP, Ji X, Li Y, Evans C, Crasta OR, Morse D, Meagher RM, Singh A, Saxena D (2011) Microbial diversity in saliva of oral squamous cell carcinoma. FEMS Immunol Med Microbiol 61(3):269-277

59. Muto M, Hitomi Y, Ohtsu A, Shimada H, Kashiwase Y, Sasaki H, Yoshoda S, Esumi H (2000) Acetaldehyde production by non-pathogenic Neisseria in human oral microflora: implications for carcinogenesis in upper aerodigestive tract. Int J Cancer 88(3):342-350

60. Homann N, Tillonen J, Meurman JH, Rintama“ki H, Lindquist C, Rautio M, JousimiesSomer H, Salaspuro M (2000) Increased salivary acetaldehyde levels in heavy drinkers and smokers: a microbiological approach to oral cavity cancer. Carcinogenesis 21(4):663-668

61. Kurkivuori J, Salaspuro V, Kaihovaara P, Kari K, Rautemaa R, Gro“nroos L, Meurman J, Salaspuro M (2007) Acetaldehyde production from ethanol by oral streptococci. Oral Oncol 43(2):181-186

62. Homann N, Tillonen J, Rintama"ki H, Salaspuro M, Lindquist C, Meurman JH (2001) Poor dental status increases acetaldehyde production from ethanol in saliva: a possible link to increased oral cancer risk among heavy drinkers. Oral Oncol 37(2):153-158 
63. Hsiao JR, Chang CC, Lee WT, Huang CC, Ou CY, Tsai ST, Chen KC, Huang JS, Wong TY, Lai YH, Wu YH, Hsueh WT, Wu SY, Yen CJ, Chang JY, Lin CL, Weng YL, Yang HC, Chen YS, Chang JS (2018) The interplay between oral microbiome, lifestyle factors and genetic polymorphisms in the risk of oral squamous cell carcinoma. Carcinogenesis 39(6):778-787

64 Lee J, Roberts JS, Atanasova KR, Chowdhury N, Han K, Yilmaz Ö (2017) Human Primary Epithelial Cells Acquire an Epithelial-Mesenchymal-Transition Phenotype during Long-Term Infection by the Oral Opportunistic Pathogen, Porphyromonas gingivalis. Front Cell Infect Microbiol 7:493

65. Al-Hebshi NN, Nasher AT, Maryoud MY, Homeida HE, Chen T, Idris AM, Johnson NW (2017) Inflammatory bacteriome featuring Fusobacterium nucleatum and Pseudomonas aeruginosa identified in association with oral squamous cell carcinoma. Sci Rep 7(1):1834

66. Perera M, Al-Hebshi NN, Speicher DJ, Perera I, Johnson NW (2016) Emerging role of bacteria in oral carcinogenesis: a review with special reference to perio-pathogenic bacteria. $\mathrm{J}$ Oral Microbiol 8:32762

67. Rubinstein MR, Wang X, Liu W et al (2013) Fusobacterium nucleatum promotes colorectal carcinogenesis by modulating Ecadherin/ $\beta$-catenin signaling via its FadA adhesion. Cell Host Microbe 14(2):195-206

68. Schmidt BL, Kuczynski J, Bhattacharya A et al (2014) Changes in abundance of oral microbiota associated with oral cancer. PLoS One 9(6):e98741

69. Watts GS, Youens-Clark K, Slepian MJ, Wolk DM, Oshiro MM, Metzger GS, Dhingra D, Cranmer LD, Hurwitz BL (2017) 16S rRNA gene sequencing on a benchtop sequencer: accuracy for identification of clinically important bacteria. J Appl Microbiol 123(6):15841596

70. Chakravorty S, Helb D, Burday M, Connell N, Alland D (2007) A detailed analysis of $16 \mathrm{~S}$ ribosomal RNA gene segments for the diagnosis of pathogenic bacteria. J Microbiol Methods 69(2):330-9

71. Whiteley AS, Jenkins S, Waite I, Kresoje N, Payne H, Mullan B, Allcock R, O'Donnell A (2012) Microbial 16S rRNA Ion Tag and community metagenome sequencing using the Ion Torrent (PGM) Platform. J Microbiol Methods 91(1):80-8

72. Clark AE, Kaleta EJ, Arora A, Wolk DM (2013) Matrix-assisted laser desorption ionization-time of flight mass spectrometry: a fundamental shift in the routine practice of clinical microbiology. Clin Microbiol Rev 26(3):547-603

73. Sharma PK, Capalash N, Kaur J (2007) An improved method for single step purification of metagenomic DNA. Mol Biotechnol 36(1):61-63 
74. Nagy E. et al. (2009) Species identification of clinical isolates of Bacteroides by matrixassisted laser-desorption-ionization time-of-flight mass spectrometry. Clinical Microbiology and Infection 15: 796-802.

75. Periasamy S, Chalmers NI, Du-Thumm L et al (2009) Fusobacterium nucleatum ATCC 10953 requires Actinomyces naeslundii ATCC 43146 for growth on saliva in a three-species community that includes Streptococcus oralis 34. Appl Environ Microbiol 75(10):3250-3257

76. El-Naggar AK, Chan JKC, Takata T, Grandis JR, Slootweg PJ (2017) Tumours of the oral cavity and mobile tongue In: The fourth edition of the head and neckWorld Health Organization blue book: editors' perspectives. Hum Pathol 66:10-12

77. Harmati M, Gyukity-Sebestyen E, Dobra G, Terhes G, Urban E, Decsi G, Mimica-Dukić N, Lesjak M, Simin N, Pap B, Nemeth IB, Buzas K (2017) Binary mixture of Satureja hortensis and Origanum vulgare subsp. hirtum essential oils: in vivo therapeutic efficiency against Helicobacter pylori infection. Helicobacter 22(2)

78. Müller S (2018) Oral epithelial dysplasia, atypical verrucous lesions and oral potentially malignant disorders: focus on histopathology. Oral Surg Oral Med Oral Pathol Oral Radiol 125(6):591-602

79. Arduino PG, Romano F, Sasia D et al (2017) Subgingival microbiota in white patients with Desquamative gingivitis: a cross-sectional study. J Periodontol 88(7):643-650

80. Kreth J, Merritt J, Qi F (2009) Bacterial and host interactions of oral streptococci. DNA Cell Biol 28(8):397-403

81. Van Hoogmoed CG, Geertsema-Doornbusch GI, Teughels Wet al (2008) Reduction of periodontal pathogens adhesion by antagonistic strains. Oral Microbial Immunol 23(1):43-48

82. Standar K, Kreikemeyer B, Redanz S et al (2010) Setup of an in vitro test system for basic studies on biofilm behavior of mixedspecies cultures with dental and periodontal pathogens. PLoS One 5(10):e13135

83. Otsuki K, Imai N (2017) Effects of lactoferrin in 6 patients with refractory bacterial vaginosis. Biochem Cell Biol 95(1):31-33

84. Klarić M, Mandić V, Lovrić S, e al (2017) Antimicrobial efficacy of probiotic-containing toothpastes: an in vitro evaluation. Med Glas (Zenica) 14(1):16-24

85. Wu J, Li Q, Fu X (2019) Fusobacterium nucleatum Contributes to the Carcinogenesis of Colorectal Cancer by Inducing Inflammation and Suppressing Host Immunity. Transl Oncol 12(6):846-851

86. Brennan CA, Garrett WS (2019) Fusobacterium nucleatum - symbiont, opportunist and oncobacterium. Nat Rev Microbiol 17(3):156-166 
87. Mizuki H, Abe R, Mikami T (2017) Ultrastructural Changes during the Life Cycle of Mycoplasma salivarium in Oral Biopsies from Patients with Oral Leukoplakia. Front Cell Infect Microbiol 21(7):403

88. Singh N, Baby D, Rajguru JP, Patil PB, Thakkannavar SS, Pujari VB (2019) Inflammation and cancer. Ann Afr Med 18(3):121-126

89. Lehours P, Ferrero RL (2019) Review: Helicobacter: Inflammation, immunology, and vaccines. Helicobacter 24:e12644

90. Peng Q, Zhang J, Ye X, Zhou G (2017) Tumor-like microenvironment in oral lichen planus: evidence of malignant transformation? Expert Rev Clin Immunol 13(6):635-643

91. Otero-Rey EM, Suarez-Alen F, Peñamaria-Mallon M, Lopez-Lopez J, Blanco-Carrion A (2014) Malignant transformation of oral lichen planus by a chronic inflammatory process. Use of topical corticosteroids to prevent this progression? Acta Odontol Scand 72(8):570-7

92. Liu Y, Messadi DV, Wu H, Hu S (2010) Oral lichen planus is a unique disease model for studying chronic inflammation and oral cancer. Med Hypotheses 75(6):492-4

93. Castillo GDV, Blanc SL, Sotomayor CE, Azcurra AI (2018) Study of virulence factor of Candida species in oral lesions and its association with potentially malignant and malignant lesions. Arch Oral Biol 91:35-41

94. Baek K, Choi Y (2018) The microbiology of oral lichen planus: Is microbial infection the cause of oral lichen planus? Mol Oral Microbiol 33(1):22-28

95. Yu S, Yang M, Nam KT (2014) Mouse models of gastric carcinogenesis. J Gastric Cancer. 14(2):67-86

96. Irani S, Monsef Esfahani A, Bidari Zerehpoush F (2013) Detection of Helicobacter pylori in Oral Lesions. J Dent Res Dent Clin Dent Prospects 7(4):230-7

97. Murata M (2018) Inflammation and cancer. Environ Health Prev Med 23(1):50

98. Syrjänen S, Lodi G, von Bültzingslöwen I, Aliko A, Arduino P, Campisi G, et al. (2011) Human papillomaviruses in oral carcinoma and oral potentially malignant disorders: a systematic review. Oral Dis 17:58-72.

99. Szarka K, Tar I, Fehér E, Gáll T, Kis A, Tóth ED, Boda, et al. (2009) Progressive increase of human papillomavirus carriage rates in potentially malignant and malignant oral disorders with increasing malignant potential. Oral Microbiol Immunol 24:314-8.

100. Syrjanen S, Syrjanen K. (2000) HPV infections of the oral mucosa, Chapter 17. In: Syrjanen K, Syrjanen S, Eds. Papillomavirus infections in human pathology. New York: J Wiley \& Sons 379-412. 
101. Nielsen H, Norrild B, Vedtofte P, Praetorius F, Reibel J, Holmstrup P. (1996) Human papillomavirus in oral premalignant lesions. Eur J Cancer B Oral Oncol 32B:264-70

102. Kumaraswamy KL, Vidhya M. (2011) Human papilloma virus and oral infections: an update. J Cancer Res Ther 7:120-7 


\section{Appendix}

Copies of the publications providing the basis of the thesis

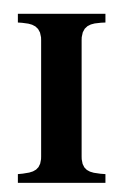




\title{
Chicken or the Egg: Microbial Alterations in Biopsy Samples of Patients with Oral Potentially Malignant Disorders
}

\author{
Gabor Decsi ${ }^{1}$ - Jozsef Soki ${ }^{2}$ - Bernadett Pap ${ }^{3}$. Gabriella Dobra ${ }^{3}$ - Maria Harmati ${ }^{3}$ - Sandor Kormondi ${ }^{4}$. \\ Tibor Pankotai ${ }^{5}$. Gabor Braunitzer ${ }^{6}$. Janos Minarovits ${ }^{7}$. Istvan Sonkodi ${ }^{1}$. Edit Urban ${ }^{2}$ • Istvan Balazs Nemeth ${ }^{8}$. \\ Katalin Nagy ${ }^{1} \cdot$ Krisztina Buzas $^{3,7}$
}

Received: 8 June 2018 / Accepted: 19 July 2018

(C) Arányi Lajos Foundation 2018

\begin{abstract}
Oral carcinogenesis often leads to the alteration of the microbiota at the site of the tumor, but data are scarce regarding the microbial communities of oral potentially malignant disorders (OPMDs). Punch biopsies were taken from healthy and nonhealthy mucosa of OPMD patients to analyze the microbiome using metagenome sequencing. In healthy oral mucosa biopsies the bacterial phyla Firmicutes, Fusobacteria, Proteobacteria, Actinobacteria and Bacteroidetes were detected by Ion Torrent sequencing. The same phyla as well as the phyla Fibrobacteres and Spirochaetes were present in the OPMD biopsies. On the species level, there were 10 bacterial species unique to the healthy tissue and 35 species unique to the OPMD lesions whereas eight species were detected in both samples. We observed that the relative abundance of Streptococcus mitis decreased in the OPMD lesions compared to the uninvolved tissue. In contrast, the relative abundance of Fusobacterium nucleatum, implicated in carcinogenesis, was elevated in OPMD. We detected markedly increased bacterial diversity in the OPMD lesions compared to the healthy oral mucosa. The ratio of $S$. mitis and $F$. nucleatum are characteristically altered in the OPMD lesions compared to the healthy mucosa.
\end{abstract}

Keywords OPMD $\cdot$ Oral microbiome $\cdot$ Metagenome sequencing $\cdot$ Lichen $\cdot$ Leukoplakia

Krisztina Buzas

kr.buzas@gmail.com; buzas.krisztina@brc.mta.hu

1 Faculty of Dentistry, Department of Oral Surgery, University of Szeged, Tisza Lajos krt. 64, Szeged H-6720, Hungary

2 Albert Szent-Gyorgyi Clinical Centre, Institute of Clinical Microbiology, University of Szeged, Semmelweis u. 6, Szeged H-6725, Hungary

3 Biological Research Centre, Hungarian Academy of Sciences, Temesvari krt. 62, Szeged H-6726, Hungary

4 Albert Szent-Gyorgyi Clinical Centre, Department of Traumatology, University of Szeged, Semmelweis u. 6, Szeged H-6725, Hungary

5 Department of Biochemistry and Molecular Biology, University of Szeged, Kozep fasor 52, Szeged H-6726, Hungary

6 dicomLAB Ltd., Pulz u. 46/b, Szeged H-6724, Hungary

7 Faculty of Dentistry, Department of Oral Biology and Experimental Dental Research, University of Szeged, Tisza Lajos krt. 64, Szeged H-6720, Hungary

8 Department of Dermatology and Allergology, University of Szeged, Szeged H-6720, Hungary

\section{Introduction}

Numerous scientific data demonstrate the altered bacterial colonization of cancerous tissue, but the causality of microbial alteration has not clarified yet.

The role of oral microbes in the development of oral potentially malignant disorder (OPMD) and oral squamous cell carcinoma (OSCC) is periodically reevaluated, since OPMD or OSCC may develop in the absence of the traditional risk factors like smoking, alcohol consumption and betel nut use [1-3]. While the microbiological background of oral squamous cell carcinoma was intensively studied in the last two decades, less attention has been paid to OPMD in this respect [4].

OPMD is a group of disorders of diverse etiologies, frequently associated with tobacco consumption and mutations in the DNA of oral epithelial cells. A fraction of OPMD undergoes clinical and histomorphological alterations that lead to the development of OSCC. These disorders include leukoplakia, erythroplakia, oral lichen planus, submucous fibrosis, and actinic cheilitis [5, 6]. In addition, inherited cancer syndromes such as xeroderma pigmentosum and Fanconi's 
anemia are also associated with an increased incidence of malignant tumors, among them oral carcinoma [6].

Leukoplakia is defined as "A white plaque of questionable risk having excluded (other) known diseases or disorders that carry no increased risk for cancer". Leukoplakia is six times more common among smokers than among non-smokers [6]. These lesions are divided into homogenous (simplex) and non-homogenous types. The non-homogenous types based on the cellular variability are verrucous leukoplakia, nodular leukoplakia and erythroleukoplakia [7]. Proliferative verrucous leukoplakia (PVL) is a subtype of verrucous leukoplakia that shows resistance to treatment and a high rate of malignant transformation. It is more frequent among elderly women, in many cases without smoking in the anamnesis. Distinct histopathological changes, like hyperkeratosis with or without dysplasia, may accompany the transition of PVL to verrucous hyperplasia and verrucous carcinoma [6].

There are conflicting results regarding the association of leukoplakia and human papillomavirus (HPV) infection $[6,8]$. The role of torque teno virus (TTV) [9, 10], Epstein-Barr virus (EBV) [11] and Candida albicans $[8,12-16]$ in leukoplakia development and carcinogenesis remains to be clarified, too. Additionally, it has been also demonstrated that specific bacterial infections like Helicobacter pylori [17] or the intracellular Mycoplasma salivarium [18] could also be involved in this process.

A disbalance in the oral microbial flora can be also accompanied with leukoplakia and carcinogenesis, as suggested by the association of periodontal inflammation with leukoplakia [19] and a changing bacterial flora in the saliva and on the oral mucosal surfaces of patients with OPMD [20,21].

Lichen planus (LP) is a chronic, idiopathic, inflammatory disease of the oral mucosa or the skin, presenting as a white lesion when it affects the oral cavity (oral lichen planus, OLP). A crucial aspect of the pathomechanism of OLP is the accumulation of CD8+ T lymphocytes under the basal cell layer of the oral mucosa, which causes DNA damage and the apoptosis of the keratinocytes by antigen-specific cell-mediated immune response, and also basement membrane degradation [22-25]. According to the most accepted hypothesis, chronic stimulation from the inflammatory and stromal cells provides the initial signal which lead to the abolished growth control of epithelial cells. Additionally, oxidative stress induced DNA damage could also lead to neoplastic changes, but the initial event leading to this signal cascade activation has not been characterized yet. Based on the increasing evidence viral, fungal, and bacterial antigens have all been suggested [26-34] as a potential initiating factor in LP. If there is a relationship between the bacterial flora and OLP, the question is whether the trigger area is in the oral cavity, or at another area of the body, such as the skin, the gastrointestinal tract, the larynx or the eyes. If oral bacterial infection could initiate OLP development, it is not clear whether a single bacterial species could initiate the OLP transformations, or is it the interaction of several species during the process? Additionally, the disturbed balance of the normal bacterial flora could also be involved in the initial steps of OLP activation.

In our experimental setup, we examined the oral microbiome of patients diagnosed with OPMD. We compared the microbiome of healthy and non-healthy mucosal surfaces. Using metagenome sequencing, we detected markedly increased bacterial diversity in the OPMD lesions compared to the healthy oral mucosa. In parallel, in the OPMD lesions there was a reduced representation of distinct Streptococcus species that dominate the bacterial community of healthy oral mucosa.

\section{Materials and Methods}

\section{Patient Selection}

Potential participants were interviewed by the clinical coordinators at the University of Szeged, Faculty of Dentistry. Every potential participant was informed about the ethical permission of the study and received both written and oral information on the goals and procedures of this study. The initial participants were selected by volunteering activity and the detailed questionnaire on family anamnesis, chronic diseases, medication-, alcohol-, tobacco- and drug consumption, oral hygiene, and sexual habits was filled out by the volunteers. Eligibility was determined based on the results of this questionnaire. Female and male subjects over 18 years of age were eligible for the study, provided that they were able to provide signed and dated informed consent and if they did not meet any of the exclusion criteria. As for the patient group, an established diagnosis of OPMD was also a requirement. The exclusion criteria included vital signs outside the acceptable range at the screening visit (i.e., blood pressure $>140$ / $90 \mathrm{mmHg}$, oral temperature $>37{ }^{\circ} \mathrm{C}$, heart rate $>100 /$ min), pregnancy, the potential subject being a sex worker, topical antibiotic treatment up to 7 days before the screening visit, and the use of the following drugs within 2 months before the screening visit: systemic antibiotics, corticosteroids, cytokines, methotrexate or immunosuppressive cytotoxic agents, or large doses of com-

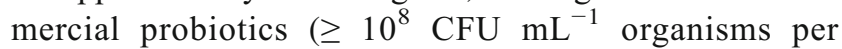
day). No patients were on specific diet, nor on antibiotic therapy in the previous 6 months. The clinical characteristics and brief medical history of the patient group is given in Table 1.

The study protocol conformed to the Declaration of Helsinki in all respects and was approved by the Institutional Ethics Committee of the University of Szeged (No. 3161). 


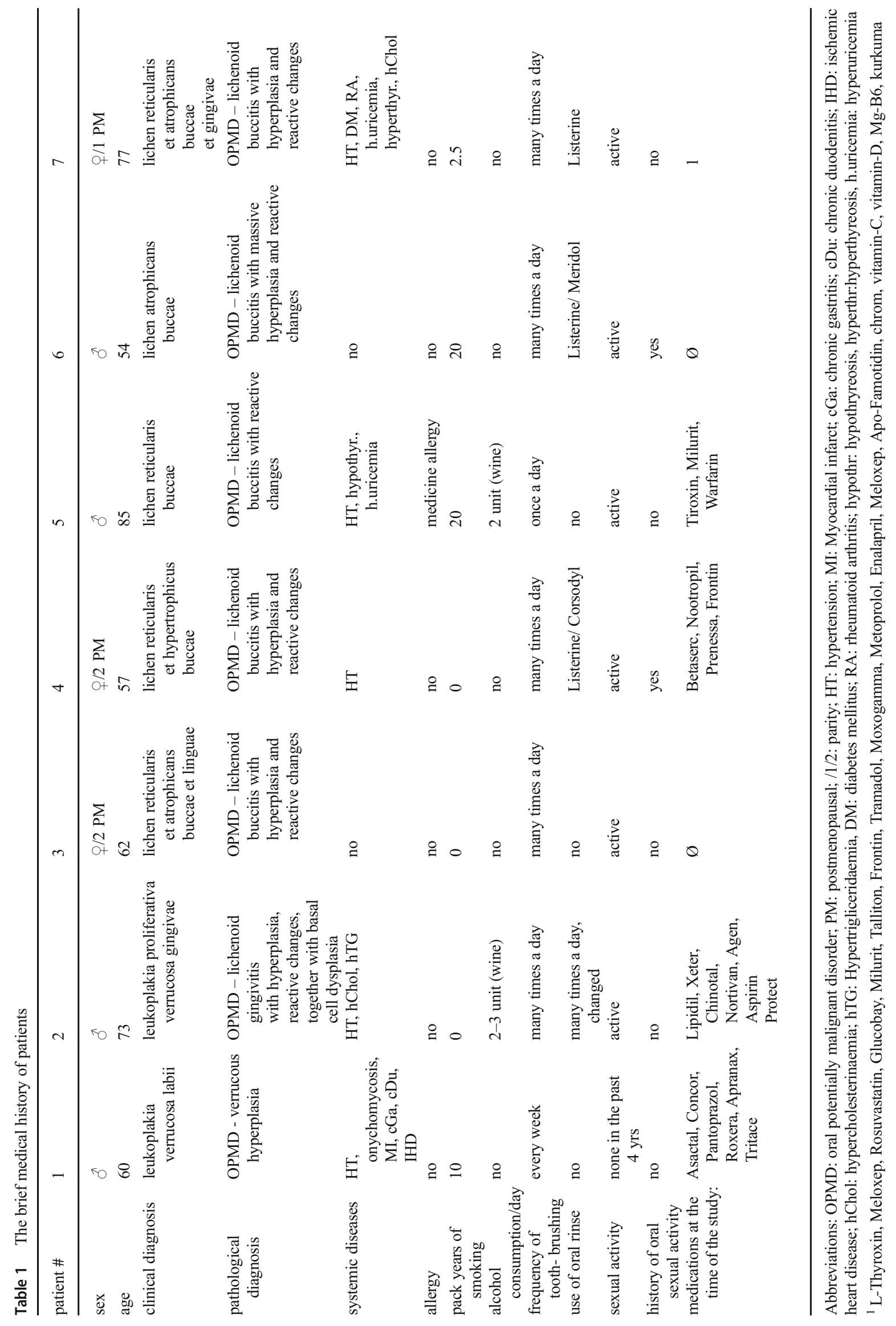


Table 2 The histopathological characteristics of the biopsies

\begin{tabular}{llllllll}
\hline patient \# & 1 & 2 & 3 & 4 & 5 & 6 & 7 \\
\hline OPMD & + & + & + & + & + & + & + \\
hyperkeratosis & + & ++ & +++ & +++ & + & +++ & +++ \\
hyperplasia & +++ & ++ & + & +++ & - & +++ & +++ \\
reactive atypia & - & + & - & + & - & + & + \\
dysplasia & - & + & - & - & - & - & - \\
lichenoid infiltrate & + & +++ & ++ & +++ & +++ & +++ & ++ \\
MT & - & - & - & - & - & - & - \\
\hline
\end{tabular}

Abbreviations: $(-$ no, + mild, ++ moderate, +++ severe; OPMD: oral potentially malignant disorder; MT: malignant transformation)

\section{Tissue Sample Collection for Metagenome Sequencing, Histopathological Assessment}

As a classical method for tissue sample collection from mucosal lesions in the oral cavity, we used punch biopsy. The biopsy was made with punches $4 \mathrm{~mm}$ in diameter. We collected 2 identical samples from the intralesional area: one sample was taken for hematoxylin-eosin (HE) staining, and an additional sample for metagenome sequencing. Additionally, we also collected a third, control specimen from the ipsilateral healthy mucosa for metagenome sequencing. The specimens contained the mucosa, and submucosal connective tissue. Samples for metagenome sequencing were collected in Eppendorf tubes filled with $20 \mathrm{mM}$ potassium phosphate buffer ( $\mathrm{pH} 7.0$ ), and stored at $-20^{\circ} \mathrm{C}$. Tissue samples for HE staining were fixed in

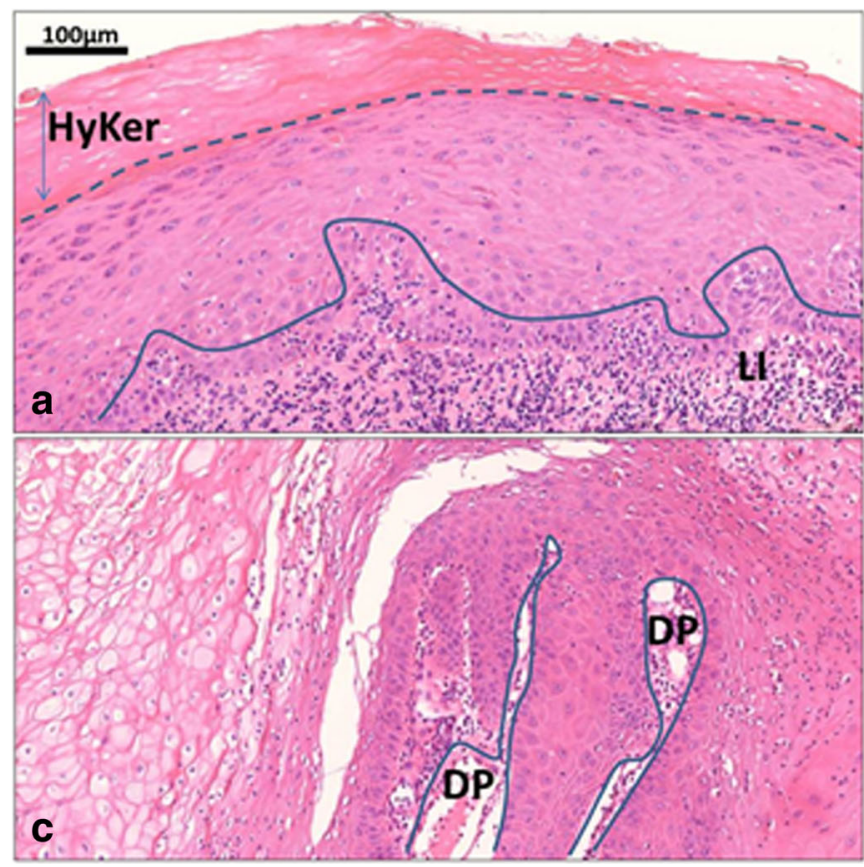

Fig. 1 Representative pictures from OPMD tissue samples OPMD tissue samples with marked hyperkeratosis (a, b - HyKer), acanthosis and lichenoid infiltrate (a, d - LI). Occasionally there was a marked verrucous hyperplasia with elongated dermal papillae $(\mathbf{c}-\mathrm{DP})$. Note formaldehyde solution, and sent for histopathological assessment. Pathological dataset (Table 2) included the absence or presence, together with severity of hyperkeratosis, hyperplasia, reactive atypia, dysplasia, and lichenoid infiltrate [35].

\section{Metagenomic DNA Isolation}

Total DNA was isolated from patient samples as described previously, with minor modifications [36]. Briefly, samples $(600 \mu \mathrm{L})$ were suspended in $650 \mu \mathrm{L}$ of extraction buffer (100 mM TrisCl, $\mathrm{pH} 8.0,100 \mathrm{mM}$ EDTA, $\mathrm{pH} 8.0,1.5 \mathrm{M} \mathrm{NaCl}, 100 \mathrm{mM}$ sodium phosphate, $\mathrm{pH} 8.0,1 \%$ CTAB) $3.5 \mu \mathrm{L}$ proteinase $\mathrm{K}$ (20.2 $\mathrm{mg} \mathrm{mL}^{-1}$ ) and incubated horizontally at $37{ }^{\circ} \mathrm{C}$ for $45 \mathrm{~min}$, next $80 \mu \mathrm{L}$ of $20 \%$ SDS was added and mixed by inversion for several times with further incubation at $60{ }^{\circ} \mathrm{C}$ for $1 \mathrm{~h}$. The sample in each tube was mixed thoroughly every $15 \mathrm{~min}$. The particles were collected by centrifugation $(17,000 \mathrm{~g})$ for $5 \mathrm{~min}$. The supernatant was transferred into clean tubes and was mixed with equal quantity of phenol chloroform and isoamyl alcohol $(25: 24: 1)$ and extracted three times. DNA was precipitated with 0.7 volume isopropanol, the pellet was washed with $70 \%$ ethanol. Crude DNA pellets were dried and dissolved in $50 \mu \mathrm{L}$ of TE buffer (10 mM Tris-HCl, $1 \mathrm{mM}$ sodium EDTA, $\mathrm{pH}$ 8.0). Metagenomic DNA was quantified using Qubit ${ }^{\circledR} 2.0$ Fluorometer. Half of total metagenomic DNA from the

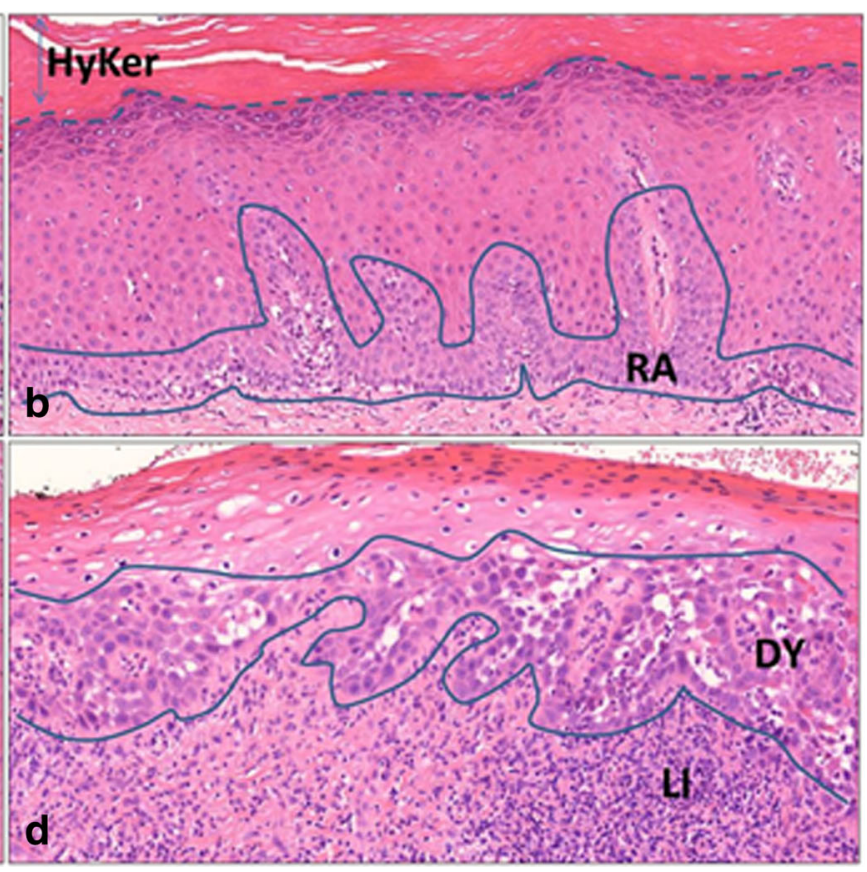

the regenerative basal cell changes (b-RA/regenerative atypia/), in contrast to mild dysplasia (d - DY) with focal acantholysis /HE; OM $112 \times 1$ 
Fig. 2 Distribution of taxonomic domains Significant differences were not found in the distributions of taxonomic domains between the healthy tissue and the OPMD lesion. $6.78 \%$ of bacterial domains were detected in healthy area compared $6.87 \%$ of bacterial domains in white lesions of oral mucosa

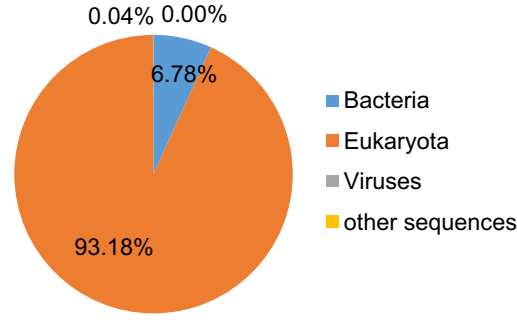

Healthy

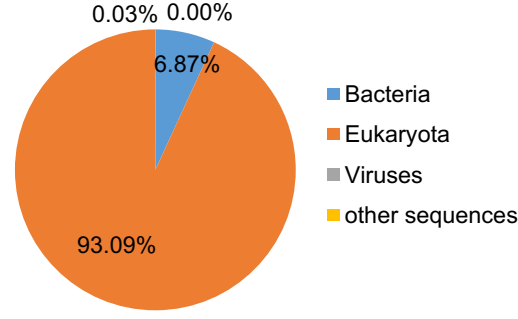

OPMD healthy and lesion samples were pooled and stored at $-20{ }^{\circ} \mathrm{C}$ for sequencing.

\section{Library Preparation and Sequencing}

Ion Torrent PGM Fragment libraries of $200 \mathrm{nt}$ were generated according to the appropriate protocols (Ion Torrent PGM, Life Tech, USA). $1 \mu \mathrm{g}$ pooled metagenomic DNA from each sample was used for library preparation for each sample. DNA shearing and end-repair was achieved by Ion Xpress ${ }^{\mathrm{TM}}$ Plus Fragment Library Kit, and DNA Purification was carried out by PureLink PCR Purification Kit (Thermo Fisher Scientific, MA). Adapter ligation and nick translation were performed by Ion Shear Plus Reagents Kit (Thermo Fisher Scientific, MA). Size selection was performed in $2 \%$ agarose gel to enrich the 300-350 nt fragments then library amplification was achieved by using Platinum ${ }^{\circledR}$ PCR SuperMix (Thermo Fisher Scientific, MA). ION Library TaqMan qPCR was used for quantitation and Ion Xpress Template 200 ePCR kit was used for the emulsion PCR. Sequencing was performed on Ion Torrent Personal Genome Machine ${ }^{\mathrm{TM}}$ using Ion 318 chip. Ion Torrent Personal Genome Machine ${ }^{\mathrm{TM}}$ sequencing resulted 872,798 sequence reads for sample 1 (control) with an average read length of $219 \pm 71$ bases and 644,200 sequence reads for sample 2 (diseased) with an average read length of $220 \pm 72$ bases.

\section{Quality Assurance}

The MG-RAST software performs a QC (quality control) and an automatic normalization of the FASTQ sequence. For the taxonomical analyses, maximum e-value cut-off of $10^{-15}$, minimum percent identity cut-off $90 \%$ and minimum alignment length cut-off $40 \mathrm{nt}$ settings were applied. The overall community composition was determined using the M5nr database. Hits for the eukaryotic data were removed and relative abundance of the bacterial data was calculated.

\section{Swab Sample Collection}

We used cotton swabs for collecting bacterial samples. Two individual samples were taken from each patient, one from the surface of the lesion, and another one from the ipsilateral healthy (non-involved) mucosa. The swabs were rolled 4 times over the chosen areas. Then the swabs were placed in anaerobic
Fig. 3 Bacterial diversity a Metagenome sequencing revealed that the bacterial diversity in the OPMD biopsies was higher compared to the healthy oral mucosa. Within the Bacteria domain Firmicutes,

Fusobacteria, Proteobacteria, Actinobacteria, Bacteroidetes phyla were present in the healthy oral mucosa. In the OPMD biopsies the same phyla were identified in descending order of relative abundance; however, two additional phyla, Fibrobacteres and Spirochaetes were observed, too. b Metagenome sequencing detected 18 different bacterial species in healthy tissue and 43 species in the OPMD lesion. Eight bacterial species were detected in both samples
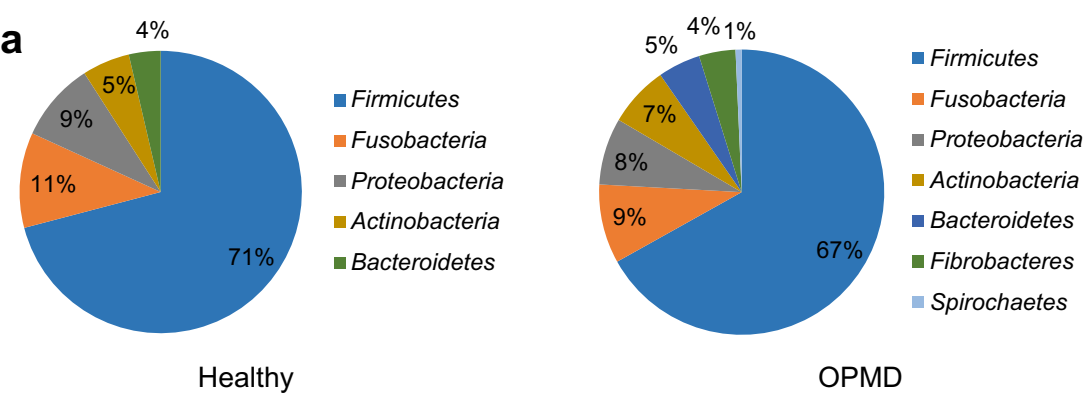

b

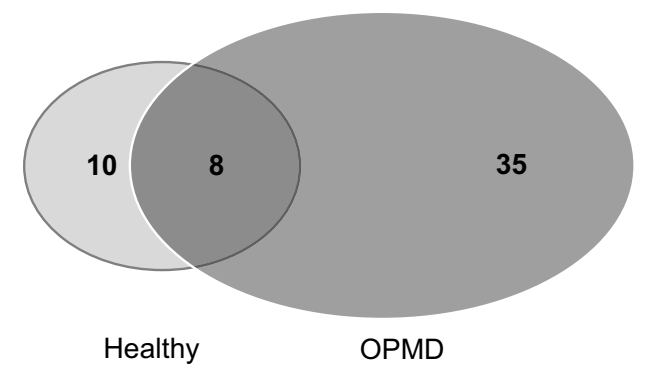


transport medium (AnaerobeSystems, CA) and sent immediately to the Institute of Clinical Microbiology, University of Szeged, for Fusobacterium nucleatum cultivation.

\section{Fusobacterium nucleatum qPCR}

DNA templates for qPCR were prepared from the undiluted BHI suspensions of the oral swabs by the QiAmp Stool Mini DNA Kit (Qiagen, Germany) as recommended by the supplier. Subsequent quantitative RT-PCRs for Fusobacterium nucleatum were done in StepOne RT-PCR instrument (Invitrogen, CA) using $5 \mu \mathrm{L}$ Brilliant II master mix (Agilent), primers FnucF CTTAGGAATGAGACAGAGATG and FnucR TGATGGTAACATACGAAAGG $0.2 \mu \mathrm{L}$ (35 pmole $/ \mu \mathrm{L}$ ) each, $1 \mu \mathrm{L}$ of template sample and the following cycling conditions: starting denaturation and hot start activation $95{ }^{\circ} \mathrm{C} 10 \mathrm{~min}$, $95^{\circ} \mathrm{C} 15 \mathrm{~s}, 56^{\circ} \mathrm{C} 15 \mathrm{~s}$ and $72^{\circ} \mathrm{C} 30 \mathrm{~s}, 40 \times$; and a melting curve from $72{ }^{\circ} \mathrm{C}$ to $95^{\circ} \mathrm{C}$ [37] $\mathrm{CFUs}$ were calculated comparing the means of threshold cycles to ones of a F. nucleatum 10-fold serial dilution samples prepared with the same kit.

\section{Data Analysis}

For the comparison of the numerical data, the Mann-Whitney U test was used in SPSS 21.0 (IBM, NY).

\section{Results}

Sample selection was based on the histological analysis of oral potentially malignant disorder. Figure 1 shows representative pictures of the OPMD tissue samples with marked hyperkeratosis, acanthosis and lichenoid infiltration. Table 1. describes the brief medical history of selected patients (Selection criteria has been found in Materials and methods). Detailed histological classification summarized in Table 2.

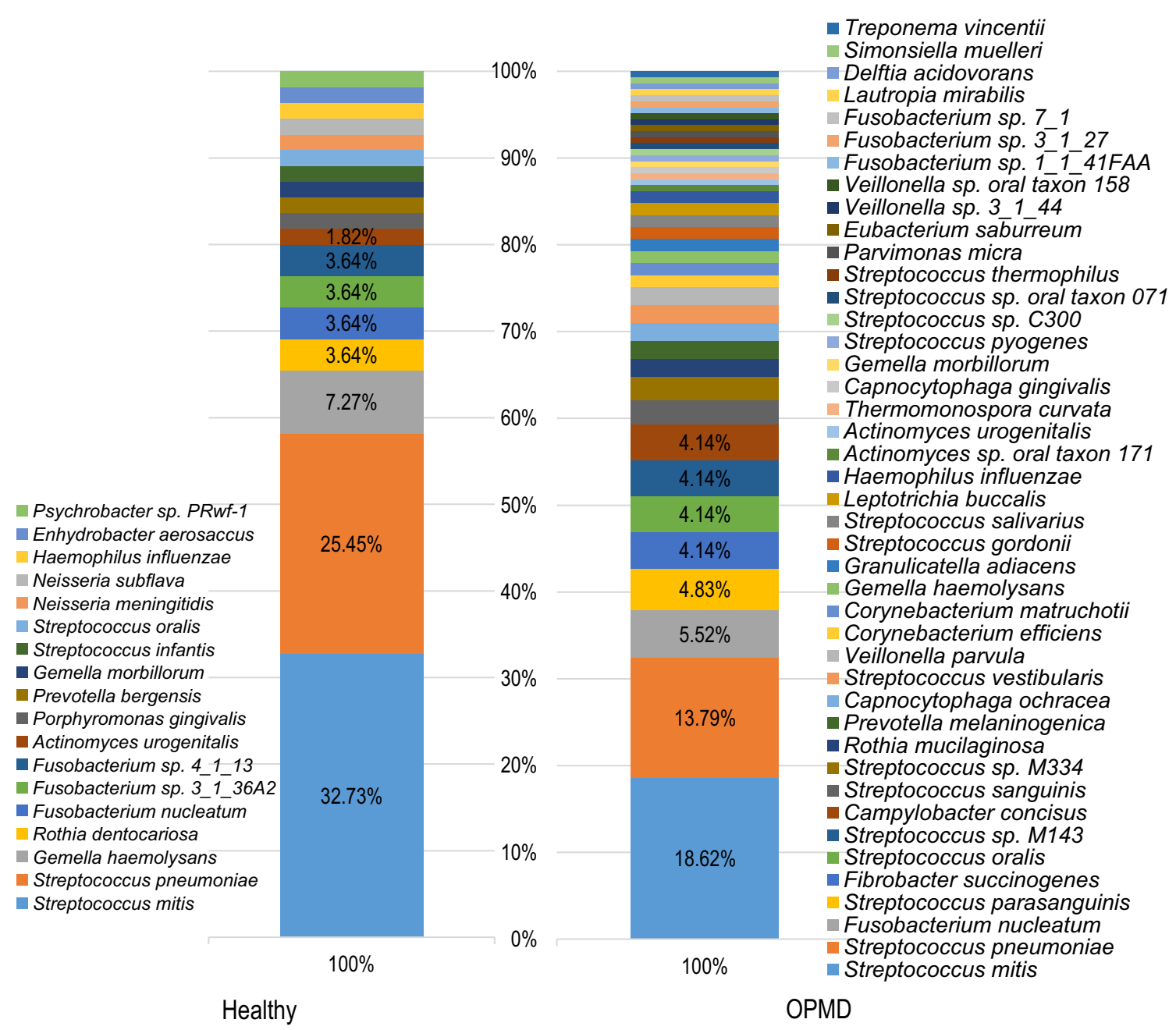

Fig. 4 Comparison of the detected bacterial species Metagenome sequencing detected 18 different bacterial species in healthy tissue and 43 species in the OPMD lesion 
First, we determined the ratio of bacterial DNA in our sample. As punch biopsy samples contain a high amount of human tissue, this step helped us to define the limitation of the study. In healthy oral mucosa samples, $6.78 \%$ of the sequence reads were annotated to the domain Bacteria. The ratio of bacterial sequences was similar $(6.87 \%)$ in the pooled OPMD DNA samples (Fig. 2).

Metagenome sequencing revealed that the bacterial diversity in the OPMD biopsies was higher compared to the healthy oral mucosa. Within the Bacteria domain Firmicutes, Fusobacteria, Proteobacteria, Actinobacteria, Bacteroidetes phyla were present in the healthy oral mucosa. In the OPMD biopsies the same phyla, i.e. Firmicutes, Fusobacteria, Proteobacteria, Actinobacteria and Bacteroidetes were identified in descending order of relative abundance; however, two additional phyla, Fibrobacteres and Spirochaetes were observed, too (Fig. 3a).

Table 3 Comparison of bacterial diversity in the healthy mucosa and the OPMD lesion

\begin{tabular}{|c|c|c|c|}
\hline Species & Phylum & Healthy & OPMD \\
\hline Streptococcus mitis & Firmicutes & $32,73 \%$ & $18,62 \%$ \\
\hline Streptococcus pneumoniae & Firmicutes & $25,45 \%$ & $13,79 \%$ \\
\hline Gemella haemolysans & Firmicutes & $7,27 \%$ & $1,38 \%$ \\
\hline Rothia dentocariosa & Actinobacteria & $3,64 \%$ & 0,021 \\
\hline Fusobacterium nucleatum & Fusobacteria & $3,64 \%$ & $5,52 \%$ \\
\hline Actinomyces urogenitalis & Actinobacteria & $1,82 \%$ & $0,69 \%$ \\
\hline Streptococcus oralis & Firmicutes & $1,82 \%$ & $4,14 \%$ \\
\hline Haemophilus influenzae & Proteobacteria & $1,82 \%$ & $1,38 \%$ \\
\hline Fusobacterium sp. 3_1_36A2 & Fusobacteria & $3,64 \%$ & 0 \\
\hline Fusobacterium sp. 4_1_13 & Fusobacteria & $3,64 \%$ & 0 \\
\hline Porphyromonas gingivalis & Bacteroidetes & $1,82 \%$ & 0 \\
\hline Prevotella bergensis & Bacteroidetes & $1,82 \%$ & 0 \\
\hline Gemella morbillorum & Firmicutes & $1,82 \%$ & 0 \\
\hline Streptococcus infantis & Firmicutes & $1,82 \%$ & 0 \\
\hline Neisseria meningitidis & Proteobacteria & $1,82 \%$ & 0 \\
\hline Neisseria subflava & Proteobacteria & $1,82 \%$ & 0 \\
\hline Enhydrobacter aerosaccus & Proteobacteria & $1,82 \%$ & 0 \\
\hline Psychrobacter sp. PRwf-1 & Proteobacteria & $1,82 \%$ & 0 \\
\hline Streptococcus parasanguinis & Firmicutes & 0 & $4,83 \%$ \\
\hline Fibrobacter succinogenes & Fibrobacteres & 0 & $4,14 \%$ \\
\hline Streptococcus sp. M143 & Firmicutes & 0 & $4,14 \%$ \\
\hline Campylobacter concisus & Proteobacteria & 0 & $4,14 \%$ \\
\hline Streptococcus sanguinis & Firmicutes & 0 & $2,76 \%$ \\
\hline Streptococcus sp. M334 & Firmicutes & 0 & $2,76 \%$ \\
\hline Prevotella melaninogenica & Bacteroidetes & 0 & $2,07 \%$ \\
\hline Capnocytophaga ochracea & Bacteroidetes & 0 & $2,07 \%$ \\
\hline Streptococcus vestibularis & Firmicutes & 0 & $2,07 \%$ \\
\hline Veillonella parvula & Firmicutes & 0 & $2,07 \%$ \\
\hline Rothia mucilaginosa & Actinobacteria & 0 & $2,07 \%$ \\
\hline Corynebacterium efficiens & Actinobacteria & 0 & $1,38 \%$ \\
\hline Corynebacterium matruchotii & Actinobacteria & 0 & $1,38 \%$ \\
\hline Granulicatella adiacens & Firmicutes & 0 & $1,38 \%$ \\
\hline Streptococcus gordonii & Firmicutes & 0 & $1,38 \%$ \\
\hline Streptococcus salivarius & Firmicutes & 0 & $1,38 \%$ \\
\hline Leptotrichia buccalis & Fusobacteria & 0 & $1,38 \%$ \\
\hline Actinomyces sp. oral taxon 171 & Actinobacteria & 0 & $0,69 \%$ \\
\hline Thermomonospora curvata & Actinobacteria & 0 & $0,69 \%$ \\
\hline Capnocytophaga gingivalis & Bacteroidetes & 0 & $0,69 \%$ \\
\hline Gemella morbillorum & Firmicutes & 0 & $0,69 \%$ \\
\hline Streptococcus pyogenes & Firmicutes & 0 & $0,69 \%$ \\
\hline Streptococcus sp. C300 & Firmicutes & 0 & $0,69 \%$ \\
\hline Streptococcus sp. oral taxon 071 & Firmicutes & 0 & $0,69 \%$ \\
\hline Streptococcus thermophilus & Firmicutes & 0 & $0,69 \%$ \\
\hline Parvimonas micra & Firmicutes & 0 & $0,69 \%$ \\
\hline Eubacterium saburreum & Firmicutes & 0 & $0,69 \%$ \\
\hline Veillonella sp. 3_1_44 & Firmicutes & 0 & $0,69 \%$ \\
\hline Veillonella sp. oral taxon 158 & Firmicutes & 0 & $0,69 \%$ \\
\hline Fusobacterium sp. 1_1_41FAA & Fusobacteria & 0 & $0,69 \%$ \\
\hline Fusobacterium sp. 3_1_27 & Fusobacteria & 0 & $0,69 \%$ \\
\hline Fusobacterium sp. 7_1 & Fusobacteria & 0 & $0,69 \%$ \\
\hline Lautropia mirabilis & Proteobacteria & 0 & $0,69 \%$ \\
\hline Delftia acidovorans & Proteobacteria & 0 & $0,69 \%$ \\
\hline Simonsiella muelleri & Bacteroidetes & 0 & $0,69 \%$ \\
\hline Treponema vincentii & Spirochaetes & 0 & $0,69 \%$ \\
\hline
\end{tabular}


On the species level, 18 bacterial species were detected in the healthy tissue, 35 species were unique for the OPMD lesions and eight bacterial species were detected both in healthy oral mucosa and the OPMD biopsy samples (Fig. 3b). Metagenome sequencing showed markedly increased bacterial diversity in the OPMD tissue samples. Analysis of bacterial species detected 2.38 fold increasing in diversity of the OPMD lesions (Fig. 4, Table 3).

Based on the data of metagenome sequencing, the relative abundance of Streptococcus sp. did not show significant difference between the healthy tissue and the OPMD lesions: it was $61.82 \%$ in healthy tissue and $58.62 \%$ in OPMD, respectively (Fig. 5a) However, the relative abundance of the Streptococcus mitis was found to be dramatically decreased in OPMD: it was only $18.62 \%$, compared to $32.73 \%$ in the healthy tissue (Fig. 5b). Moreover, examining all Streptococcus species their prevalence in the OPMD lesions was determined to be more diverse compared to the healthy tissue (Table $3)$.

According to the results of metagenome sequencing, the ratio of Fusobacterium nucleatum was higher in the OPMD lesions $(5.52 \%)$, compared to the healthy tissue $(3.64 \%)$ (Fig. 6). Relative abundance of Fusobacterium nucleatum $(5.52 \%)$ and Streptococcus oralis $(4.14 \%)$ bacteria were identified in a larger portion among the overlapping bacteria in OPMD while 3.64 and $1.82 \%$ were observed in healthy tissue, respectively). The abundances of the species Gemella haemolysans (1.38\%), and the above mentioned $S$. mitis $(18.62 \%)$ decreased markedly in OPMD as compared to the healthy tissue $(7.27 \%$ and $32.73 \%$ ) (Table 3 ).
35 different bacterial species has been found in the OPMD lesions which has not been presented in the healthy tissue, e.g., Streptococcus parasanguinis, Fibrobacter succinogenes, Campylobacter concisus, Streptococcus sanguinis, Rothia mucilaginosa, Prevotella melaninogenica, Capnocytophaga ochracea, Streptococcus vestibularis, Veillonella parvula, Corynebacterium efficiens, Corynebacterium matruchotii, Granulicatella adiacens, Streptococcus gordonii, Streptococcus salivarius, Leptotrichia buccalis, Thermomonospora curvata, Capnocytophaga gingivalis, Streptococcus pyogenes Streptococcus thermophiles, Parvimonas micra, Eubacterium saburreum, Lautropia mirabilis, Delftia acidovorans, Simonsiella muelleri, Treponema vincentii, etc., in descending order of the abundances.

As $F$. nucleatum was implicated in carcinogenesis in several cancers [38], we verified the results of metagenome sequencing by $F$. nucleatum-specific qPCR. As Fig. 7 shows, the number of bacteria (CFUs) is significantly higher $(P<0.0001)$ in samples taken from OPMD lesions than in those from the healthy mucosa. This difference is remarkable, even when one considers the high inter-individual variability of the samples.

\section{Discussion}

It was suggested that OPMD was frequently associated with alcohol and tobacco consumption, and affected mainly males in their fifties, but the practice shows that
Fig. 5 Ratio of Streptococci in the mucosal lesion a The ratio of Streptococci to all oral bacteria was not significantly different in the healthy tissue compared to the OPMD lesion. b The ratio of Streptococcus mitis dramatically decreased in the OPMD, as compared to the healthy tissue a

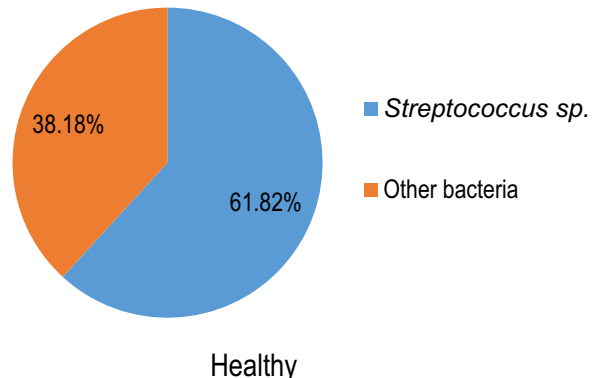

b

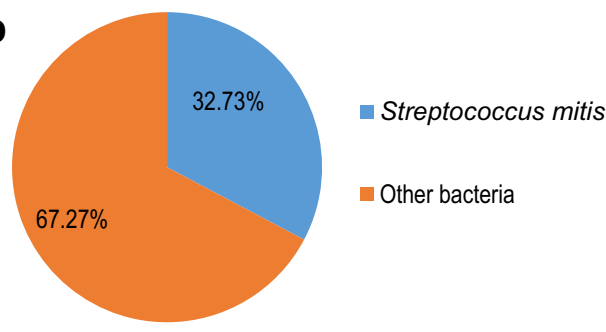

Healthy

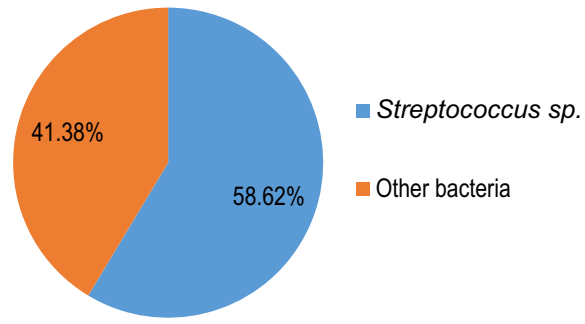

OPMD

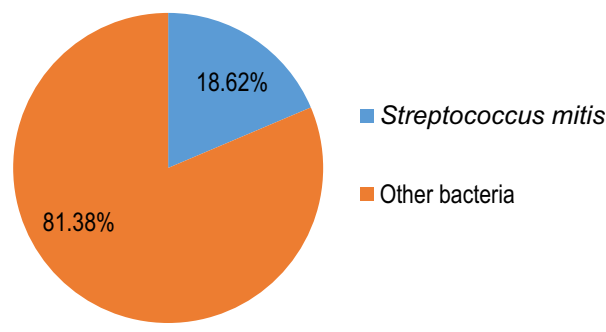

OPMD 
Fig. 6 Fusobacterium nucleatum in the mucosal lesion

The ratio of Fusobacterium nucleatum was markedly increased in the OPMD lesions, as compared to the healthy tissue detected by metagenome sequencing

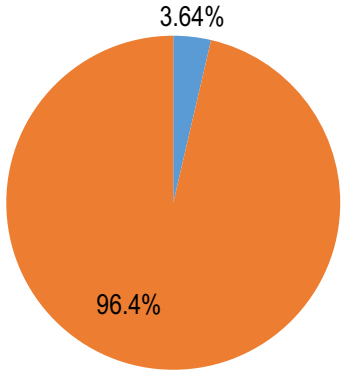

Healthy
- Fusobacterium nucleatum

Other bacteria

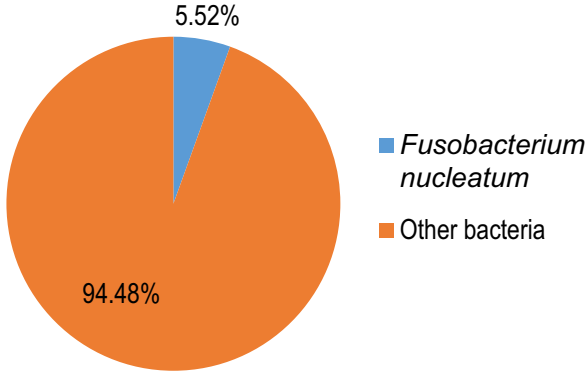

OPMD a much wider variety of patients are affected [1, 3]. Accordingly, we included a wide range of patients in our sample, without strict limitations on age or sex.

In our study we compared the microbiome and microbiota of healthy and the OPMD tissues of oral cavity.

The association of Fusobacterium nucleatum with oral carcinoma was documented [38, 39]. Moreover, it was suggested that distinct subspecies of $F$. nucleatum, such as $F$. nucleatum subsp. polymorphum and $F$. nucleatum subsp. vincentii, may play an etiopathogenetic role in oral carcinogenesis [40]. A study also described the presence of $F$. nucleatum in desquamative gingivitis [41]. However, our report shows the first time the presence of Fusobacterium nucleatum in lesion biopsies of OPMD patients.

The finding that $F$. nucleatum is present in OPMD may be exploited to develop a novel therapeutic strategy of distinct oral disorders such as oral lichen planus. In addition, one may speculate that a targeted antibiotic therapy could be beneficial in preventing the development of oral cancer in a subset of OPMD patients.

We observed that the relative abundance of Streptococcus mitis decreased dramatically in the pathological niche. Since Streptococci are characteristic components of the oral flora, the quantitative analysis of these bacteria is indispensable for the understanding of pathological processes. Streptococci comprise of a wide

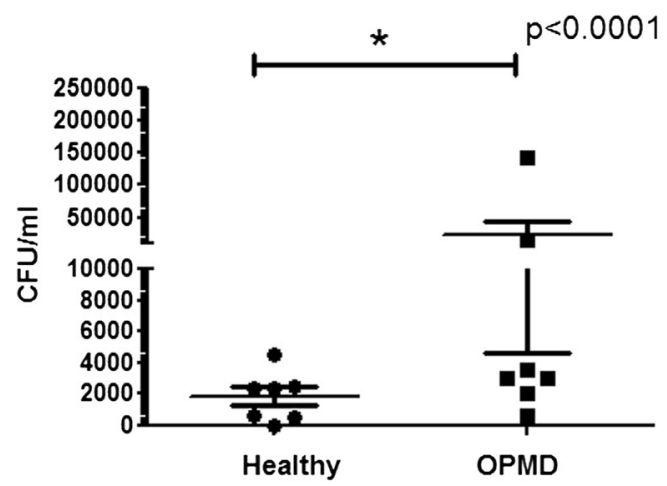

Fig. 7 Fusobacterium nucleatum-specific PCR of the healthy tissue and the OPMD OPMD showed significantly higher colonization by $F$. nucleatum compared to healthy mucosa variety of bacterial species that interact with other members of the oral microbiome. It was suggested that $S$. mitis is involved in the maintenance of a healthy oral flora by affecting adhesion and biofilm formation by periodontal pathogens to [42-44].

Therefore, it is an interesting and important finding that the relative abundance of $S$. mitis in the OPMD lesions decreased to nearly the half of the healthy area. This observation may possibly be exploited for therapeutic purposes: similarly to the reconditioning of vaginal Lactobacillus balance [45], the restitution of $S$. mitis niche in OPMD could also have beneficial effects [46].

Taken together, we presented evidence for the alteration of microbiome and microbiota of OPMD patients. We detected an increased bacterial diversity in the OPMD lesions compared to the healthy oral mucosa. In addition, decreased relative abundance of $S$. mitis and an increased relative abundance of $F$. nucleatum may play a role in the transition of OPMDs to oral cancer.

Although our study is not suitable to answer the "Chicken or the Egg" problem in all aspects, but we have found that the bacterial colonization of mucosa has already altered in OPMD tissues. These observations may form the basis of novel therapeutic approaches preventing oral carcinogenesis in a subset of patients with OPMD.

Acknowledgements This work was in part supported by the National Research, Development and Innovation Fund of Hungary, financed under the NKFI-6-K funding scheme (11493 project), GINOP-2.3.2-15-201600015, János Bolyai Research Scholarship of the Hungarian Academy of Sciences and GINOP 2.3.2-15-2016-00011.

\section{Compliance with Ethical Standards}

Conflict of Interest No potential conflict of interest was reported by the authors.

Ethical Approval The study protocol conformed to the Declaration of Helsinki in all respects and was approved by the Institutional Ethics Committee of the University of Szeged (No. 3161). 


\section{References}

1. Morse DE, Psoter WJ, Cleveland D et al (2007) Smoking and drinking in relation to oral cancer and oral epithelial dysplasia. Cancer Causes Control 18(9):919-929

2. Jepsen SA, Closmann JJ (2008) The insidious nature and presentation of oral squamous cell carcinoma in the low-risk population. Gen Dent 56(1):78-82

3. Llewellyn CD, Linklater K, Bell J et al (2003) Squamous cell carcinoma of the oral cavity in patients aged 45 years and under: a descriptive analysis of 116 cases diagnosed in the South East of England from 1990 to 1997. Oral Oncol 39(2):106-114

4. Nagy KN, Sonkodi I, Szöke I et al (1998) The microflora associated with human oral carcinomas. Oral Oncol 34(4):304-308

5. Sarode SC, Sarode GS, Tupkari JV (2012) Oral potentially malignant disorders: precising the definition. Oral Oncol 48(9):759-760

6. van der Waal I (2009) Potentially malignant disorders of the oral and oropharyngeal mucosa; terminology, classification and present concepts of management. Oral Oncol 45(4-5):317-323

7. Sonkodi I (2009) Oral and Maxilofacial medicine. Semmelweis Publisher, Budapest

8. Silverman S Jr, Gorsky M (1997) Proliferative verrucous leukoplakia. A follow-up study of 54 cases. Oral Surg Oral Med Oral Pathol Oral Radiol Endod 84(2):154-157

9. Fehér E, Gáll T, Murvai M et al (2009) Investigation of the occurrence of torque tenovirus in malignant and potentially malignant disorders associated with human papillomavirus. J Med Virol 81(11):1975-1981

10. Fehér E, Kardos G, Gáll T et al (2011) Comparison of diversity of torque teno virus 1 in different mucosal tissues and disorders. Acta Microbiol Immunol Hung 58(4):319-337

11. Kis A, Fehér E, Gáll T et al (2009) Epstein-Barr virus prevalence in oral squamous cell cancer and in potentially malignant oral disorders in an eastern Hungarian population. Eur J Oral Sci 117(5):536-540

12. Wu L, Feng J, Shi L et al (2013) Candidal infection in oral leukoplakia: a clinicopathologic study of 396 patients from eastern China. Ann Diagn Pathol 17(1):37-40

13. Hebbar PB, Pai A, D S. (2013) Mycological and histological associations of Candida in oral mucosal lesions. J Oral Sci 55(2):157-160

14. Dilhari A, Weerasekera MM, Siriwardhana A et al (2016) Candida infection in oral leukoplakia: an unperceived public health problem. Acta Odontol Scand 74(7):565-569

15. Gainza-Cirauqui ML, Nieminen MT, Novak Frazer L et al (2013) Production of carcinogenic acetaldehyde by Candida albicans from patients with potentially malignant oral mucosal disorders. J Oral Pathol Med. 42(3):243-249

16. Bakri MM, Cannon RD, Holmes AR et al (2014) Detection of Candida albicans ADH1 and ADH2 mRNAs in human archival oral biopsy samples. J Oral Pathol Med 43(9):704-710

17. Kazanowska-Dygdała M, Duś I, Radwan-Oczko M (2016) The presence of helicobacter pylori in oral cavities of patientsn with leukoplakia and oral lichenplanus. J Appl Oral Sci 24(1):18-23

18. Mizuki H, Kawamura T, Nagasawa D (2015) In situ immunohistochemical detection of intracellular mycoplasma salivarium in the epithelial cells of oral leukoplakia. J Oral Pathol Med. 44(2):134-144

19. Meisel P, Holtfreter B, Biffar R et al (2012) Association of periodontitis with the risk of oral leukoplakia. Oral Oncol 48(9):859-863

20. Hu X, Zhang Q, Hua $\mathrm{H}$ et al (2016) Changes in the salivary microbiota of oral leukoplakia and oral cancer. Oral Oncol 56:e6-e8

21. Hernandez BY, Zhu X, Goodman MT et al (2017) Betel nut chewing, oral premalignant lesions, and the oral microbiome. PLoS One 12(2):e0172196

22. Srinivas K, Aravinda K, Ratnakar P et al (2011) Oral lichen planus - Review on etiopathogenesis. Natl J Maxillofac Surg 2(1):15-16
23. Payeras MR, Cherubini K, Figueiredo MA et al (2013) Oral lichen planus: focus on etiopathogenesis. Arch Oral Biol 58(9):10571069

24. Georgakopoulou EA, Achtari MD, Achtaris M et al (2012) Oral lichen planus as a preneoplastic inflammatory model. J Biomed Biotechnol 2012:759626

25. Roopashree MR, Gondhalekar RV, Shashikanth MC et al (2010) Pathogenesis of oral lichen planus-a review. J Oral Pathol Med. 39(10):729-734

26. Gorouhi F, Davari P, Fazel N (2014) Cutaneous and mucosal lichen planus: a comprehensive review of clinical subtypes, risk factors, diagnosis, and prognosis. ScientificWorld Journal 2014:742826

27. Bäckman K, Jontell M (2007) Microbial-associated oral lichenoid reactions. Oral Dis 13(4):402-406

28. Taghavi Zenouz A, Mehdipour M, Jafari Heydarlou M et al (2010) Relationship between Lichen Planus and Helicobacter pylori Infection. J Dent Res Dent Clin Dent Prospects 4(1):17-20

29. Attia EA, Abdel Fattah NS, Abdella HM (2010) Upper gastrointestinal findings and detection of helicobacter pylori in patients with oral lichen planus. Clin Exp Dermatol 35(4):355-360

30. Pourshahidi S, Fakhri F, Ebrahimi H et al (2012) Lack of association between helicobacter pylori infection and oral lichen planus. Asian Pac J Cancer Prev 13(5):1745-1747

31. Izol B, Karabulut AA, Biyikoglu I et al (2010) Investigation of upper gastrointestinal tract involvement and $\mathrm{H}$. Pylori presence in lichen planus: a case-controlled study with endoscopic and histopathological findings. Int J Dermatol 49(10):1121-1126

32. Hulimavu SR, Mohanty L, Tondikulam NV et al (2014) No evidence for Helicobacter pylori in oral lichen planus. J Oral Pathol Med. 43(8):576-578

33. Boorghani M, Gholizadeh N, Taghavi Zenouz A et al (2010) Oral lichen planus: clinical features, etiology, treatment and management; a review of literature. J Dent Res Dent Clin Dent Prospects. 4(1):3-9

34. Ertugrul AS, Arslan U, Dursun R et al (2013) Periodontopathogen profile of healthy and oral lichen planus patients with gingivitis or periodontitis. Int J Oral Sci 5(2):92-97

35. El-Naggar AK, Chan JKC, Takata T, Grandis JR, Slootweg PJ (2017) Tumours of the oral cavity and mobile tongue In: The fourth edition of the head and neck World Health Organization blue book: editors' perspectives. Hum Pathol 66:10-12

36. Sharma PK, Capalash N, Kaur J (2007) An improved method for single step purification of metagenomic DNA. Mol Biotechnol 36(1):61-63

37. Periasamy S, Chalmers NI, Du-Thumm L et al (2009) Fusobacterium nucleatum ATCC 10953 requires Actinomyces naeslundii ATCC 43146 for growth on saliva in a three-species community that includes Streptococcus oralis 34. Appl Environ Microbiol 75(10):3250-3257

38. Rubinstein MR, Wang X, Liu W et al (2013) Fusobacterium nucleatum promotes colorectal carcinogenesis by modulating Ecadherin $/ \beta$-catenin signaling via its FadA adhesion. Cell Host Microbe 14(2):195-206

39. Schmidt BL, Kuczynski J, Bhattacharya A et al (2014) Changes in abundance of oral microbiota associated with oral cancer. PLoS One 9(6):e98741

40. Al-Hebshi NN, Nasher AT, Maryoud MY et al (2017) Inflammatory bacteriome featuring fusobacterium nucleatum and Pseudomonas aeruginosa identified in association with oral squamous cell carcinoma. Sci Rep 7(1):1834

41. Arduino PG, Romano F, Sasia D et al (2017) Subgingival microbiota in white patients with Desquamative gingivitis: a cross-sectional study. J Periodontol 88(7):643-650

42. Kreth J, Merritt J, Qi F (2009) Bacterial and host interactions of oral streptococci. DNA Cell Biol 28(8):397-403 
43. Van Hoogmoed CG, Geertsema-Doornbusch GI, Teughels W et al (2008) Reduction of periodontal pathogens adhesion by antagonistic strains. Oral Microbial Immunol 23(1):43-48

44. Standar K, Kreikemeyer B, Redanz S et al (2010) Setup of an in vitro test system for basic studies on biofilm behavior of mixedspecies cultures with dental and periodontal pathogens. PLoS One 5(10):e13135
45. Otsuki K, Imai N (2017) Effects of lactoferrin in 6 patients with refractory bacterial vaginosis. Biochem Cell Biol 95(1):31-33

46. Klarić M, Mandić V, Lovrić S, e al. (2017) Antimicrobial efficacy of probiotic-containing toothpastes: an in vitro evaluation. Med Glas (Zenica) 14(1):16-24 
II 


\title{
Binary mixture of Satureja hortensis and Origanum vulgare subsp. hirtum essential oils: in vivo therapeutic efficiency against Helicobacter pylori infection
}

\author{
Maria Harmati $^{1}$ | Edina Gyukity-Sebestyen ${ }^{1}$ | Gabriella Dobra ${ }^{1}$ | Gabriella Terhes ${ }^{2}$ | \\ Edit Urban $^{2}$ | Gabor Decsi ${ }^{3}$ | Neda Mimica-Dukić ${ }^{4}$ | Marija Lesjak ${ }^{4}$ | Nataša Simin ${ }^{4}$ | \\ Bernadett Pap ${ }^{5}$ | Istvan B. Nemeth ${ }^{6}$ | Krisztina Buzas ${ }^{1,3}$
}

${ }^{1}$ Laboratory of Microscopic Image Analysis and Machine Learning, Institute of Biochemistry, Biological Research Centre, Hungarian Academy of Sciences, Szeged, Hungary

${ }^{2}$ Institute of Clinical Microbiology, Albert Szent-Györgyi Medical and Pharmaceutical Center, Faculty of Medicine, University of Szeged, Szeged, Hungary

${ }^{3}$ Department of Oral Biology and Experimental Dental Research, Faculty of Dentistry, University of Szeged, Szeged, Hungary

${ }^{4}$ Department of Chemistry, Biochemistry and Environmental Protection, Faculty of Sciences, University of Novi Sad, Novi Sad, Serbia

${ }^{5}$ Laboratory of Microbial Genomics, Institute of Biochemistry, Biological Research Centre, Hungarian Academy of Sciences, Szeged, Hungary

${ }^{6}$ Department of Dermatology and Allergology, University of Szeged, Szeged, Hungary

\section{Correspondence}

Krisztina Buzas, Laboratory of Microscopic Image Analysis and Machine Learning, Institute of Biochemistry, Biological Research Centre, Hungarian Academy of Sciences, Temesvari krt. 62., H-6726, Szeged, Hungary.

Email:kr.buzas@gmail.com

\begin{abstract}
Background: Helicobacter pylori can cause many gastrointestinal and also extragastrointestinal disorders and is a major risk factor for gastric carcinoma and MALT Iymphoma. Currently, numerous antibiotic-based therapies are available; however, these therapies have numerous drawbacks, mainly due to increasing prevalence of antibiotic resistant strains. Thus, there is an urgent need to develop novel therapeutic agents against $H$. pylori infections.

Materials and Methods: In this study, the anti-H. pylori activity of 2:1 mixture of Satureja hortensis and Origanum vulgare subsp. hirtum essential oils (2MIX) was investigated in vivo. After screening in vitro cytotoxicity of $2 \mathrm{MIX}$ on mammalian cell lines, the therapeutic efficiency was studied in a mouse model, where changes in H. pylori colonization were detected by PCR and histology of gastric samples. The immune reaction of mice was tested based on cytokine and chemokine production, and the in vivo toxicity of $2 \mathrm{MIX}$ was also investigated by measuring ALT and AST enzyme activities and Сyp3a11 and HO-1 mRNA levels in livers of mice.

Results: 2 MIX had not shown in vitro cytotoxicity against cell lines, only the highest concentration caused significant decrease in their survival rates. In the in vivo experiments, 2 MIX successfully eradicated the pathogen in $70 \%$ of the mice. We could not detect toxicity or altered cytokine and chemokine balance after in vivo treatments in mice.

Conclusions: These results show that $2 \mathrm{MIX}$ is effective in reducing H. pylori colonization suggesting that this essential oil mixture has great potential as a new, effective, and safe therapeutic agent against $H$. pylori.
\end{abstract}

KEYWORDS

essential oils, Helicobacter pylori, phytotherapy

\section{1 | INTRODUCTION}

Helicobacter pylori is a gram-negative, spiral-shaped, microaerophilic bacterium with an ability to colonize the acidic environment of the stomach. This pathogen infects more than half of the world's human population, and it is considered to be the major cause of chronic gastritis, peptic ulcer disease, gastric cancer, and mucosa-associated lymphoid tissue (MALT) lymphoma. ${ }^{1-4}$ Furthermore, H.pylori infection is reported to be associated not only with gastrointestinal diseases, but also with many extra-gastrointestinal disorders, such as hematological, cardiovascular, neurological, metabolic, and skin diseases. $^{5-7}$ 
The survival capabilities of H. pylori within the stomach make it difficult to eradicate, and effective treatment requires multidrug regimens. Most of them include two antibiotics (usually selected from clarithromycin, metronidazole, amoxicillin, and tetracycline) combined with a proton-pump inhibitor and/or a bismuth compound. ${ }^{8-12}$ These current therapies are generally effective, but can fail due to the increasing prevalence of antibiotic resistance or lack of patient compliance; in addition, they can cause adverse side effects. Thus, there is an urgent need to develop novel therapeutic agents against H. pylori.

Aromatic plants are regarded as a promising source of new and highly potent antibacterial agents. In the last 15 years, several research groups have investigated essential oils from different plants for their in vitro anti-H. pylori activity and presented encouraging results. ${ }^{13,14}$ Still, only a few studies reported their in vivo efficiency, ${ }^{15,16}$ while none of them tested essential oil mixtures, what authors of this study consider very promising due to low ability of $\mathrm{H}$. pylori to develop resistance to a great number of terpenoid structures.

In our previous study, nine aromatic plant species, belonging to three families (Lamiaceae, Apiaceae, and Asteraceae) were selected for testing in vitro anti-H. pylori activity. Firstly, the essential oils of these plants were examined one by one, and then, the most effective essential oils were tested in a combinatory experimental set-up. Finally, the 2:1 mixture of Satureja hortensis (summer savory) and Origanum vulgare subsp. hirtum (Greek oregano) showed the highest inhibitory effect against $H$. pylori growth, probably due to the synergistic effects of the terpenoid compounds. ${ }^{17}$ Based on these results, in this study, this 2:1 mixture (2MIX) was used for in vitro cytotoxicity and in vivo therapeutic examinations to confirm its anti-H. pylori activity in vivo. This research was carried out with ultimate aim which is to support further investigations toward clinical studies and introduction of essential oil therapy as additional therapy to antibiotics for H. pylori patients.

\section{2 | METHODS}

\section{1 | Plant material and essential oil preparation}

Essential oils were prepared as described previously. ${ }^{17}$ Briefly, the plant material of $S$. hortensis and $O$. vulgare subsp. hirtum was purchased from the organic farm "Farago," Orom, Vojvodina, Serbia. The voucher specimens were prepared and identified by Goran Anačkov, PhD, and deposited at the Herbarium of the Department of Biology and Ecology, University of Novi Sad Faculty of Sciences, Serbia (2-1542 and 2-1539 for S. hortensis and O. vulgare subsp. hirtum, respectively). The plant material was collected in a flowering stage and subsequently air-dried and finely grounded. The essential oils were isolated by hydrodistillation according to the recommended procedure by Ph. EUR. IV. ${ }^{18}$ The 2:1 mixture (v/v) of $S$. hortensis and $O$. vulgare subsp. hirtum essential oil (2MIX) was prepared. The obtained $2 \mathrm{MIX}$ was stored at $-20^{\circ} \mathrm{C}$ prior to further experiments.

\section{2 | In vitro cell viability assay}

Four different cell lines were chosen to study the cytotoxicity of 2MIX: HepG2 human hepatocarcinoma, THP-1 human monocyte, RAW 264.7 mouse macrophage, and B16F1 mouse melanoma cell lines. All of them were maintained in a $37^{\circ} \mathrm{C}$ humidified incubator with $5 \% \mathrm{CO}_{2}$, in the appropriate cell-specific medium which consisted of EMEM supplemented with 10\% FBS, 1\% NEAA, 1\% L-glu for HepG2, RPMI-1640 with 10\% FBS, $0.05 \mathrm{mmol} / \mathrm{L}$ 2-mercaptoethanol for THP-1, DMEM with 10\% FBS, 1\% NEAA, 1\% VIT, 1\% L-glu, 0.01\% Na-pyruvate for B16F1, and DMEM F-12 with 10\% FBS for RAW 264.7.

Cells were cultured in $2 \mathrm{MIX}$-containing or control medium (medium without $2 \mathrm{MIX}$ ) for 48 hours. Based on previous in vitro inhibitory effect of the $2 \mathrm{MIX}$ on $\mathrm{H}$. pylori growth, ${ }^{17}$ dosages of treatments were $0.01 \%, 0.001 \%$, and $0.0001 \%$. Oils were incorporated to the media by sonication. Morphological changes in the cells were observed microscopically, and the survival rates were evaluated by trypan blue exclusion 24 and 48 hours after treatments.

\section{3 | Bacterial strain and culture conditions}

Metronidazole resistant, mouse-adapted H.pylori SS1 strain ${ }^{19}$ used here was kindly provided by Dr. Eliette Touati (Unité de Pathogenése de Helicobacter Département de Microbiologie, Pasteur Institute, Paris, France) and grown on brain heart infusion broth medium (BHIB; HiMedia, Mumbai, India) supplemented with $2.5 \%$ yeast extract (Torlak, Belgrade, Serbia), 5\% horse serum (Gibco, Gaithersburg, MD, USA), and 2\% IsoVitaleX (BD, Le Pont de Claix, France). The bacterium cultures were maintained under microaerobic conditions $\left(5 \% \mathrm{O}_{2}, 10 \% \mathrm{CO}_{2}, 85 \% \mathrm{~N}_{2}\right)$ and moist atmosphere. The purity of the culture was checked regularly.

\section{4 | Animal model}

Specific-pathogen-free six- to eight-week-old female Balb/c mice were obtained from Charles River (Germany). They were maintained $a b$ three to five animals per cage, with wood pellet bedding, on commercial diet and water ad libitum.

Infections with H. pylori SS1 strain were established three times over a five-day period by administration via feeding needle of $100 \mu \mathrm{L}$ bacterium suspension in BHIB $\left(10^{9} \mathrm{CFU} / \mathrm{mL}\right)$. Negative control mice remained uninfected. One week later, some animals were sacrificed to verify the success of infection by PCR before treatments. At the same time, infected mice were randomly divided into three groups. First of them remained untreated (positive control), second was treated by $2 \mathrm{MIX}(43.75 \mu \mathrm{L} / \mathrm{kg}$ body weight) and third with sunflower oil, the diluent of $2 \mathrm{MIX}$ (Table 1). Treatments were carried out in $50 \mu \mathrm{L}$ volume, three times over a five-day period by feeding needle. Another week later, blood samples were collected via cardiac puncture and all of the animals were sacrificed under anesthesia. The entire stomach of each mouse was removed for assessment of $H$. pylori colonization, and liver samples were collected for toxicological investigations.

All animal experiments were performed in accordance with national (1998. XXVIII; 40/2013) and European (2010/63/EU) animal ethics 
TABLE 1 Treatment schedules for groups of mice

\begin{tabular}{|lrll}
\hline Group name & n & $\begin{array}{l}\text { Helicobacter pylori } \\
\text { infection }\end{array}$ & Treatment \\
\hline Negative control & 5 & - & - \\
\hline 2MIX-treated & 12 & + & 2 MIX \\
\hline Sunflower oil-treated & 5 & + & Sunflower oil \\
\hline Positive control & 5 & + & - \\
\hline
\end{tabular}

guidelines. The experimental protocols were approved by the Animal Experimentation and Ethics Committee of the Biological Research Centre of the Hungarian Academy of Sciences and the Hungarian National Animal Experimentation and Ethics Board (clearance number: XVI./03521/2011.)

\section{5 | Assessment of H. pylori colonization levels}

\subsection{1 | PCR}

After the gastric samples were homogenized in $100 \mu \mathrm{L}$ lysis buffer (ZR Kit), DNA was isolated using ZR Fecal DNA MiniPrep ${ }^{\text {TM }}$ kit (Zymo Research Corporation, Irvine, CA, USA) according to the manufacturer's protocol.

A 229 -bp region of the $16 \mathrm{~S}$ rRNA sequence is $100 \%$ homologous in most $\mathrm{H}$. pylori strains. ${ }^{20}$ In the PCR analysis, specific primers for a part of this region, $5^{\prime}$-TCG GAA TCA CTG GGC GTA A- $3^{\prime 20}$ and 5'-GCG TCA GTA ATG TTC CAG CA-3' (designed for this study), were used. PCR was performed at $95^{\circ} \mathrm{C}$ for 1 minute, followed by 35 cycles at $95^{\circ} \mathrm{C}$ for 30 seconds, $60^{\circ} \mathrm{C}$ for 30 seconds, $72^{\circ} \mathrm{C}$ for 30 seconds, and a final step at $72^{\circ} \mathrm{C}$ for 4 minutes. The 202 -bp PCR products were separated on $2 \%(\mathrm{w} / \mathrm{v})$ agarose gels, stained with ethidium bromide and visualized using a UV transilluminator.

\subsection{2 | Histology}

Gastric samples were fixed in 4\% buffered formaldehyde for 24 hours, embedded in paraffin blockers and cut into $4 \mu \mathrm{m}$ serial sections (Leica RM2245). Each sample was autostained (Leica ST5020) by routine Giemsa for highlighting H. pylori bacteria. Slides were archived by virtual microscopy using slide scanner (3DHistech, Budapest, Hungary).

\section{6 | Preparation of serum samples and cytokines analysis}

Collected blood samples were allowed to clot for 30 minutes at room temperature and then overnight at $4^{\circ} \mathrm{C}$. Serum samples were collected by centrifugation at $850 \times \mathrm{g}$ for 5 minutes and stored at $-80^{\circ} \mathrm{C}$ until analysis.

Serum cytokines were analyzed by Proteome Profiler Mouse Cytokine Array Panel (R\&D Systems, Minneapolis, MN, USA) according to the manufacturer's instructions. A known volume of $250 \mu \mathrm{L}$ of pooled serum samples was applied for each membrane, and the chemiluminescent signal was detected using Odyssey imaging system (LI-COR).

\subsection{Analysis of in vivo toxicity}

\subsection{1 | Liver AST and ALT enzyme activities}

The liver aspartate aminotransferase (AST) and alanine aminotransferase (ALT) enzyme activities were determined using the ALT (GPT) and AST (GOT) stable liquid reagent kits (Clinichem, Budapest, Hungary), according to the manufacturer's instructions.

In brief, the AST assay is based on two reactions. The first one is catalyzed by AST and includes two substrates: L-aspartate and a-ketoglutarate. Second reaction consists of transformation of oxalacetate, released in the first reaction, by malate dehydrogenase with the help of NADH coenzyme, both contained in the reagent. The oxidoreductive process of $\mathrm{NADH} / \mathrm{NAD}^{+}$is indicated by a decrease in absorbance at $340 \mathrm{~nm}$ which correlates with liver AST activity.

Similarly, in ALT assay, ALT firstly catalyzes the transformation of L-alanin and a-ketoglutarate. The pyruvate released in before mentioned reaction is transformed by lactate dehydrogenase in the presence of $\mathrm{NADH}$, both contained in the reagent. NADH/NAD oxidoreductive process shows a decrease in absorbance at $340 \mathrm{~nm}$ which correlates with liver ALT activity.

Before carrying out assays, liver tissue homogenates were prepared in TRIS buffer ( $\mathrm{pH} 7.5$ ) and supernatants were used for further ALT and AST activities estimations. Afterward, the total protein content in each homogenate was determined. The enzyme activities were expressed according to NADH calibration curve measured on $340 \mathrm{~nm}$ using NADH solutions in TRIS buffer $(\mathrm{pH}$ 7.5). Samples were prepared according to manufacturer's instructions and after an one-minute incubation at $37^{\circ} \mathrm{C}$, changes in absorbance at $340 \mathrm{~nm}$ were measured each 30 seconds $(\triangle \mathrm{A} / \mathrm{min})$ during two minutes. Consequently, all results were expressed as $\mathrm{NADH} \mathrm{mmol} / \mathrm{min} \mathrm{mg}$ proteins.

\subsection{2 | RT-PCR analysis of heme oxygenase 1 and cytochrome P450 mRNA}

Liver RNA was extracted with TRIzol ${ }^{\circledR}$ (Life Technologies, Waltham, MA, USA) according to the manufacturer's instructions. Total RNA ( $1 \mu \mathrm{g})$ was reverse transcribed using the High Capacity cDNA Reverse Transcription Kit (Life Technologies), according to the manufacturer's instructions. Real-time PCR reactions were performed using Agilent Technologies Mx3005P cycler using glyceraldehyde-3-phosphate dehydrogenase (GAPDH) as internal standard. Each reaction was performed in duplicate and contained 10 pmol of specific primers (heme oxygenase 1 (HO-1) fwd 5'-GAA CCC AGT CTA TGC CCC AC-3', rev 5'-GGC GTG CAA GGG ATG ATT TC-3'; cytochrome P450, family 3, subfamily a, polypeptide 11 (Cyp3a11) fwd 5'-AGG AGG ATC ACA CAC ACA GTT G-3', rev 5'-CCC AGG TTT CCA GTG AGA GAG-3'; GAPDH fwd 5'-ACT CTT CCA CCT TCG ATG CC-3', rev 5'-GGC CTC TCT TGC TCA GTG TC$3^{\prime}$ ), 2× Brilliant II SYBR Green qPCR Master Mix (Agilent Technologies, Santa Clara, CA, USA), $0.375 \mu \mathrm{L}$ of provided reference dye (diluted 1:500 in nuclease-free $\mathrm{H}_{2} \mathrm{O}$ ) and $4 \mu \mathrm{L}$ of cDNA in a $25-\mu \mathrm{L}$ reaction. Samples without cDNA were included as negative controls. Cycle threshold (Ct) values were obtained for each gene of interest and the GAPDH internal 

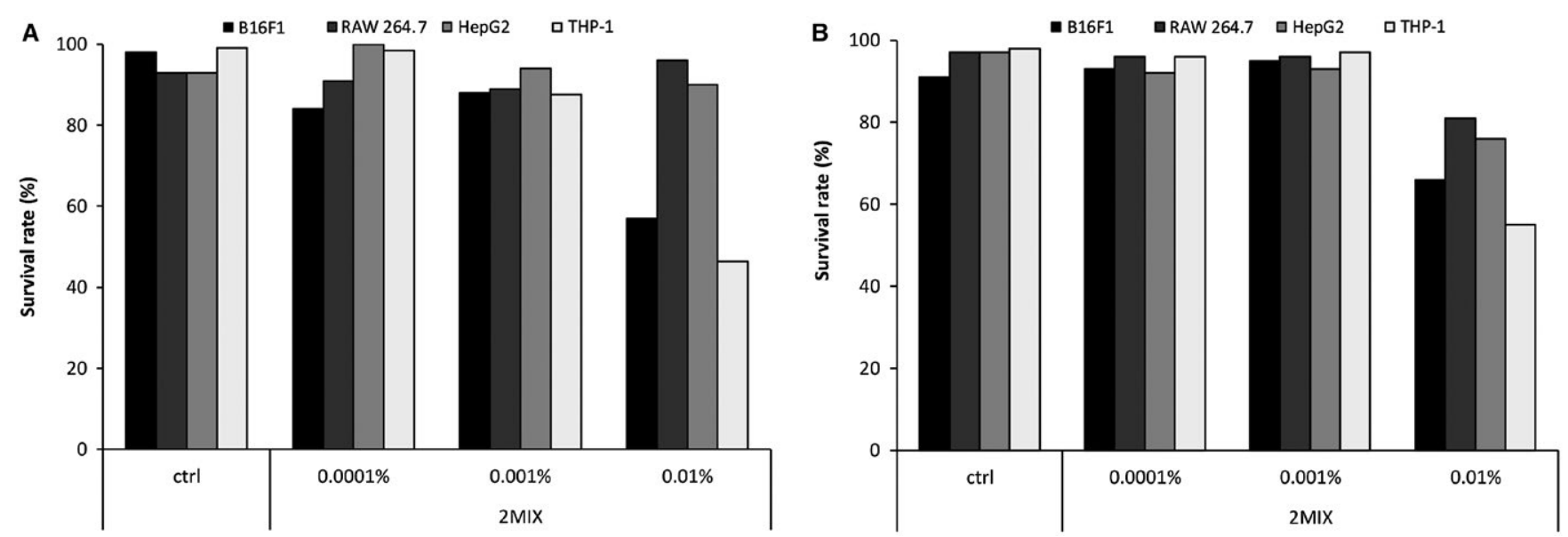

FIGURE 1 Effect of $2 \mathrm{MIX}$ on viability of the mammalian cell lines. (A) Twenty-four hours after treatments: Only the highest concentration of $2 \mathrm{MIX}$ caused significant decrease in survival rates of B16F1 $(P=.0001)$ and THP-1 $(P<.0001)$ cells, but not RAW 264.7 and HepG2. (B) Fortyeight hours after treatments: Also only the highest concentration of $2 \mathrm{MIX}$ caused significant decrease in survival rates of the cells: $\mathrm{B} 16 \mathrm{~F} 1$ $(P=.03)$, RAW $264.7(P=.012)$, HepG2 $(P=.005)$, THP-1 $(P<.0001)$

standard. Gene expression was normalized to GAPDH and represented as $\Delta \mathrm{Ct}$ values. For each sample, the mean of the $\Delta \mathrm{Ct}$ values was calculated. Relative gene expression was normalized to $1.0(100 \%)$ of controls.

\section{3 | RESULTS}

\section{1 | Cytotoxicity against mammalian cell lines}

As in the preliminary in vitro studies, $2 \mathrm{MIX}$ showed high antibacterial activity against $\mathrm{H}$. pylori, ${ }^{17}$ we aimed to test cytotoxicity of $2 \mathrm{MIX}$ against four different mammalian cell lines (RAW 264.7, B16F1, HepG2, and THP-1) before the in vivo experiments. Cells were treated with cell line-specific medium alone or medium containing $2 \mathrm{MIX}$ at three different concentrations (0.01\%, 0.001\%, or 0.0001\%). After 24 and 48 hours, the viability of the cells was determined by trypan blue exclusion assay. 2 MIX had not caused significant decrease in survival rates of the cells, except at the highest concentration (Fig. 1).

\section{2 | In vivo antibacterial effect}

After verifying low levels of cytotoxicity, we tested the effect of $2 \mathrm{MIX}$ on $\mathrm{H}$. pylori colonization in mice. On the first week of the in vivo experiments, mice were infected with the mouse-adopted H.pylori SS1 strain. On the third week, establishment of infection was confirmed by PCR and histology and the infected mice were treated by 2 MIX or sunflower oil (diluent of the therapeutic agent). An additional group of infected mice remained untreated as a positive control, and we had an uninfected, untreated negative control group (Table 1). On the fifth week, mice were sacrificed and the colonization of $H$. pylori was tested by PCR and histology in gastric samples.

We could verify the long-term H. pylori infection of mice (Fig. 2). In the 2 MIX-treated group, only $30 \%$ of the animals remained positive for the presence of the pathogen, confirmed by the PCR analysis (Fig. 3). As expected, sunflower oil had no effect on H. pylori

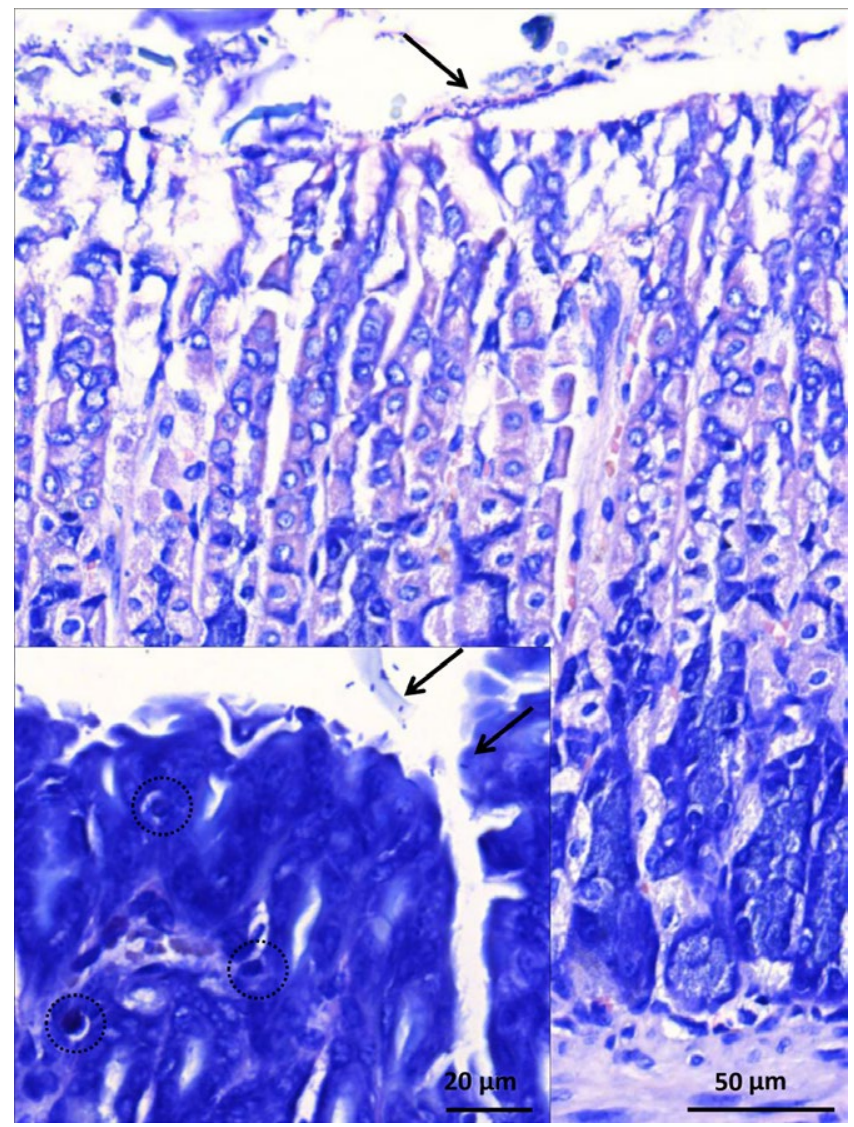

FIGURE 2 Long-term H. pylori infection of positive control (infected, untreated) mice. Giemsa-stained gastric specimens five weeks after infection. Arrows show H. pylori foveolar colonization in gastric fundus sample (Giemsa staining; OM 200x), and the intra-epithelial mononuclear lymphoid elements of the mucosa are noted (circles)

infection. In the negative control group, absence of the bacteria was also confirmed. These in vivo results suggest promising anti-H. pylori therapeutic potential of 2MIX. 


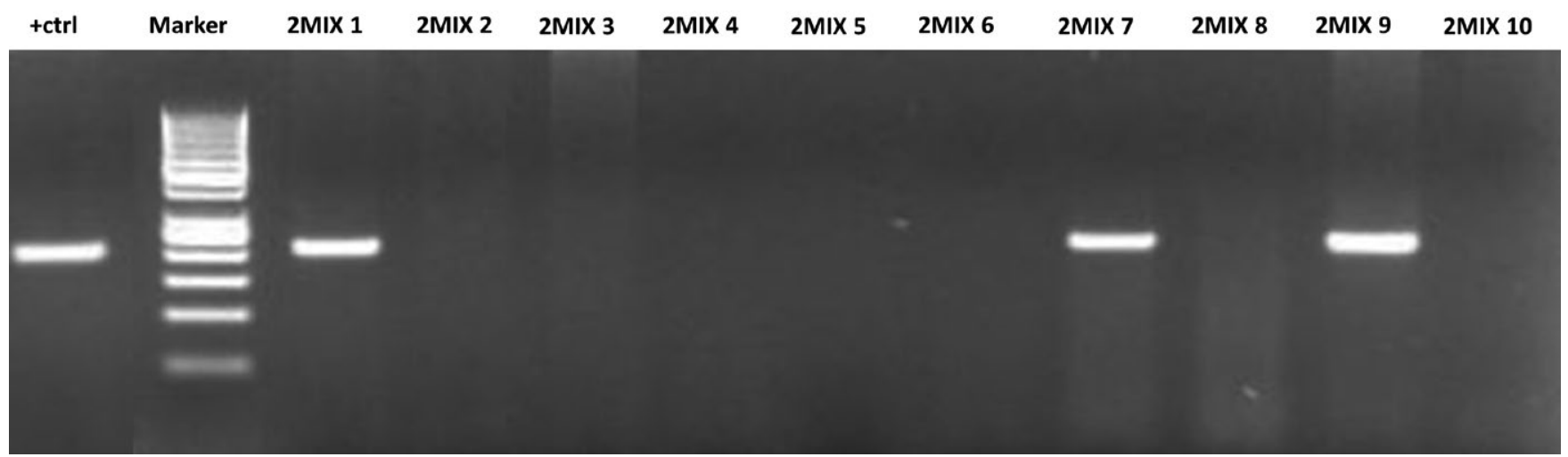

FIGURE 3 Changes in the level of H. pylori colonization after treatments. Agarose gel electrophoresis of PCR products showed elimination of the bacteria from gastric samples of the $2 \mathrm{MIX}$-treated mice

\subsection{Effect of treatments on immune response}

After confirmation of the anti-H. pylori activity of $2 \mathrm{MIX}$ in vivo, we attempted to clarify the effect of $2 \mathrm{MIX}$ as therapeutic agent on immune response. A set of 40 chemokines and cytokines was analyzed by Proteome Profiler Array in serum of mice. We could not detect any significant changes in the cytokine profile among all groups (Fig. 4), and this profile was the same with the manufacturer's reference cytokine profile of mouse serum. These results suggest that $2 \mathrm{MIX}$ do not have any immunomodulatory or allergic effects in vivo.

\section{4 | In vivo toxicity of 2 MIX}

We could not observe significant differences among groups in the case of the liver AST and ALT enzyme activities because NADH levels were comparable between all groups (Table 2).

Although $\mathrm{H}$. pylori infections and the subsequent treatments had not changed the expression of Cyp3a11, the level of HO-1 mRNA increased after the $H$. pylori infections and decreased again after the successful eradication of the pathogen in the $2 \mathrm{MIX}$ treated group (Table 3). In conclusion, 2MIX was not toxic in vivo, but only $\mathrm{HO}-1$ mRNA showed increased expression due to the $H$. pylori infection.

\section{4 | DISCUSSIONS}

This study primarily aimed to investigate the in vivo anti- $H$. pylori effect of the 2:1 mixture of $S$. hortensis and $O$. vulgare subsp. hirtum essential oils ( $2 \mathrm{MIX})$, as our previous in vitro results demonstrated that this essential oil mixture would be a highly effective therapeutic agent against $H$. pylori. ${ }^{17}$

In our previous in vitro experiments, the high complexity of $2 \mathrm{MIX}$ resulted in fourfold higher anti-H. pylori activity than the individual oils and twofold higher activity than other essential oil combinations, probably due to the increased synergistic effect between components of $S$. hortensis and $O$. vulgare subsp. hirtum essential oils. ${ }^{17}$ This result came as no surprise as other recent in vitro studies also demonstrated synergism of essential oil or ethanol plant extract mixtures in proven antibacterial, antifungal, or antioxidant activities. ${ }^{21-23}$
Namely, essential oils are complex mixtures of numerous volatile terpenoid compounds, from which all components and their unique combinations greatly contribute to their antibacterial effects. It is believed that terpenoids could have an impact on cell penetration, as well as lipophilic or hydrophilic attraction and fixation on cell walls and membranes. ${ }^{24}$ Due to synergism between terpenoids, whole essential oils have greater antibacterial activity than the major components alone. $^{25-27}$

Contrary, essential oil components may show not only synergistic, but also antagonistic effects when their combination has lower activity than the sum of their individual effects. ${ }^{28}$ The overall activity of a mixture probably depends on several factors such as the ratio of active components, the characteristics of target microorganisms, and the microenvironmental factors (e.g., $\mathrm{pH}$ or temperature). For example, Lambert et al. ${ }^{29}$ observed synergism between carvacrol and thymol investigating their antibacterial effect against Pseudomonas aeruginosa and Staphylococcus aureus, while Falsafi et al. ${ }^{26}$ demonstrated antagonism between this two components studying anti-H.pylori activity. Therefore, a mixture of essential oils is not necessarily superior to the individual oils. ${ }^{30}$ Consequently, combining components or whole essential oils requires detailed considerations and preliminary experiments.

After the proven outstanding antibacterial effect of $2 \mathrm{MIX}$ in vitro, its therapeutic efficiency was investigated in the present in vivo study in which Balb/c mice were stably colonized by H. pylori SS1 strain. After oral treatments with essential oils, $70 \%$ of the animals in the 2MIX-treated group had been completely cured. What is more this therapy did not cause any side effect or immune response in mice, which make this result even more significant. Namely, absence of side effects additionally supports further in vivo and potential clinical studies which could promote essential oils as new additional therapy, aside with antibiotics, for patient suffering from $H$. pylori infection.

Facts which also support safe anti-H. pylori usage of 2 MIX in humans are that investigated oils are extensively included in the diet and folk medicine since ancient time. Namely, people worldwide traditionally use Satureja and Origanum species as herbal teas and spices, ${ }^{31,32}$ while previous studies reported the potential of oregano essential oil to preserve food. ${ }^{33-35}$ In traditional medicine, Greek oregano has been 


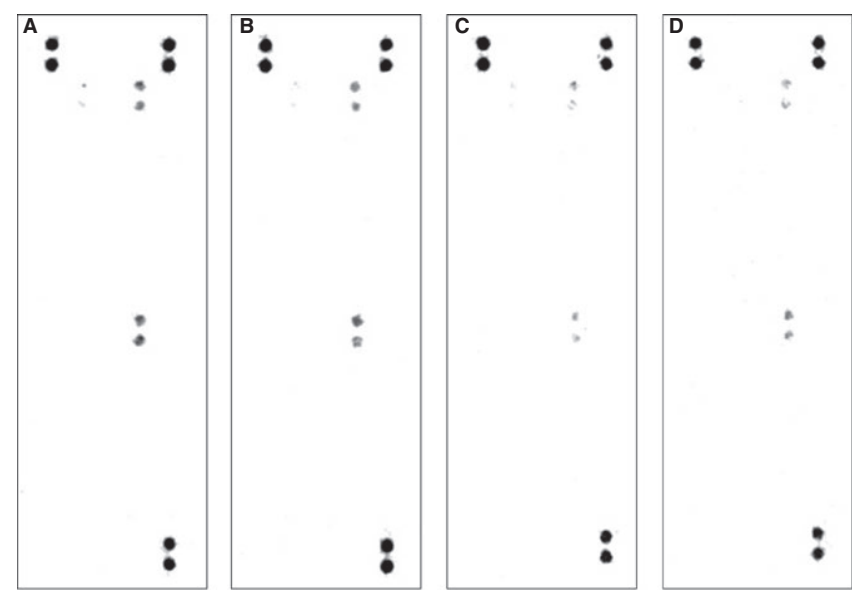

FIGURE 4 Cytokine profiles of pooled serum samples in each group. (A) -ctrl, (B) +ctrl, (C) sunflower oil-treated group, (D) 2MIXtreated group. Two weeks after treatments, the production of cytokines has not changed according to the mouse proteome profiler array

used to treat respiratory disorders, dyspepsia, painful menstruation, rheumatoid arthritis, scrofulosis, and urinary tract disorders. ${ }^{32}$ Also, summer savory is a traditional remedy for treatment of various symptoms, such as muscle pain, stomach and intestinal disorders-nausea, indigestion, and diarrhea, as well as various infectious diseases. ${ }^{31,36,37}$

However, there are no in vivo toxicology studies of Greek oregano essential oils on humans. Namely, the only in vivo study on this oil was carried out by Hollenbach et al. ${ }^{38}$ which showed that oregano essential oil acted directly on sexual organs, reducing their weight, decreasing testosterone levels, and causing tissue injury of the testes in rats. Still, these effects were observed after the treatment with much higher dose of essential oil (i.e., $27 \% \mathrm{v} / \mathrm{v}$ ) than those applied in our study.

Concerning summer savory, there is no clinical evidence for dose limitation. Also, contraindications, side effects, and potential drug interactions of this oil in humans have not been identified yet. ${ }^{39}$ However, in single animal study, it is determined that the $\mathrm{LD}_{50}$ of summer savory essential oil is $1.37 \mathrm{~g} / \mathrm{kg}$ for oral administration, which is about 31 times higher than concentration applied in our study. ${ }^{40}$

Results of this study are of great value especially because the in vitro anti-H. pylori activity of different essential oils was previously demonstrated in several cases, while studies on their in vivo efficiency are scarce. Specifically, Ohno et al. ${ }^{15}$ reported that the lemon grass oil significantly reduced the bacterial colonization of mice, but only $10 \%$ of the animals were completely cured. In an other study carried out by Bergonzelli et al., only a subgroup of mice responded to the examined carrot seed essential oil treatment. The therapy eradicated the pathogen from $20 \%$ to $30 \%$ of the mice, but the bacterial colonization did not significantly decrease in rest of the oiltreated animals. ${ }^{16}$ Our studies indicate that $2 \mathrm{MIX}$ is highly effective in vivo against $H$. pylori, as $70 \%$ of the mice had been cured after 2MIX-treatment.

In conclusion, these results suggest that the 2:1 mixture of S. hortensis and $O$. vulgare subsp. hirtum essential oils may have potential as a new, effective, and safe therapeutic agent against $H$. pylori infections. Synergistic effects between their compounds increase the antibacterial efficiency and make it more effective than the individual oils. Above all, these results support further more complex investigation toward introduction of $2 \mathrm{MIX}$ into official clinical anti-H. pylori therapy.

TAB LE 2 Effect of orally administered 2MIX on liver AST and ALT enzyme activities

\begin{tabular}{|c|c|c|c|c|}
\hline \multirow[b]{2}{*}{ Group } & \multicolumn{2}{|l|}{ AST activity } & \multicolumn{2}{|l|}{ ALT activity } \\
\hline & $\mathrm{NADH}$ (mmol/min mg) & $P$ value & $\mathrm{NADH}$ (mmol/min mg) & $P$ value \\
\hline Positive control & $0.095 \pm 0.035$ & - & $0.059 \pm 0.003$ & - \\
\hline 2MIX-treated & $0.118 \pm 0.059$ & .291 & $0.071 \pm 0.024$ & .137 \\
\hline Sunflower oil-treated & $0.110 \pm 0.014$ & .335 & $0.071 \pm 0.014$ & .142 \\
\hline Negative control & $0.114 \pm 0.061$ & .741 & $0.062 \pm 0.012$ & .595 \\
\hline
\end{tabular}

AST, aspartate aminotransferase; ALT, alanine aminotransferase.

Given $P$ values denote difference from the positive control group.

TABLE 3 Effect of orally administered 2MIX on liver Cyp3a11 and HO-1 gene expression in mice

\begin{tabular}{|c|c|c|c|c|}
\hline Group & \multicolumn{2}{|l|}{ Сур3а11/GAPDH } & \multicolumn{2}{|l|}{ HO-1/GAPDH } \\
\hline 2MIX-treated & $0.66 \pm 0.25$ & .370 & $0.52 \pm 0.14$ & $<.001$ \\
\hline Sunflower oil-treated & $0.75 \pm 0.28$ & .961 & $0.75 \pm 0.06$ & .038 \\
\hline
\end{tabular}

Cyp3a11, cytochrome P450, family 3, subfamily a, polypeptide 11; HO-1, heme oxygenase 1; GAPDH, glyceraldehyde-3-phosphate dehydrogenase. Given $P$ values denote difference from the positive control group. 


\section{ACKNOWLEDGEMENTS}

This research was funded by project HELICO (project ID: HUSRB/1203/2.1.4) in the scope of Hungary-Serbia IPA Cross-border Co-operation program, Hungarian Scientific Research Fund (OTKA K) 112493 and GINOP-2.3.2-15-2016-00001.

\section{REFERENCES}

1. Kuipers EJ, Thijs JC, Festen HP. The prevalence of Helicobacter pylori in peptic ulcer disease. Aliment Pharmacol Ther. 1995;9(Suppl 2):59-69.

2. Conteduca V, Sansonno D, Lauletta G, Russi S, Ingravallo G, Dammacco F. H. pylori infection and gastric cancer: state of the art (review). Int J Oncol. 2013;42:5-18.

3. Kim SS, Ruiz VE, Carroll JD, Moss SF. Helicobacter pylori in the pathogenesis of gastric cancer and gastric lymphoma. Cancer Lett. 2011;305:228-238.

4. Watari J, Chen N, Amenta PS, et al. Helicobacter pylori associated chronic gastritis, clinical syndromes, precancerous lesions, and pathogenesis of gastric cancer development. World J Gastroenterol. 2014;20:5461-5473.

5. Leontiadis GI, Sharma VK, Howden CW. Non-gastrointestinal tract associations of Helicobacter pylori infection. Arch Intern Med. 1999;159:925-940.

6. Wong F, Rayner-Hartley E, Byrne MF. Extraintestinal manifestations of Helicobacter pylori: a concise review. World J Gastroenterol. 2014;20:11950-11961.

7. Franceschi F, Tortora A, Gasbarrini G, Gasbarrini A. Helicobacter pylori and extragastric diseases. Helicobacter. 2014;19(Suppl 1):52-58.

8. McColl KEL. Helicobacter pylori infection. N Engl J Med. 2010;362:1597-1604.

9. Chey WD, Wong BC. Practice Parameters Committee of the American College of Gastroenterology. American College of Gastroenterology guideline on the management of Helicobacter pylori infection. Am J Gastroenterol. 2007;102:1808-1825.

10. Malfertheiner P, Megraud F, O'Morain CA, et al. Management of Helicobacter pylori infection-the Maastricht IV/Florence Consensus Report. Gut. 2012;61:646-664.

11. Chuah S-K, Tsay F-W, Hsu P-I, Wu D-C. A new look at anti-Helicobacter pylori therapy. World J Gastroenterol. 2011;17:3971-3975.

12. Delchier JC, Malfertheiner P, Thieroff-Ekerdt R. Use of a combination formulation of bismuth, metronidazole and tetracycline with omeprazole as a rescue therapy for eradication of Helicobacter pylori. Aliment Pharmacol Ther. 2014;40:171-177.

13. Reichling J, Schnitzler P, Suschke U, Saller R. Essential oils of aromatic plants with antibacterial, antifungal, antiviral, and cytotoxic properties-an overview. Forsch Komplementmed. 2009;16:79-90.

14. Bonifácio B, Dos Santos Ramos M, da Silva P, Bauab T. Antimicrobial activity of natural products against Helicobacter pylori: a review. Ann Clin Microbiol Antimicrob. 2014;13:54.

15. Ohno T, Kita M, Yamaoka Y, et al. Antimicrobial activity of essential oils against Helicobacter pylori. Helicobacter. 2003;8:207-215.

16. Bergonzelli GE, Donnicola D, Porta N, Corthésy-Theulaz IE. Essential oils as components of a diet-based approach to management of Helicobacter infection. Antimicrob Agents Chemother. 2003;47:3240-3246.

17. Lesjak M, Simin N, Orcic D, et al. Binary and tertiary mixtures of Satureja hortensis and Origanum vulgare essential oils as potent antimicrobial agents against Helicobacter pylori. Phytother Res. 2016;30:476-484.

18. European Pharmacopeia. Council of Europe: Strasbourg Cedex, France. 4th edn; 2002:183-184.

19. Lee A, O'Rourke J, De Ungria MC, Robertson B, Daskalopoulos G, Dixon MF. A standardized mouse model of Helicobacter pylori infection: introducing the Sydney strain. Gastroenterology. 1997;112:1386-1397.
20. Liu H, Rahman A, Semino-Mora C, Doi SQ, Dubois A. Specific and sensitive detection of $H$. pylori in biological specimens by real-time RT-PCR and in situ hybridization. PLoS ONE. 2008;3:e2689.

21. Bag A, Chattopadhyay RR. Evaluation of synergistic antibacterial and antioxidant efficacy of essential oils of spices and herbs in combination. PLoS ONE. 2015;10:e0131321.

22. Fratini F, Casella S, Leonardi M, et al. Antibacterial activity of essential oils, their blends and mixtures of their main constituents against some strains supporting livestock mastitis. Fitoterapia. 2014;96:1-7.

23. Baljeet SY, Simmy G, Ritika Y, Roshanlal Y. Antimicrobial activity of individual and combined extracts of selected spices against some pathogenic and food spoilage microorganisms. Int Food Res J. 2015;22:2594-2600.

24. Bakkali F, Averbeck S, Averbeck D, Idaomar M. Biological effects of essential oils-a review. Food Chem Toxicol. 2008;46:446-475.

25. de Mendonça Rocha PM, Rodilla JM, Díez D, et al. Synergistic antibacterial activity of the essential oil of aguaribay (Schinus molle L.). Molecules. 2012;17:12023-12036.

26. Falsafi T, Moradi P, Mahboubi M, Rahimi E, Momtaz H, Hamedi B. Chemical composition and anti-Helicobacter pylori effect of Satureja bachtiarica Bunge essential oil. Phytomedicine. 2015;22:173-177.

27. Trinh NT, Dumas E, Thanh ML, et al. Effect of a Vietnamese Cinnamomum cassia essential oil and its major component trans-cinnamaldehyde on the cell viability, membrane integrity, membrane fluidity, and proton motive force of Listeria innocua. Can J Microbiol. 2015;61:263-271.

28. Burt S. Essential oils: their antibacterial properties and potential applications in foods-a review. Int J Food Microbiol. 2004;94:223-253.

29. Lambert RJ, Skandamis PN, Coote PJ, Nychas GJ. A study of the minimum inhibitory concentration and mode of action of oregano essential oil, thymol and carvacrol. J Appl Microbiol. 2001;91:453-462.

30. Karbach J, Ebenezer S, Warnke PH, Behrens E, Al-Nawas B. Antimicrobial effect of Australian antibacterial essential oils as alternative to common antiseptic solutions against clinically relevant oral pathogens. Clin Lab. 2015;61:61-68.

31. MomtazS,Abdollahi M. An update on pharmacology of Satureja species: from antioxidant, antimicrobial, antidiabetes and anti-hyperlipidemic to reproductive stimulation. Int J Pharmacol. 2010;6:454-461.

32. Gruenwald J, Brendler T, Jaenicke C. PDR for Herbal Medicines. Montvale: Medical Economic Co., Inc.; 2000.

33. Chouliara E, Karatapanis A, Savvaidis IN, Kontominas MG. Combined effect of oregano essential oil and modified atmosphere packaging on shelf-life extension of fresh chicken breast meat, stored at $4^{\circ} \mathrm{C}$. Food Microbiol. 2007;24:607-617.

34. Giatrakou V, Kykkidou S, Papavergou A, Kontominas MG, Savvaidis IN. Potential of oregano essential oil and MAP to extend the shelf life of fresh swordfish: a comparative study with ice storage. J Food Sci. 2008;73:M167-M173.

35. Atrea I, Papavergou A, Amvrosiadis I, Savvaidis IN. Combined effect of vacuum-packaging and oregano essential oil on the shelf-life of Mediterranean octopus (Octopus vulgaris) from the Aegean Sea stored at $4^{\circ} \mathrm{C}$. Food Microbiol. 2009;26:166-172.

36. Bezic N, Samanic I, DunKic V, Besendorfer V, Puizina J. Essential oil composition and internal transcribed spacer (ITS) sequence variability of four South-Croatian Satureja apecies (Lamiaceae). Molecules. 2009;14:925-938.

37. Hajhashemi V, Sadraei H, Ghannadi AR, Mohseni M. Antispasmodic and anti-diarrheal effect of Satureja hortensis L. essential oil. J Ethnopharmacol. 2000;71:187-192.

38. Hollenbach CB, Bing RS, Stedile R, et al. Reproductive toxicity assessment of Origanum vulgare essential oil on Male Wistar Rats. Acta Sci Vet. 2015;43:1295.

39. Gardner Z, McGuffin M. American Herbal Products Association's Botanical Safety Handbook, 2nd edn. Boca Raton: CRC Press; 2013.

40. Opdyke DIJ. Monographs on Fragrance Row Materials. New York: Pergamons; 1979. 


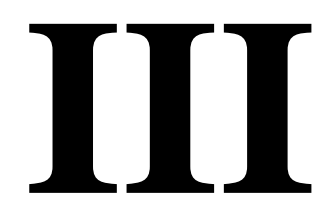




\title{
Infectious Agents Associated with Head and Neck Carcinomas
}

\author{
Andrea Hettmann*, Anett Demcsák*, Gábor Decsi, \\ Ádám Bach, Dóra Pálinkó, László Rovó, Katalin Nagy, \\ Mária Takács, and Janos Minarovits
}

\begin{abstract}
In addition to traditional risk factors such as smoking habits and alcohol consumption, certain microbes also play an important role in the generation of head and neck carcinomas. Infection with high-risk human papillomavirus types is strongly associated with the development of oropharyngeal carcinoma, and Epstein-Barr virus appears to be indispensable for the development of non-keratinizing squamous cell carcinoma of the nasopharynx. Other viruses including torque teno virus and hepatitis $C$ virus may act as co-carcinogens, increasing the risk of malignant transformation. A shift in the composition of the oral microbiome was associated with the development of oral squamous cell carcinoma, although the causal or casual role of oral bacteria remains to be clarified. Conversion of ethanol to acetaldehyde, a mutagenic compound, by members of the oral microflora as well as by fungi including Candida albicans and others is a potential mechanism that may increase oral cancer risk. In addition, distinct Candida spp. also produce NBMA (N-nitrosobenzylmethylamine), a potent carcinogen. Inflammatory processes elicited by microbes may also facilitate tumorigenesis in the head and neck region.
\end{abstract}

*Author contributed equally with all other contributors.

A. Hettmann* and M. Takács

Division of Virology, National Center for Epidemiology, Albert F. ut 2-6, H-1097 Budapest, Hungary

A. Demcsák* and J. Minarovits $(\bowtie)$

Faculty of Dentistry, Department of Oral Biology and Experimental Dental Research, University of Szeged, Szeged, Hungary

e-mail: minimicrobi@hotmail.com
G. Decsi and K. Nagy

Department of Oral Surgery, University of Szeged, Tisza Lajos krt. 64, H-6720 Szeged, Hungary

Á. Bach, D. Pálinkó, and L. Rovó

Faculty of Medicine, Department of Oto-RhinoLaryngology and Head-Neck Surgery, University of Szeged, Tisza L. krt. 111, H-6725 Szeged, Hungary 


\section{Keywords}

Human papillomavirus - Oropharyngeal carcinoma Epstein-Barr virus • Nasopharyngeal carcinoma $\bullet$ Co-carcinogen $\bullet$ Torque teno virus $\bullet$ Hepatitis $\mathrm{C}$ virus $\bullet$ Oral microbiome $\bullet$ Mutagenic compound $\bullet$ Candida albicans

1

\section{Introduction}

Head and neck cancer is a broad term that encompasses cancers arising in the head and neck region. They may originate from the mucosal lining of the oral cavity, nasal cavity, paranasal sinuses, oropharynx, nasopharynx, hypopharynx, and larynx, or begin in the lip or in the salivary glands (Bose et al. 2013). Most of these malignancies are head and neck squamous cell carcinomas (HNSCCs). Head and neck cancer is the sixth most common cancer type worldwide with approximately 650000 new cases annually (Ferlay et al. 2010). Despite the advancements of treatment methods, including chemotherapy, radiotherapy, and surgery, the 5-year survival rate of HNSCC patients improved only modestly in the past decades: it is around $50 \%$, mostly due to locoregional recurrences, distant metastases and additional primary tumors (Leemans et al. 2011).

The major risk factors for HNSCC are tobacco use and alcohol consumption and they seem to have a multiplicative combined effect. Genetic polymorphisms in enzymes that metabolize tobacco and alcohol have been linked to an increased risk for HNSCC (Cadoni et al. 2012; Maurya et al. 2014). Smokeless tobacco and chewing of betel quid are also known risk factors for oral cancer (Li et al. 2015; Sand et al. 2014). There is wide geographic variation in the incidence and anatomic distribution of HNSCC worldwide. This variation is predominately attributed to demographic differences in the habits of tobacco use and alcohol consumption. In the western part of the world the incidence of HNSCC has declined, mostly due to the decline of tobacco use. In contrast, oral cancer is the leading type of malignancies among men in high risk countries, such as India, Pakistan, Sri Lanka and Bangladesh, (Joshi et al. 2014). Similarly, the estimated incidence and mortality rate of lip, oral cavity and pharynx carcinomas is high in Central and Eastern European countries (Hungary, Slovakia, Romania), possibly due to traditional risk factors (Iriti and Varoni 2015).

According to the International Agency on Research for Cancer (IARC), in 2008 around two million of the estimated 12.7 million new cancer cases occurring worldwide could be attributed to infections (IARC Working Group 2012; de Martel et al. 2012). In addition to oncogenic viruses (HPV, MCPyV, EBV, HHV-8, HBV, HCV, HTLV-I) and bacteria (Helicobacter pylori) other infectious agents may also contribute to the development of malignant tumors. A series of microbe-induced pathological alterations including mutations, cell cycle modulation, inhibition of DNA repair, epigenetic dysregulation, inflammation and immune system impairment may facilitate tumorigenesis (Alibek et al. 2013).

One of the well-documented virus-cancer relationships is the association of high-risk human papillomavirus (HPV) infection with a subset of HNSCCs located predominantly to the oropharynx. In the US and Western Europe, there was a recent increase in oropharyngeal cancer incidence, compared to other head and neck cancers. This phenomenon was attributed to a higher prevalence of high-risk HPV strains in the oral mucosa (Mehanna et al. 2013; Näsman et al. 2009; Rietbergen et al. 2013).

The nearly ubiquitous Epstein-Barr virus (EBV) plays a major role in the development of a series of neoplasms including undifferentiated nasopharyngeal carcinoma (NPC). NPC is a rare cancer globally, but it is the leading cancer type in distinct high-risk populations, especially in Southern China, indicating that non-viral, genetic and environmental factors also contribute to NPC development (Jia and Qin 2012). Although compared to HPV and EBV the evidence is less direct, recent data also suggested a role for other viral, as well as bacterial and fungal infections in the etiology of head and neck cancer (Sand and Jalouli 2014). 
Carcinogenesis is a multistep process driven by genetic and epigenetic alterations that result typically in the clonal or oligoclonal expansion of cells (Hanahan and Weinberg 2000). Over $90 \%$ of HNSCCs arise from pre-existing potentially malignant lesions or conditions, e.g. oral leukoplakia and oral lichen planus. The treatment of high risk oral premalignancies, however, did not efficiently prevent either their recurrence or the development of oral carcinomas (Braakhuis et al. 2003; Leemans et al. 2011). This phenomenon was attributed to the existence of a "field effect", i.e. genetic alterations predisposing large areas of oral mucosa for tumorigenesis ('field cancerization'). Recurrences and novel new malignant transformations occur preferentially at such 'fields' (Braakhuis et al. 2003; Leemans et al. 2011). It is worthy to note that epigenetic abnormalities characteristic for laryngeal squamous cell carcinomas may also extend to the adjacent normal mucosa, indicating the occurrence of an "epigenetic field of cancerisation" (Paluszczak et al. 2011).

We wish to overview the contribution of infectious agents including viruses, bacteria and fungi to the development of HNSCC. A better understanding of microbe-induced molecular events, including genetic and epigenetic changes, in head and neck cancer development may pave the way for novel therapies and prevention strategies.

\section{Human Papillomavirus}

Human papillomaviruses (HPVs) of the Papillomaviridae family are small, non-enveloped viruses with a double-stranded, circular DNA genome of about 8000 bp. There are 174 completely characterized HPV types, classified on the basis of the capsid protein L1 gene sequences (Bzhalava et al. 2013). The discovery that distinct HPV types were associated with cervical carcinoma was a significant milestone in tumor virology (reviewed by zur Hausen 2009).

HPVs have strict host selectivity for humans. Cutaneous types infect the skin, whereas mucosal types infect nonkeratinized squamous epithelia lining the oral cavity, the esophagus and the vagina. HPVs can be grouped into high and low risk types based on their capacity to induce malignant transformation. High risk types include HPV 16, 18, 31, 33, 34, 35, 39, 45, $51,52,56,58,59,66,68,73$ and 82 ; they were associated with high grade intraepithelial lesions of the cervix and with invasive cancer. In contrast, non-oncogenic or low-risk HPV types, including HPV 6, 11, 40, 42, 43, 44, 54, 61, 72, 81 and 89, were detected in low grade intraepithelial lesions (Woods et al. 2014).

HPV is associated with wide range of diseases from benign warts to invasive cancer. There is strong epidemiological evidence for the involvement of HPV infection in the generation of six non-skin cancer types, including the carcinomas of cervix, penis, vulva, vagina, anus and upper aerodigestive tract (de Martel et al. 2012). The majority of HPV related head and neck cancers are located to the oropharynx, an anatomic region comprised of the soft palate, uvula, tonsils, posterior pharyngeal wall, and the base of the tongue (Gillison et al. 2014). It was suggested that tonsillar crypts may trap the virus and inhibit mechanical clearance. In addition, the monolayer of epithelial cells that lines these crypts could be more susceptible to HPV infection than stratified epithelium (Elrefaey et al. 2014; Klussmann et al. 2001). It is worthy to note that the oropharyngeal SCCs associated with traditional risk factors, i.e. smoking and alcohol consumption, are usually moderately differentiated and show a keratinizing phenotype. In contrast, the majority of HPV-associated head and neck cancers lack significant keratinization and are of basaloid morphology (Westra 2009).

\section{The HPV Genome}

All open reading frames in the $8 \mathrm{~kb}$ HPV genome are located on one of the two DNA strands. Most HPV-infected cells carry circular, episomal HPV DNA molecules, whereas HPV-associated carcinomas harbor viral genomes integrated into the cellular DNA. The HPV genome can be divided into three major regions. Early gene transcription and replication is regulated by the long control region (LCR) that contains promoter and enhancer elements as well as the viral origin of replication. The downstream coding ORFs are 
called early and late genes and their names refer to their location as well as the timing of their transcription. The products of the early genes (E1-E7) are necessary for the genome maintenance and replication. E5, E6 and E7 are the oncoproteins of HPV, although they have their important role in the normal lifecycle of the virus as well. The E2 protein, apart from having a role in genome replication, regulates the transcription of the other early genes. The late genes (L1 and L2) code for the structural proteins, with L1 being the major and L2 the minor capsid protein (Rautava and Syrjänen 2012; Stanley 2012).

\section{$4 \quad$ HPV Epidemiology}

As for the prevalence of HPV in oropharyngeal cancer cases, there are significant differences between various anatomical regions. A recent meta-analysis by Mehanna et al. found $47.7 \%$ overall pooled HPV prevalence in oropharyngeal cancer, whereas $21.8 \%$ of non-oropharyngeal squamous cell carcinomas were HPV positive. HPV prevalence was found to significantly increase over time from $40,5 \%$ before 2000 to $72,2 \%$ after 2005. The overall HPV prevalence of head and neck malignancies differed by geographical region as well: North-America and Europe had the highest prevalence. In the last decades Northern and Western European countries have reported a steep rise in the proportion of HPV associated oropharyngeal cancers (Mehanna et al. 2013; Marur et al. 2010; Näsman et al. 2009; Rietbergen et al. 2013; Louie et al. 2015). However, regions with low prevalence of HPV have reported lower prevalence of HPV DNA in HNSCC patients (López et al. 2014), and similarly low prevalence was found in relatively high-risk regions for HNSCC, in Central-Europe and Latin-America. In these communities traditional risk factors may play a more important role in the development of HNSCCs (Ribeiro et al. 2011; Marur et al. 2010).

HPV can be found in other types of head and neck cancers as well. Isayeva et al. analyzed the prevalence rates of HPV in oral cavity, laryngeal, sinonasal and nasopharyngeal carcinomas. Lower prevalence rates were detected $(20.2 \%, 23.6 \%$, $29.6 \%$ and $31.1 \%$, respectively) compared to oropharyngeal cancers (Isayeva et al. 2012). They also found that the prevalence of HPV in potentially premalignant and premalignant oral lesions is significantly higher than the rate of oral HPV carriers. Interestingly, submucous fibrosis showed the highest HPV prevalence (11/12, $91.7 \%)$. Submucous fibrosis is a potentially malignant condition of the oral cavity and it is linked to chewing of betel quid (Jalouli et al. 2010).

Independently of overall HPV prevalence in HNSCCs, HPV16 was the most abundant HPV type found in these malignancies. HPV16 accounted for $86.7 \%$ of HPV positive oropharyngeal cancer; it was less prevalent, however, in other types of head and neck cancer. A smaller proportion was attributable to HPV18; other high risk types were rarely found in these cancers (Kreimer et al. 2005; Chaturvedi 2012). It was observed that HPV associated oropharyngeal cancers tend to have better prognosis than HPV-positive carcinomas located to other anatomic regions (Lindquist et al. 2007; Ang et al. 2010; El-Mofty 2012, Mellin et al. 2012, Sethi et al. 2012; Ramqvist et al. 2015).

\section{$5 \quad$ Characteristics of Patients with HPV-Associated Head and Neck Cancer}

People diagnosed with HPV related HNSCC tend to be younger ( $<60$ years of age) than those with HNSCC caused by traditional risk factors ( $>60$ years of age) (Marur et al. 2010; Genden et al. 2013). Although the absolute proportion of HNSCC affecting young adults (18-40 years of age) remains low (1-6\%), epidemiological studies have shown a steady rise in the incidence of oropharyngeal and oral cavity cancer in this population (Majchrzak et al. 2014).

Several aspects of sexual behavior are strongly associated with HPV positive HNSCC. These include younger age at first intercourse, total lifetime number of vaginal and oral sex partners and lack of barrier use during sexual intercourses (Gillison et al. 2008; Chaturvedi 2012; Burke et al. 2014). 
The incidence of both HPV-positive and HPV-negative HNSCC are higher in men. The male to female ratio has declined in oral cancer, and now it is about 1.5:1; however in HPV-associated HNSCC the male:female ratio remains 3:1 (Gillison et al. 2012; Warnakulasuriya 2009). This phenomenon can't be explained exclusively by the differences in sexual behavior between the two genders, and suggests some male predisposition to oropharyngeal cancer. It might be attributed to a protective effect of seroconversion in women due to earlier cervical HPV infection (Safaeian et al. 2010), or to the higher oral HPV prevalence among men. The latter is possibly due to a more effective transmission of HPV through oral sex on women versus men (Gillison et al. 2012).

\section{$6 \quad$ HPV Induced Carcinogenesis: Integration of the Viral Genome into the Host Cell DNA}

After initial infection, the HPV genome persists in episomal form within the host cells. Typically, there is no integration of the viral genome into the cellular DNA during productive infection of differentiating epithelial cells. The virus relies on the cellular replication machinery for the replication of its own genome (Lazarczyk et al. 2009). Malignant transformation is associated with high-level expression of viral E6 and E7 oncoproteins. During HPV-initiated carcinogenesis, high-level E6 and E7 expression frequently occurs after the integration of the viral DNA into the host cell genome (Vinokurova et al. 2008). The integration breakpoint is usually within the E2 gene encoding a negative regulator of E6 and E7 transcription. Thus, the integration of the viral genome disrupts the regulatory function of E2 and leads to constitutively active high-level E6 and E7 expression and increased cell proliferation (Parfenov et al. 2014; Williams et al. 2011; Rautava and Syrjänen 2012). The integration event also causes genomic instability by inducing chromosomal rearrangements, DNA amplification and disruption of tumor suppressor genes (Parfenov et al. 2014). In certain neoplasms, however, the HPV DNA can also be found in episomal form, or there is a combination of episomal and integrated form. In HPV16associated oropharyngeal cancer the episomal or combined forms of viral genomes dominate. In cases when HPV remained in episomal form, a higher episomal count and a high viral load was detected (Mellin et al. 2002; Deng et al. 2013; Parfenov et al. 2014; Olthof et al. 2015).

\section{$7 \quad$ HPV Oncoproteins}

Three of the HPV proteins encoded by the early region of the viral genome, E5, E6 and E7 were implicated in carcinogenesis. The E5 gene is frequently lost during integration and a large fraction of HPV-associated tumors do not express E5 protein (reviewed by Venuti et al. 2011). E5 might contribute to oncogenesis, however, in case of HPV genomes that persist as episomes in tumor cells (Venuti et al. 2011). E5 may play a role in immune evasion by reducing the MHC-I level on the cell surface (Stöppler et al. 1996; Campo et al. 2010). In addition, E5 may promote cell growth by enhancing epidermal growth factor receptor-mediated signaling (DiMaio and Mattoon 2001).

In high-risk HPV-associated oropharyngeal carcinoma cells, continuous expression of the E6 and E7 oncoproteins is essential for the maintenance of the transformed phenotype (Rampias et al. 2009). The mechanisms contributing to E6 and E7 mediated oncogenesis are complex. Here we wish to outline only some of the best documented carcinogenetic pathways.

E7 has neither direct DNA binding activity nor enzymatic activity, but it is able to interact with key cellular regulators. E7 binds and induces the degradation of the retinoblastoma protein $(\mathrm{Rb})$, a tumor suppressor protein that regulates the G1-S transition of the cell cycle (reviewed by Rautava and Syrjänen 2012; Boyer et al. 1996). Rb interacts with the E2F family of transcription factors that activate genes indispensable for S-phase entry and progression (McLaughlin-Drubin and Münger 2010). Thus, binding of E7 to Rb results in constitutive expression of $\mathrm{E} 2 \mathrm{~F}$ responsive genes and 
leads to DNA synthesis. Degradation of $\mathrm{pRB}$ induces the upregulation of $\mathrm{p} 16^{\mathrm{INK} 4 \mathrm{~A}}$ (p16) tumor suppressor protein, and the elevated p16 level is used as a diagnostic marker in HPV positive HNSCC (Fakhry et al. 2014). E7, a pleiotropic regulator, also interacts with a series of other cellular proteins involved in the control of cell cycle and affects the gene expression pattern of host cells by binding to key epigenetic regulators (reviewed by Klingelhutz and Roman 2012; Moody and Laimins 2010).

The E6 protein of high-risk HPVs forms complex with, and targets the tumor suppressor protein p53 for proteasomal degradation by recruiting the cellular ubiquitin ligase E6AP. In addition, the E6-p53 interaction interferes with the binding of p53 to DNA and blocks p53 acetylation (McLaughlin-Drubin and Münger 2010). Because HPV-associated oropharyngeal cancers harbor wild type, non-mutated p53 that has a pro-apoptotic activity, it was suggested that disrupting the E6-p53 complex may induce apoptosis in HPV related malignancies (Li and Johnson 2013; Caicedo-Granados et al. 2014).

Genomic instability is an early event in HPV-associated carcinogenesis (Moody and Laimins 2010). In addition to the integration of the HPV genome into the host cell DNA, HPV E6 and E7 may also contribute to the development of chromosomal aberrations, partly by blocking p53, an important factor maintaining the stability of the genome, and partly by inducing centrosome abnormalities (McLaughlinDrubin and Münger 2010).

It is worthy to note that HPV directly inhibits interferon synthesis and signaling via the interaction of E6 and E7 proteins with components of the interferon signaling pathways (reviewed by Stanley 2012). Such a mechanism may facilitate the immune escape of HPV-positive carcinomas.

\section{Epstein-Barr Virus: The First Human Tumor Virus}

Epstein-Barr virus (EBV), a human gammaherpesvirus, is associated with both lymphomas and carcinomas, including Burkitt's lymphoma (BL),
Hodgkin's lymphoma, midline granuloma, posttransplant lymphoproliferative disorders (PTLDs), X-linked lymphoproliferative syndrome, nasopharyngeal carcinoma (NPC), gastric carcinoma, and others (reviewed by Shah and Young 2009; Sugden 2014). In immunosuppressed and immunodeficient patients, EBV-related leiomyosarcomas, i.e. smooth muscle neoplasms may also develop (Dalal et al. 2008). EBV, the first human tumor virus, was discovered in BL cell cultures. Although it was initially considered a purely lymphotropic virus, an in situ hybridization study of anaplastic NPCs revealed the presence EBV DNA in the carcinoma cells, but not in infiltrating lymphocytes, suggesting a role for EBV in the malignant transformation of epithelial cells, too (Epstein et al. 1964; Sugden 2014; Wolf et al. 1973).

\section{$9 \quad$ Estein-Barr Virus: Basic Facts}

Epstein-Barr virus (EBV, also known as human herpesvirus 4, HHV-4) belongs to the genus Lymphocryptovirus within the subfamily Gammaherpesvirinae of the family Herpesviridae. The prototype EBV genome is $172 \mathrm{kbp}$ in length and it is packaged into the virions as a linear DNA molecule. Upon infection of B-lymphoid and epithelial cells, the linear EBV genome undergoes circularization, and latent EBV genomes typically persist as circular episomes attached to the nuclear matrix. Latent EBV episomes co-replicate with the cellular DNA and display a restricted gene expression pattern. In contrast, all viral genes are expressed and a large number of linear EBV genomes are generated upon induction of lytic, productive EBV replication. Depending on the host cell phenotype, latent EBV genomes adopt distinct gene expression patterns (latency types) and the activity of latent viral promoters is regulated by the cellular epigenetic machinery (reviewed by Takacs et al. 2010). In turn, latent, growth-transformation associated EBV proteins affect the host cell transcriptome and epigenome through the interaction with cellular epigenetic regulators (reviewed by Niller et al. 2009). 


\section{EBV and Nasopharymgeal Carcinoma: Epidemiology}

EBV is a ubiquitous herpesvirus spreading among humans most commonly through saliva and other bodily fluids. The majority of the population undergoes inapparent primary infection in early childhood. The virus replicates in oropharyngeal epithelial cells, but also infects B lymphocytes, and latent EBV genomes are carried by resting, memory B cells for life. Primary EBV infection in teenagers or adults causes infectious mononucleosis (IM, also called glandular fever), a self-limiting disease (reviewed by Niller et al. 2007; Sugden 2014).

Nasopharyngeal carcinoma was the first cancer of the head and neck region that was found to be associated with a human virus (Wolf et al. 1973).

There are two major types or classes of nasopharyngeal carcinoma, keratinizing squamous cell carcinoma accounting for $20 \%$ of NPC cases, and non-keratinizing squamous cell carcinoma. Keratinizing squamous cell carcinomas of the nasopharynx occur sporadically throughout the world at a relatively low incidence. They are either EBV-positive or EBV negative, depending on the geographical area (Nicholls et al. 1997). The non-keratinizing type represents $80 \%$ of NPC cases and it is invariably associated with EBV (reviewed by Shah and Young 2009). Although EBV infects human populations all over the world, the incidence of the non-keratinizing type of NPC, including both differentiated and undifferentiated forms carrying latent EBV episomes in almost $100 \%$ of cases, occurs with a high incidence at restricted geographical locations, i.e. it is an endemic tumor in Southeast Asia, Tunisia, and among Alaskan and Greenland Inuit (reviewed by Shah and Young 2009; Niller et al. 2007).

\section{Risk Factors Affecting the Incidence of Nasopharyngeal Carcinoma}

Several genetic risk factors were identified in China, especially in the Guangzhou area, that may contribute to the high NPC incidence. These include an allele encoding a member of
cytochrome-P450 super-family of proteins involved in the activation of carcinogenic compounds, a gene coding for a glutathione S-transferase that contributes to the detoxification of carcinogens, as well as genes located to or near to HLA loci and genes encoding enzymes and regulators of the DNA repair machinery (reviewed by Lung et al. 2014; Niller et al. 2007). It is worthy to note that epigenetic inactivation of cellular genes, mainly by promoter hypermethylation, also plays an important role in the generation of NPC (reviewed by Niller et al. 2014; Li et al. 2011).

Additional risk factors are volatile nitrosamines that may act as initiators of chemical carcinogenesis and phorbol ester-like compounds that may act as promoters, stimulating the proliferation of cells carrying mutated, initiated genomes. Such compounds are present in medical herbal teas or in the diet, including salted fish. Phorbol-ester-like compounds such as diterpene-esters may also induce lytic EBV replication, i.e. reactivation of latent EBV genomes in B cells infiltrating the nasopharyngeal epithelium (Ito et al. 1983; Ho et al. 1978). Increased local EBV load may facilitate the infection of epithelial cells, an early event in NPC development.

\section{NPC: Carcinogenesis}

As briefly outlined above, in addition to EBV infection, genetic factors and environmental carcinogens also play a role and act in concert during the initiation and progression of NPC. Based on these observations, Lo et al. elaborated a collaborative model for NPC tumorigenesis (Lo et al. 2012). They suggested that DNA damage elicited by carcinogens (e.g. nitrosamines from salted fish and preserved food) and local chronic inflammation might cause chromosomal aberrations that could be detected in the dysplastic lesions of the nasopharynx. A typical finding was the deletion of the short arm of chromosome 3 (3p) affecting several tumor suppressor genes. In addition, epigenetic mechanisms such as promoter hypermethylation also contribute to the silencing of tumor suppressor genes already in 
EBV-negative dysplastic lesions. Centrosome abnormalities and the generation of multipolar spindles may also induce genetic instability at this stage. Loss of the p16 tumor suppressor gene may result in the overexpression of cyclin D1 that favours stable latent EBV infection of nasopharyngeal epithelial cells. EBV infection and the clonal proliferation induced by latent EBV proteins and RNAs may accelerate neoplastic development (Lo et al. 2012).

Viral gene expression patterns of NPC typically corresponds to the so called latency type II.: in addition to the nuclear protein EBNA1 (EBV nuclear antigen 1), a variable expression of LMP1 (latent membrane protein 1) and LMP2A (latent membrane protein2A) is observed in NPC (reviewed by Niller et al. 2007). LMP1 is an oncoprotein, its expression in rodent cells results in malignant transformation. The EBV genome encodes non-translated microRNAs as well that act as posttranscriptional regulators of mRNA and protein levels. Hsu et al. observed that miR-BART9 targets the E-cadherin mRNA and promotes migration and metastasis formation by NPC cells (Hsu et al. 2014).

\section{Other Viruses Possibly Associated with Head and Neck Carcinomas and Oral Precancerous Lesions: Torque Teno Virus (TTV) and Hepatitis C Virus (HCV)}

Torque Teno viruses (TTVs) belong to the family of Anelloviridae and have a small, circular, single stranded DNA genome (reviewed by Spandole et al. 2015). Although the first TTV-like sequence was found in the serum of a patient with posttransfusion hepatitis, at present, TTV is not linked to either hepatitis or any other disease as a causative agent (Nishizawa et al. 1997; Okamoto 2009). TTVs are ubiquitous viruses with a nearly $100 \%$ prevalence that establish persistent infection (Saback et al. 1999; Hsieh et al. 1999; Zhong et al. 2001; Ninomiya et al. 2008; Hussain et al. 2012; Vasilyev et al. 2009). Children may be infected by the end of their first year and simultaneous infections may also occur (Ninomiya et al. 2008). TTVs can be found in wide range of tissues and body fluids including liver, bone marrow, lymph nodes, spleen, pancreas, thyroid, lungs, kidneys, PMBCs, saliva, urine, tears, nasal secretion, feces, throat swabs, bile and semen (Spandole et al. 2015). In addition, TTV related sequences were detected in many different human diseases including AIDS, neoplasia, asthma and rheumatoid arthritis (Moen et al. 2002; Thom and Petrik 2007; Pifferi et al. 2005; Saláková et al. 2009; Figueiredo et al. 2007; Suzuki et al. 2014; de Villiers et al. 2007; Gergely et al. 2006). Regarding head and neck cancer, TTV related sequences were found in laryngeal cancer (de Villiers et al. 2002). Furthermore, co-infection with genogroup 1 TTV and HPV was associated with poor clinical outcome of laryngeal carcinoma and the co-prevalence of these viruses was significantly higher in lesions of oral squamous cell cancer and oral lichen planus compared to healthy mucosa (Szládek et al. 2005; Fehér et al. 2009). TTV DNA activates Toll-like receptor 9 (TLR9) and induces the production of different pro-inflammatory cytokines. Thus, TTV may affect the severity of diseases where inflammation plays an important role (Rocchi et al. 2009; Maggi and Bendinelli 2009). In addition, TTVs encode a microRNA (TTV-tth8 miRNA) that interferes with interferon signaling (Kincaid et al. 2013). TTV-tth8 miRNA may play a role in immune evasion by TTV and by TTV infected cells by interacting with the mRNA of a regulatory protein, N-myc (and STAT) interactor (NMI) that modulates interferon and cytokine signaling (Kincaid et al. 2013).

Hepatitis C virus (HCV), an enveloped, positive-sense single stranded RNA virus is a member of the Flaviviridae family; HCV causes chronic liver disease (Mohd Hanafiah et al. 2013). $\mathrm{HCV}$ infection is also associated with several extrahepatic manifestations (EHMs) (Zignego et al. 2007). One of the EHMs is oral lichen planus (OLP). It is worthy to note, however, that the association of HCV infection and OLP was stronger in Mediterranean countries and in Japan, whereas in northern Europe OLP was not associated with chronic liver disease caused by HCV (Carrozzo 2008). OLP is a chronic inflammatory disease that affects the skin and also the oral mucosa. The malignant transformation rate of 
Table 1 Viruses associated with head and neck carcinoma

\begin{tabular}{l|l|l}
\hline Virus & Major viral oncoproteins & Neoplasm or precancerous lesion \\
\hline $\begin{array}{l}\text { Human } \\
\text { papillomavirus }\end{array}$ & E6, E7 & $\begin{array}{l}\text { Oropharyngeal carcinoma, non-keratinizing; basaloid } \\
\text { phenotype }\end{array}$ \\
\hline Epstein-Barr virus & $\begin{array}{l}\text { LMP1, LMP2A, EBNA1 } \\
(?)\end{array}$ & Nasopharyngeal carcinoma, non-keratinizing type \\
\hline Torque teno virus & $?$ & Laryngeal carcinoma (?) \\
\hline Hepatitis C virus & $?$ & Oral lichen planus (?) \\
\hline
\end{tabular}

OLP was found to be quite low (1.09\%); still, it may play a role in the development of oral cancer (Fitzpatrick et al. 2014).

Further studies may clarify the role of TTV and HCV in HNSCC development.

Table 1 summarizes the viruses associated with head and neck carcinomas.

\section{Bacteria Associated with Head and Neck Carcinoma}

Although tobacco smoking, alcohol intake and HPV16 infection appear to be major, independent risk factors of a HNSCC, especially oral carcinoma, a series of observations indicate that oral bacteria and fungal infections of the oral cavity may also be associated, either casually or causally, with oral neoplasia. In a pioneering study, Nagy et al. analysed the biofilm flora present on the surfaces of oral squamous cell carcinomas and on the contiguous healthy mucosa (Nagy et al. 1998). They found a higher number of both aerobic and anaerobic colony forming units at the tumour sites than at the apparently healthy mucosa. They also compared the distribution of aerobic and anaerobic bacterial species at these anatomical sites. The frequency of most aerobic species was similar at both sites, except Serratia liquefaciens, Klebsiella pneumoniae, Citrobacter freundii, betahemolyzing Streptococci, and Enterococcus faecalis that were isolated more frequently from the biofilm samples obtained from the surfaces of oral carcinomas. Regarding anaerobic species, the frequency of peptostreptococci and lactobacilli was comparable at both sites, whereas Actinomyces spp., Propionibacterium spp., Clostridium spp., Veilonella spp., Fusobacterium spp., Prevotella spp., Porphyromonas spp., and Bacteriodes ureolyticus/gracilis was isolated more frequently from the tumor surface than from the control mucosal surface (Nagy et al. 1998). It is worthy to note that the fungus Candida albicans was detected in a significant fraction of oral carcinomas, but not at control sites (Nagy et al. 1998). Nagy et al. concluded that the cancer lesion itself may predispose patients with oral carcinoma to both local and systemic infections (Nagy et al. 1998). In a follow-up study, they demonstrated that topical antimicrobial treatment of oral squamous cell carcinoma lesions effectively reduced the number of biofilmassociated bacteria (Nagy et al. 2000). It is worthy to note that radiotherapy or cytostatic treatment of HNSCC patients also affected the composition of oral microbiota, resulting in an increased risk of local and systemic infections by pathogenic or opportunistic microbes (reviewed by Meurman 2010).

Hooper et al. aimed at the localization of bacteria within a surface-decontaminated oral squamous cell carcinoma (OSCC) sample; they performed in situ hybridization with a FITClabeled oligonucleotide recognizing a sequence within the 16S rRNA gene of Bacteria (Hooper et al. 2007). They found bacteria throughout the tumor tissue. Analysis of bacterial species by PCR cloning and sequencing of the $16 \mathrm{~S}$ rRNA gene revealed that there was a trend for an enrichment of Clavibacter michiganensis, Fusobacterium naviforme, Ralstonia insidiosa and Prevotella spp. in the tumor-derived samples whereas control tissue samples were enriched in Granulicatella adiacens, Porphyromonas 
gingivalis, Sphingomonas spp. and Streptococcus mitis/oralis (Hooper et al. 2007). Hooper et al. speculated the acidic and hypoxic microenvironment may select for the growth of certain bacterial species within tumors. They also raised the point that tumor-associated bacteria may play a role in carcinogenesis (Hooper et al. 2007). Others also emphasized that in addition to Helicobacter pylori, which is the causative agent of gastric carcinoma and gastric lymphoma in humans, other bacterial species could also be involved in tumorigenesis (Lax and Thomas 2002).

Pushalkar et al. used denaturing gradient gel electrophoresis and 16S rRNA gene sequencing to compare the oral microbiota of OSCC patients (Pushalkar et al. 2012). There were no significant differences in phylogenies at tumor and non-tumor sites, although four Streptococcus species as well as Peptostreptococcus stomatis, Gemella haemolysans, Gemella morbillorum, and Johnsonella ignova were highly associated with the tumor site. At the non-tumor site Granulicatella adiacens was prevalent. Pushalkar et al. noticed site- specific and subject-specific differences in the distribution of bacterial species. They suggested that certain oral bacteria may associate with different stages of OSCC and may contribute to the acidic and hypoxic milieu characteristic for neoplasms (Pushalkar et al. 2012).

Schmidt et al. performed pyrosequencing and also next generation sequencing (using the Illumina MiSeq instrument) to reveal the diversity of microbiomes in samples obtained by swabbing of oral cancer lesions and clinically normal mucosal surfaces (Schmidt et al. 2014). Based on the analysis of the V4 region of the bacterial $16 \mathrm{~S}$ rRNA genes, they classified 65,037 sequences at the genus level and 17,115 sequences at the species level. They observed a reduced abundance of the phyla Firmicutes (especially Streptococcus) and Actinobacteria (especially Rothia) in cancer and pre-cancer samples compared to the anatomically matched clinically normal patient samples. In contrast, the proportion of Fusobacteria increased at the tumor site. Although there were inter-individual differences, these changes appeared to be consistent. Schmidt et al. argued that in spite of the diversity of the oral microbial community, only distinct, biofilmforming oral bacteria adhere to oral tissues, followed by secondary colonizers. They suggested that altered surface properties at OSCC lesions may affect the adherence of bacteria, and a shift in bacterial populations may induce inflammatory responses favouring tumor progression (Schmidt et al. 2014). It is worthy to note that Fusobacterium nucleatum, a Gramnegative oral bacterium capable to invade the oral mucosa, was recently implicated in colon carcinogenesis (Castellarin et al. 2012; Kostic et al. 2012, 2013).

Bebek et al. amplified, cloned and sequenced variable regions $1-4$ of the prokaryotic $16 \mathrm{~S}$ rRNA gene to characterize bacterial populations in paired HNSCC and normal mucosa samples (Bebek et al. 2012). They also analysed the DNA methylation pattern of four cellular promoters (MDR1, IL8, RARB, TGFBR2) directing the expression of genes implicated in inflammation and tumorigenesis. Interestingly, hypermethylation of the MDRl promoter, a phenomenon regularly associated with promoter silencing, correlated with the presence of bacteria belonging to the Enterobacteriaceae family and the Tenericutes phylum (Bebek et al. 2012). MDRI codes for multidrug resistance protein 1, a drug efflux pump for xenobiotic compounds and MDR1 hypermethylation may contribute to the progression of gastric carcinoma (Tahara et al. 2009). Bebek et al. speculated that inflammatory processes elicited by bacteria may facilitate tumorigenesis by inducing hypermethylation of distinct cellular promoters (Bebek et al. 2012). It is worthy to note that the phylum Tenericutes includes the genera Mycoplasma and Ureaplasma which are prevalent in oral samples from STD patients (Nakashima et al. 2014). Others identified Mycoplasma salivarium as a dominant colonizer of oral carcinoma in two Fanconi anaemia patients, and it was also observed that Mycoplasma fermentans and Mycoplasma penetrans induced malignant cell transformation in vitro (Henrich et al. 2014; Tsai et al. 1995; Feng et al. 1999; Zhang et al. 1997). 
The distribution of bacterial species detected in the saliva may reflect the microbial diversity of the soft tissues located in the oral cavity. Thus, in principle, the salivary microbiota could be used as a diagnostic marker of OSCC. Mager et al. observed that the salivary counts of Capnocytophaga gingivalis, Prevotella melaninogenica, and Streptococcus mitis were elevated in the saliva of OSCC patients compared to the unstimulated saliva samples of OSCC-free subjects (Mager et al. 2005). Mager et al. speculated that alterations of tumor cell receptors may facilitate the adherence of certain bacteria and the resulting shift of soft tissue microbiota in the oral cavity may affect the levels of bacteria in the saliva (Mager et al. 2005). A recent study also found a shift in the saliva microbiome of OSCC patients: the most prevalent genera were Streptococcus, Gemella, Rothia, Peptostreptococcus, Lactobacillus, and Porphyromonas. In contrast, in control saliva samples Prevotella, Neisseria, Leptotrichia, Capnocytophaga, Actinobacillus, and Oribacterium dominated (Pushalkar et al. 2011).

Although the exact role of oral bacteria in carcinogenesis remains to be clarified, one potential mechanism is the generation of carcinogenic metabolites by certain oral bacteria (Meurman and Uittamo 2008). It was demonstrated that both non-pathogenic Neisseria strains and strains of Streptococcus salivarius and Streptococcus intermedius as well as Corynebacterium spp. and Stomatococcus spp. are capable to convert ethanol to acetaldehyde, a mutagenic and carcinogenic substance (Muto et al. 2000; Homann et al. 2000; Kurkivuori et al. 2007). Such a mechanism may explain how poor dental status associated with bacterial overgrowth may increase oral cancer risk in patients with tooth loss, poor dentition and inadequate oral hygiene (Homann et al. 2000, 2001). Homann et al. demonstrated an increase in salivary acetaldehyde production from ethanol in saliva samples of patients with poor dental status (Homann et al. 2001).

Table 2 summarizes the bacteria associated with head and neck carcinomas and their putative role in carcinogenesis.

\section{Fungi Associated with Head and Neck Carcinoma}

In parallel to the alterations of the bacterial flora in oral cancer (see above), Nagy et al. described the presence of Candida albicans in a significant fraction of oral carcinomas, but not at control sites (Nagy et al. 1998). Furthermore, similarly to certain oral bacteria, it was documented that both Candida albicans strains and non-Candida albicans yeasts were capable of salivary acetaldehyde production from ethanol, and Candida albicans could frequently be detected in oral epithelial dysplasia, a premalignant lesion with an increased risk of oral cancer (Tillonen et al. 1999; McCullough et al. 2002; Nieminen et al. 2009, reviewed by Sitheeque and Samaranayake 2003; Bakri et al. 2010).

Chronic hyperplastic candidiasis $(\mathrm{CHC}$, also referred to as candidal leukoplakia) is a clinical term for Candida-infected oral leukoplakias of the oral mucosa that are characterized by hyphal invasion and parakeratinosis. Although most frequently Candida albicans could be detected in CHC lesions, other species including Candida dubliniensis, Candida tropicalis, Candida pintolopesii, Candida glabrata and Sacharomyces cerevisiae were also detected in adherent chronic white patches of the oral mucosa (Cernea et al. 1965; Jepsen and Winther 1965; Krogh et al. 1986, reviewed by Bakri et al. 2010). C. albicans may either colonize existing premalignant or malignant oral lesions, or may promote the generation of precancerous conditions and their progression to cancer (Cernea et al. 1965; Jepsen and Winther 1965; Nagy et al. 1998; Sitheeque and Samaranayake 2003; Bakri et al. 2010; Sanjaya et al. 2011). A strong argument for a carcinogenic role of Candida infection is the production of carcinogens by certain Candida spp.: in addition to the mutagenic acetaldehyde (see above), formation of the potent carcinogen $\mathrm{N}$-nitrosobenzylmethylamine (NBMA) was also observed (Krogh et al. 1987; Krogh 1990). Production of proteinases and pro-inflammatory mediators by Candida spp. may also contribute, indirectly, to carcinogenesis 
Table 2 Bacteria associated with oral carcinomas and their putative role in carcinogenesis or tumor progression

\begin{tabular}{|c|c|c|}
\hline Bacterium & Putative role & Reference \\
\hline Serratia liquefaciens & $?$ & \multirow[t]{13}{*}{ Nagy et al. (1998) } \\
\hline Klebsiella pneumoniae & $?$ & \\
\hline Citrobacter freundii & $?$ & \\
\hline $\begin{array}{l}\text { beta-hemolyzing } \\
\text { Streptococci }\end{array}$ & $?$ & \\
\hline Enterococcus faecalis & $?$ & \\
\hline Actinomyces spp. & $?$ & \\
\hline Propionibacterium spp. & $?$ & \\
\hline Clostridium spp. & $?$ & \\
\hline Veilonella spp. & $?$ & \\
\hline Fusobacterium spp. & $?$ & \\
\hline Prevotella spp. & $?$ & \\
\hline Porphyromonas spp. & $?$ & \\
\hline $\begin{array}{l}\text { Bacteriodes ureolyticus/ } \\
\text { gracilis }\end{array}$ & $?$ & \\
\hline $\begin{array}{l}\text { Clavibacter } \\
\text { michiganensis }\end{array}$ & $?$ & \multirow[t]{4}{*}{ Hooper et al. (2007) } \\
\hline $\begin{array}{l}\text { Fusobacterium } \\
\text { naviforme }\end{array}$ & $?$ & \\
\hline Ralstonia insidiosa & $?$ & \\
\hline Prevotella spp. & $?$ & \\
\hline Streptococcus spp. & \multirow[t]{4}{*}{ Contribution to the acidic and hypoxic milieu } & \multirow[t]{4}{*}{ Pushalkar et al. (2012) } \\
\hline $\begin{array}{l}\text { Peptostreptococcus } \\
\text { stomatis }\end{array}$ & & \\
\hline $\begin{array}{l}\text { Gemella haemolysans, } \\
\text { Gemella morbillorum }\end{array}$ & & \\
\hline Johnsonella ignova & & \\
\hline Fusobacterium spp. & $?$ & Schmidt et al. (2014) \\
\hline $\begin{array}{l}\text { Enterobacteriaceae } \\
\text { (family) }\end{array}$ & \multirow[t]{2}{*}{$\begin{array}{l}\text { Induction of cellular promoter hypermethylation; } \\
\text { induction of pro-inflammatory changes }\end{array}$} & \multirow[t]{2}{*}{ Bebek et al. (2012) } \\
\hline Tenericutes (phylum) & & \\
\hline Streptococcus salivarius & \multirow[t]{4}{*}{ Conversion of ethanol to mutagenic acetaldehyde } & \multirow{4}{*}{$\begin{array}{l}\text { Muto et al. (2000), Homann } \\
\text { et al. (2000), Kurkivuori } \\
\text { et al. (2007) }\end{array}$} \\
\hline $\begin{array}{l}\text { Streptococcus } \\
\text { intermedius }\end{array}$ & & \\
\hline Corynebacterium spp. & & \\
\hline Stomatococcus spp. & & \\
\hline
\end{tabular}

Table 3 Fungi associated with oral carcinomas and their putative role in carcinogenesis

\begin{tabular}{l|l|l}
\hline Fungus & Putative role & References \\
\hline \multirow{2}{*}{ Candida spp. } & Salivary acetaldehyde production from ethanol & Tillonen et al. (1999) \\
\cline { 2 - 3 } & Formation of carcinogen (nitrosobenzylmethylamine, NBMA) & Krogh et al. (1987), Krogh (1990) \\
\hline
\end{tabular}

by degrading cell surface proteins, basement membrane and extracellular matrix components and by eliciting chronic inflammation (reviewed by Bakri et al. 2010). Table 3 summarizes the fungi associated with head and neck carcinomas and their putative role in carcinogenesis

\section{Conclusions}

In the head and neck region, besides traditional risk factors such as smoking habits and alcohol consumption, certain microbes also play a role in 
the generation of malignant epithelial tumors. Infection with high-risk human papillomavirus types is strongly associated with the development of oropharyngeal carcinoma and Epstein-Barr virus appears to be indispensable for the development of non-keratinizing squamous cell carcinoma of the nasopharynx. Infection with other viruses including torque teno virus and hepatitis $\mathrm{C}$ virus may increase the risk of initiation or progression head and neck carcinomas. A shift in the composition of the oral microbiome was also associated with oral squamous cell carcinoma, although the exact role of oral bacteria remains to be clarified. Conversion of ethanol to acetaldehyde, a mutagenic compound, by members of the oral microflora as well as by fungi including Candida albicans and others may increase oral cancer risk. In addition, distinct Candida spp. also produce NBMA (N-nitrosobenzylmethylamine), a potent carcinogen. Inflammatory processes elicited by microbes may also facilitate tumorigenesis in the head and neck region.

\section{References}

Alibek K, Kakpenova A, Baiken Y (2013) Role of infectious agents in the carcinogenesis of brain and head and neck cancers. Infect Agent Cancer 8:7-15

Ang KK, Harris J, Wheeler R, Weber R, Rosenthal DI, Nguyen-Tân PF, Westra WH, Chung CH, Jordan RC, Lu C, Kim H, Axelrod R, Silverman CC, Redmond KP, Gillison ML (2010) Human papillomavirus and survival of patients with oropharyngeal cancer. N Engl J Med 363:24-35

Bakri MM, Hussaini HM, Holmes AR, Cannon RD, Rich AM (2010) Revisiting the association between candidal infection and carcinoma, particularly oral squamous cell carcinoma. J Oral Microbiol 2:5780

Bebek G, Bennett K, Funchain P, Campbell R, Seth R, Scharpf J, Burkey B, Eng C (2012) Microbiomic subprofiles and MDR1 promoter methylation in head and neck squamous cell carcinoma. Hum Mol Genet 20:1557-1565

Bose P, Brockton NT, Dort JC (2013) Head and neck cancer: from anatomy to biology. Int $\mathrm{J}$ Cancer 133:2013-2023

Boyer SN, Wazer DE, Band V (1996) E7 protein of human papilloma virus-16 induces degradation of retinoblastoma protein through the ubiquitin-proteasome pathway. Cancer Res 56:4620-4624
Braakhuis BJ, Tabor MP, Kummer JA, Leemans CR, Barkenhoff RH (2003) A genetic explanation of Slaughter's concept of field cancerization: evidence and clinical implications. Cancer Res 63:1727-1730

Burke SC, Vail Smith K, Sharmin S, Winkelman C (2014) Prevalence of risk factors related to Head and Neck Squamous Cell Carcinoma (HNSCC) among college students. Cancer and Oncology Research 2:7-16

Bzhalava D, Guan P, Franceschi S, Dillner J, CliffordG (2013) A systematic review of the prevalence of mucosal and cutaneous human papillomavirus types. Virology 445:224-231

Cadoni G, Boccia S, Petrelli L, Di Giannantonio P, Arzani D, Giorgio A, De Feo E, Pandolfini M, Gallì P, Paludetti G, Ricciardi G (2012) A review of genetic epidemiology of head and neck cancer related to polymorphisms in metabolic genes, cell cycle control and alcohol metabolism. Acta Otorhinolaryngol Ital 32:1-11

Caicedo-Granados E, Lin R, Fujisawa C, Yueh B, Sangwan V, Saluja A (2014) Wild-type p53 reactivation by small-molecule Minnelide ${ }^{\mathrm{TM}}$ in human papillomavirus (HPV)-positive head and neck squamous cell carcinoma. Oral Oncol 50:1149-1156

Campo MS, Graham SV, Cortese MS, Ashrafi GH, Araibi EH, Dornan ES, Miners K, Nunes C, Man S (2010) HPV-16 E5 down-regulates expression of surface HLA class I and reduces recognition by CD8 T cells. Virology 407:137-142

Carrozzo M (2008) Oral diseases associated with hepatitis $\mathrm{C}$ virus infection. Part 2: lichen planus and other diseases. Oral Dis 14:217-228

Castellarin M, Warren RL, Freeman JD, Dreolini L, Krzywinski M, Strauss J, Barnes R, Watson P, Allen-Vercoe E, Moore RA, Holt RA (2012) Fusobacterium nucleatum infection is prevalent in human colorectal carcinoma. Genome Res 22:299-306

Cernea P, Crepy C, Kuffer R, Mascaro JM, Badillet G, Marie JL (1965) Little known aspects of oral candidiasis. The candidiasis with multiple foci of the oral cavity. Rev Stomatol Chir Maxillofac 66:103-108. (Article in French)

Chaturvedi AK (2012) Epidemiology and clinical aspects of HPV in head and neck cancers. Head Neck Pathol 6 (Suppl 1):S16-S24

Dalal KM, Antonescu CR, DeMatteo RP, Maki RG (2008) EBV-associated smooth muscle neoplasms: solid tumors arising in the presence of immunosuppression and autoimmune diseases. Sarcoma 2008. Article ID 859407

de Martel C, Ferlay J, Franceschi S, Vignat J, Bray F, Forman D, Plummer M (2012) Global burden of cancers attributable to infections in 2008: a review and synthetic analysis. Lancet Oncol 13:607-615

de Villiers EM, Schmidt R, Delius H, zur Hausen H (2002) Heterogeneity of TT virus related sequences isolated from human tumour biopsy specimens. J Mol Med (Berl) 80:44-50 
de Villiers EM, Bulajic M, Nitsch C, Kecmanovic D, Pavlov M, Kopp-Schneider A, Löhr M (2007) TTV infection in colorectal cancer tissues and normal mucosa. Int J Cancer 121:2109-2112

Deng Z, Hasegawa M, Kiyuna A, Matayoshi S, Uehara T, Agena S, Yamashita Y, Ogawa K, Maeda H, Suzuki M (2013) Viral load, physical status, and E6/E7 mRNA expression of human papillomavirus in head and neck squamous cell carcinoma. Head Neck 35:800-808

DiMaio D, Mattoon D (2001) Mechanisms of cell transformation by papillomavirus E5 proteins. Oncogene 20:7866-7873

El-Mofty SK (2012) HPV-related squamous cell carcinoma variants in the head and neck. Head Neck Pathol 6:S55-S62

Elrefaey S, Massaro MA, Chiocca S, Chiesa F, Ansarin M (2014) HPV in oropharyngeal cancer: the basics to know in clinical practice. Acta Otorhinolaryngol Ital 34:299-309

Epstein MA, Achong BG, Barr YM (1964) Virus particles in cultured fibroblasts from Burkitt's lymphoma. Lancet 1:702-703

Fakhry C, Psyrri A, Chaturvedhi A (2014) HPV and head and neck cancers: state-of-the-science. Oral Oncol 50:353-355

Fehér E, Gáll T, Murvai M, Kis A, Boda R, Sápy T, Tar I, Gergely L, Szarka K (2009) Investigation of the occurrence of torque teno virus in malignant and potentially malignant disorders associated with human papillomavirus. J Med Virol 81:1975-1981

Feng SH, Tsai S, Rodriguez J, Lo SC (1999) Mycoplasma infections prevent apoptosis and induce malignant transformation of interleukin-3-dependent 32D hematopoietic cells. Mol Cell Biol 19:7995-8002

Ferlay J, Shin HR, Bray F, Forman D, Mathers C, Parkin DM (2010) Estimates of worldwide burden of cancer in 2008: GLOBOCAN 2008. Int $\mathrm{J}$ Cancer 127:2893-2917

Figueiredo CP, Franz-Vasconcelos HC, Giunta G, Mazzuco TL, Caon T, Fernandes AL, Simões CM, Antunes VL, Niel C, Barardi CR (2007) Detection of Torque teno virus in Epstein-Barr virus positive and negative lymph nodes of patients with Hodgkin lymphoma. Leuk Lymphoma 48:731-735

Fitzpatrick SG, Hirsch SA, Gordon SC (2014) The malignant transformation of oral lichen planus and oral lichenoid lesions: a systematic review. J Am Dent Assoc 145:45-56

Genden EM, Sambur IM, de Almeida JR, Posner M, Rinaldo A, Rodrigo JP, Strojan P, Takes RP, Ferlito A (2013) Human papillomavirus and oropharyngeal squamous cell carcinoma: what the clinician should know. Eur Arch Otorhinolaryngol 270:405-416

Gergely P Jr, Perl A, Poór G (2006) Possible pathogenic nature of the recently discovered TT virus: does it play a role in autoimmune rheumatic diseases? Autoimmun Rev 6:5-9

Gillison ML, D’Souza G, Westra W, Sugar E, Xiao W, Begum S, Viscidi R (2008) Distinct risk factor profiles for human papillomavirus type 16-positive and human papillomavirus type 16-negative head and neck cancers. J Natl Cancer Inst 100:407-420

Gillison ML, Broutian T, Pickard RK, Tong ZY, Xiao W, Kahle L, Graubard BI, Chaturvedi AK (2012) Prevalence of oral HPV infection in the United States, 2009-2010. JAMA 307:693-703

Gillison ML, Castellsagué X, Chaturvedi A, Goodman MT, Snijders P, Tommasino M, Arbyn M, Franceschi S (2014) Eurogin Roadmap: comparative epidemiology of HPV infection and associated cancers of the head and neck and cervix. Int J Cancer 134:497-507

Hanahan D, Weinberg RA (2000) The hallmarks of cancer. Cell 100:57-70

Henrich B, Rumming M, Sczyrba A, Velleuer E, Dietrich R, Gerlach W, Gombert M, Rahn S, Stove J, Borkhardt A, Fischer U (2014) Mycoplasma salivarium as a dominant colonizer of Fanconi anemia associated oral carcinoma. PLoS One 9(3):e92297

Ho JH, Huang DP, Fong YY (1978) Sated fish and nasopharyngeal carcinoma in southern Chinese. Lancet 2:626

Homann N, Tillonen J, Meurman JH, Rintamäki H, Lindquist C, Rautio M, Jousimies-Somer H, Salaspuro M (2000) Increased salivary acetaldehyde levels in heavy drinkers and smokers: a microbiological approach to oral cavity cancer. Carcinogenesis 21:663-668

Homann N, Tillonen J, Rintamäki H, Salaspuro M, Lindquist C, Meurman JH (2001) Poor dental status increases acetaldehyde production from ethanol in saliva: a possible link to increased oral cancer risk among heavy drinkers. Oral Oncol 37:153-158

Hooper SJ, Crean SJ, Fardy MJ, Lewis MAO, Spratt DA, Wade WG, Wilson MJ (2007) A molecular analysis of the bacteria present within oral squamous cell carcinoma. J Med Microbiol 56:1651-1659

Hsieh SY, Wu YH, Ho YP, Tsao KC, Yeh CT, Liaw YF (1999) High prevalence of TT virus infection in healthy children and adults and in patients with liver disease in Taiwan. J Clin Microbiol 37:1829-1831

Hsu CY, Yi YH, Chang KP, Chang YS, Chen SJ, Chen HC (2014) The Epstein-Barr virus-encoded microRNA miR-BART9 promotes tumor metastasis by targeting E-cadherin in nasopharyngeal carcinoma. PLoS Pathog 10(2):e1003974

Hussain T, Manzoor S, Waheed Y, Tariq H, Hanif K (2012) Phylogenetic analysis of Torque Teno virus genome from Pakistani isolate and incidence of co-infection among $\mathrm{HBV} / \mathrm{HCV}$ infected patients. Virol J 9:320-329

IARC Working Group on the Evaluation of Carcinogenic Risks to Humans (2012) Biological agents. Volume 100 B. A review of human carcinogens. IARC Monogr Eval Carcinog Risks Hum 100(Pt B):1-441

Iriti M, Varoni EM (2015) Is there any relationship between the type of alcoholic beverage and oral cancer? Focus on red wine in an European perspective. Pol J Food Nutr Sci 65:5-7 
Isayeva T, Li Y, Maswahu D, Brandwein-Gensler M (2012) Human papillomavirus in non-oropharyngeal head and neck cancers: a systematic literature review. Head Neck Pathol 6, Suppl 1:S104-S120

Ito Y, Ohigashi H, Koshimizu K, Yi Z (1983) Epstin-Barr virus-activating principle in the ether extracts of soils collected from under plants which contain active diterpene esters. Cancer Lett 19:113-117

Jalouli J, Ibrahim SO, Mehrotra R, Jalouli MM, Sapkota D, Larsson PA, Hirsch JM (2010) Prevalence of viral (HPV, EBV, HSV) infections in oral submucous fibrosis and oral cancer from India. Acta Otolaryngol 130:1306-1311

Jepsen A, Winther JE (1965) Mycotic infection in oral leukoplakia. Acta Odontol Scand 23:239-256

Jia WH, Qin HD (2012) Non-viral environmental risk factors for nasopharyngeal carcinoma: a systematic review. Semin Cancer Biol 22:117-126

Joshi P, Dutta S, Chaturvedi P, Nair S (2014) Head and neck cancers in developing countries. Rambam Maimonides Med J 5(2):1-6

Kincaid RP, Burke JM, Cox JC, de Villiers EM, Sullivan CS (2013) A human torque teno virus encodes a microRNA that inhibits interferon signaling. PLoS Pathog 9:e1003818

Klingelhutz AJ, Roman A (2012) Cellular transformation by human papillomaviruses: lessons learned by comparing high- and low-risk viruses. Virology 424:77-98

Klussmann JP, Weissenborn SJ, Wieland U, Dries V, Kolligs J, Jungehuelsing M, Eckel HE, Dienes HP, Pfister HJ, Fuchs PG (2001) Prevalence, distribution, and viral load of human papillomavirus 16 DNA in tonsillar carcinomas. Cancer 92:2875-2884

Kostic AD, Gevers D, Pedamallu CS, Michaud M, Duke F, Earl AM, Ojesina AI, Jung J, Bass AJ, Tabernero J, Baselga J, Liu C, Shivdasani RA, Ogino S, Birren BW, Huttenhower C, Garrett WS, Meyerson M (2012) Genomic analysis identifies association of Fusobacterium with colorectal carcinoma. Genome Res 22:292-298

Kostic AD, Chun E, Robertson L, Glickman JN, Gallini CA, Michaud M, Clancy TE, Chung DC, Lochhead P, Hold GL, El-Omar EM, Brenner D, Fuchs CS, Meyerson M, Garrett WS (2013) Fusobacterium nucleatum potentiates intestinal tumorigenesis and modulates the tumor-immune microenvironment. Cell Host Microbe 14:207-215

Kreimer AR, Clifford GM, Boyle P, Franceschi S (2005) Human papillomavirus types in head and neck squamous cell carcinomas worldwide: a systematic review. Cancer Epidemiol Biomarkers Prev 14:467-475

Krogh P (1990) The role of yeasts in oral cancer by means of endogenous nitrosation. Acta Odontol Scand 48:85-88

Krogh P, Hald B, Holmstrup P (1987) Possible mycological etiology of oral mucosal cancer: catalytic potential of infecting Candida albicans and other yeasts in production of N-nitrosobenzylmethylamine. Carcinogenesis 8:1543-1548
Krogh P, Holmstrup P, Vedtofte P, Pindborg JJ (1986) Yeast organisms associated with human oral leukoplakia. Acta Derm Venereol Suppl (Stockh) 121:51-55

Kurkivuori J, Salaspuro V, Kaihovaara P, Kari K, Rautemaa R, Grönroos L, Meurman J, Salaspuro M (2007) Acetaldehyde production from ethanol by oral streptococci. Oral Oncol 43:181-186

Lax AJ, Thomas W (2002) How bacteria could cause cancer: one step at a time. Trends Microbiol 10:294-299

Lazarczyk M, Cassonnet P, Pons C, Jacob Y, Favre M (2009) The EVER proteins as a natural barrier against papillomaviruses: a new insight into the pathogenesis of human papillomavirus infections. Microbiol Mol Biol Rev 73:348-370

Leemans CR, Braakhuis BJ, Brakenhoff RH (2011) The molecular biology of head and neck cancer. Nat Rev Cancer 11:9-22

Li C, Johnson DE (2013) Liberation of functional p53 by proteasome inhibition in human papilloma viruspositive head and neck squamous cell carcinoma cells promotes apoptosis and cell cycle arrest. Cell Cycle 12:923-934

Li LL, Shu XS, Wang ZH, Cao Y, Tao Q (2011) Epigenetic disruption of cell signaling in nasopharyngeal carcinoma. Chin J Cancer 30:231-239

Li WC, Lee PL, Chou IC, Chang WJ, Lin SC, Chang KW (2015) Molecular and cellular cues of diet-associated oral carcinogenesis-with an emphasis on areca-nutinduced oral cancer development. J Oral Pathol Med 44:167-177

Lindquist D, Romanitan M, Hammarstedt L, Näsman A, Dahlstrand H, Lindholm J, Onelöv L, Ramqvist T, Ye W, Munck-Wikland E, Dalianis T (2007) Human papillomavirus is a favourable prognostic factor in tonsillar cancer and its oncogenic role is supported by the expression of E6 and E7. Mol Oncol 1:350-355

Lo KW, Chung GT, To KF (2012) Deciphering the molecular genetic basis of NPC through molecular, cytogenetic, and epigenetic approaches. Semin Cancer Biol 22:79-86

López RV, Levi JE, Eluf-Neto J, Koifman RJ, Koifman S, Curado MP, Michaluart-Junior P, Figueiredo DL, Saggioro FP, de Carvalho MB, Kowalski LP, Abrahão M, de Góis-Filho F, Tajara EH, Waterboer T, Boffetta P, Brennan P, Wünsch-Filho V (2014) Human papillomavirus (HPV) 16 and the prognosis of head and neck cancer in a geographical region with a low prevalence of HPV infection. Cancer Causes Control 25:461-471

Louie KS, Mehanna H, Sasieni P (2015) Trends in head and neck cancers in England from 1995 to 2011 and projections up to 2025. Oral Oncol 51:341-348

Lung ML, Cheung AK, Ko JM, Lung HL, Cheng Y, Dai W (2014) The interplay of host genetic factors and Epstein-Barr virus in the development of nasopharyngeal carcinoma. Chin J Cancer 33:556-568

Mager DI, Haffajee AD, Devlin PM, Norris CM, Posner MR, Goodson JM (2005) The salivary microbiota as 
an indicator of oral cancer: a descriptive, non-randomized study of cancer free and oral squamous cell carcinoma subjects. J Transl Med 3:27

Maggi F, Bendinelli M (2009) Immunobiology of the Torque teno viruses and other anelloviruses. Curr Top Microbiol Immunol 331:65-90

Majchrzak E, Szybiak B, Wegner A, Pienkowski P, Pazdrowski J, Luczewski L, Sowka M, Golusinski P, Malicki J, Golusinski W (2014) Oral cavity and oropharyngeal squamous cell carcinoma in young adults: a review of the literature. Radiol Oncol 48:1-10

Marur S, D'Souza G, Westra WH, Forastiere AA (2010) HPV-associated head and neck cancer: a virus-related cancer epidemic. Lancet Oncol 11:781-789

Maurya SS, Anand G, Dhawan A, Khan AJ, Jain SK, Pant MC, Parmar D (2014) Polymorphisms in drugmetabolizing enzymes and risk to head and neck cancer: evidence for gene-gene and gene-environment interaction. Environ Mol Mutagen 55:134-144

McCullough M, Jaber M, Barrett AW, Bain L, Speight PM, Porter SR (2002) Oral yeast carriage correlates with presence of oral epithelial dysplasia. Oral Oncol 38:391-393

McLaughlin-Drubin ME, Münger K (2010) Oncogenic activities of human papillomaviruses. Virus Res 143:195-208

Mehanna H, Beech T, Nicholson T, El-Hariry I, McConkey C, Paleri V, Roberts S (2013) Prevalence of human papillomavirus in oropharyngeal and nonoropharyngeal head and neck cancer--systematic review and meta-analysis of trends by time and region. Head Neck 35:747-755

Mellin H, Dahlgren L, Munck-Wikland E, Lindholm J, Rabbani H, Kalantari M, Dalianis T (2002) Human papillomavirus type 16 is episomal and a high viral load may be correlated to better prognosis in tonsillar cancer. Int J Cancer 102:152-158

Meurman JH (2010) Infectious and dietary risk factors of oral cancer. Oral Oncol 46:411-413

Meurman JH, Uittamo J (2008) Oral micro-organisms in the etiology of cancer. Acta Odontol Scand 66:321-326

Moen EM, Sleboda J, Grinde B (2002) Serum concentrations of TT virus and TT virus-like mini virus in patients developing AIDS. AIDS 16:1679-1682

Mohd Hanafiah K, Groeger J, Flaxman AD, Wiersma ST (2013) Global epidemiology of hepatitis C virus infection: new estimates of age-specific antibody to HCV seroprevalence. Hepatology 57:1333-1342

Moody CA, Laimins LA (2010) Human papillomavirus oncoproteins: pathways to transformation. Nat Rev Cancer 10:550-560

Muto M, Hitomi Y, Ohtsu A, Shimada H, Kashiwase Y, Sasaki H, Yoshoda S, Esumi H (2000) Acetaldehyde production by non-pathogenic Neisseria in human oral microflora: implications for carcinogenesis in upper aerodigestive tract. Int J Cancer 88:342-350
Nagy KN, Sonkodi I, Szöke I, Nagy E, Newman HN (1998) The microflora associated with human oral carcinomas. Oral Oncol 34:304-308

Nagy K, Szöke I, Sonkodi I, Nagy E, Mari A, Szolnoky G, Newman HN (2000) Inhibition of microflora associated with oral malignancy. Oral Oncol 36:32-36

Nakashima K, Shigehara K, Kawaguchi S, Wakatsuki A, Kobori Y, Nakashima K, Ishii Y, Shimamura M, Sasagawa T, Kitagawa Y, Mizokami A, Namiki M (2014) Prevalence of human papillomavirus infection in the oropharynx and urine among sexually active men: a comparative study of infection by papillomavirus and other organisms, including Neisseria gonorrhoeae, Chlamydia trachomatis, Mycoplasma spp. and Ureaplasma spp. BMC Infect Dis 14:43

Näsman A, Attner P, Hammarstedt L, Du J, Eriksson M, Giraud G, Ahrlund-Richter S, Marklund L, Romanitan M, Lindquist D, Ramqvist T, Lindholm J, Sparén P, Ye W, Dahlstrand H, Munck-Wikland E, Dalianis T (2009) Incidence of human papillomavirus (HPV) positive tonsillar carcinoma in Stockholm, Sweden: an epidemic of viral-induced carcinoma? Int J Cancer 125:362-366

Nicholls JM, Agathanggelou A, Fung K, Zeng X, Niedobitek G (1997) The association of squamous cell carcinomas of the nasopharynx with EpsteinBarr virus shows geographical variation reminiscent of Burkitt's lymphoma. J Pathol 183:164-168

Nieminen MT, Uittamo J, Salaspuro M, Rautemaa R (2009) Acetaldehyde production from ethanol and glucose by non-Candida albicans yeasts in vitro. Oral Oncol 45:e245-e249

Niller HH, Wolf H, Minarovits J (2007) Epstein-Barr virus. In: Minarovits J, Gonczol E, Valyi-Nagy T (eds) Latency strategies of herpesviruses. Springer, New York, pp 154-191

Niller HH, Wolf H, Minarovits J (2009) Epigenetic dysregulation of the host cell genome in EpsteinBarr virus associated neoplasia. Semin Cancer Biol 19:158-164

Niller HH, Banati F, Minarovits J (2014) Epigenetic alterations in nasopharyngeal carcinoma and EpsteinBarr virus (EBV) associated gastric carcinoma: a lesson in contrasts. J Nasophyryng Carcinoma 1(9):e9

Ninomiya M, Takahashi $M$, Nishizawa $T$, Shimosegawa T, Okamoto H (2008) Development of PCR assays with nested primers specific for differential detection of three human anelloviruses and early acquisition of dual or triple infection during infancy. $\mathrm{J}$ Clin Microbiol 246:507-514

Nishizawa T, Okamoto H, Konishi K, Yoshizawa H, Miyakawa Y, Mayumi M (1997) A novel DNA virus (TTV) associated with elevated transaminase levels in posttransfusion hepatitis of unknown etiology. Biochem Biophys Res Commun 241:92-97

Okamoto H (2009) History of discoveries and pathogenicity of TT viruses. Curr Top Microbiol Immunol 331:1-20 
Olthof NC, Huebbers CU, Kolligs J, Henfling M, Ramaekers FC, Cornet I, van Lent-Albrechts JA, Stegmann AP, Silling S, Wieland U, Carey TE, Walline HM, Gollin SM, Hoffmann TK, de Winter J, Kremer B, Klussmann JP, Speel EJ (2015) Viral load, gene expression and mapping of viral integration sites in HPV16-associated HNSCC cell lines. Int J Cancer 136:E207-E218

Paluszczak J, Misiak P, Wierbicka M, Wozniak A, BaerDubrowska W (2011) Frequent hypermethylation of DAPK, RARbeta, MGMT, RASSF1A and FHIT in laryngeal squamous cell carcinomas and adjacent normal mucosa. Oral Oncol 47:104-107

Parfenov M, Pedamallu CS, Gehlenborg N, Freeman SS, Danilova L, Bristow CA, Lee S, Hadjipanayis AG, Ivanova EV, Wilkerson MD, Protopopov A, Yang L, Seth S, Song X, Tang J, Ren X, Zhang J, Pantazi A, Santoso N, Xu AW, Mahadeshwar H, Wheeler DA, Haddad RI, Jung J, Ojesina AI, Issaeva N, Yarbrough WG, Hayes DN, Grandis JR, El-Naggar AK, Meyerson M, Park PJ, Chin L, Seidman JG, Hammerman PS, Kucherlapati R, Cancer Genome Atlas Network (2014) Characterization of HPV and host genome interactions in primary head and neck cancers. Proc Natl Acad Sci U S A 111:15544-15549

Pifferi M, Maggi F, Andreoli E, Lanini L, Marco ED, Fornai C, Vatteroni ML, Pistello M, Ragazzo V, Macchia P, Boner A, Bendinelli M (2005) Associations between nasal torquetenovirus load and spirometric indices in children with asthma. J Infect Dis 192:1141-1148

Pushalkar S, Mane SP, Ji X, Li Y, Evans C, Crasta OR, Morse D, Meagher RM, Singh A, Saxena D (2011) Microbial diversity in saliva of oral squamous cell carcinoma. FEMS Immunol Med Microbiol 61:269-277

Pushalkar S, Ji X, Li Y, Estilo C, Yegnanarayana R, Singh B, Li X, Saxena D (2012) Comparison of oral microbiota in tumor and non-tumor tissues of patients with squamous cell carcinoma. BMC Microbiol $12: 144$

Rampias T, Sasaki C, Weinberger P, Psyrri A (2009) E6 and E7 gene silencing and transformed phenotype of human papillomavirus 16-positive oropharyngeal cancer cells. J Natl Cancer Inst 101:412-423

Ramqvist T, Grün N, Dalianis T (2015) Human papillomavirus and tonsillar and base of tongue cancer. Viruses 7:1332-1343

Rautava J, Syrjänen S (2012) Biology of human papillomavirus infections in head and neck carcinogenesis. Head Neck Pathol 6(Suppl 1):3-15

Ribeiro KB, Levi JE, Pawlita M, Koifman S, Matos E, Eluf-Neto J, Wunsch-Filho V, Curado MP, Shangina O, Zaridze D, Szeszenia-Dabrowska N, Lissowska J, Daudt A, Menezes A, Bencko V, Mates D, Fernandez L, Fabianova E, Gheit T, Tommasino M, Boffetta P, Brennan P, Waterboer T (2011) Low human papillomavirus prevalence in head and neck cancer: results from two large case-control studies in high-incidence regions. Int $\mathrm{J}$ Epidemiol 40:489-502

Rietbergen MM, Leemans CR, Bloemena E, Heideman DA, Braakhuis BJ, Hesselink AT, Witte BI, Baatenburg de Jong RJ, Meijer CJ, Snijders PJ, Brakenhoff RH (2013) Increasing prevalence rates of HPV attributable oropharyngeal squamous cell carcinomas in the Netherlands as assessed by a validated test algorithm. Int J Cancer 132:1565-1571

Rocchi J, Ricci V, Albani M, Lanini L, Andreoli E, Macera L, Pistello M, Ceccherini-Nelli L, Bendinelli M, Maggi F (2009) Torque teno virus DNA drives proinflammatory cytokines production and secretion by immune cells via toll-like receptor 9. Virology 394:235-242

Saback FL, Gomes SA, de Paula VS, da Silva RR, LewisXimenez LL, Niel C (1999) Age-specific prevalence and transmission of TT virus. J Med Virol 59:316-322

Safaeian M, Porras C, Schiffman M, Rodriguez AC, Wacholder S, Gonzalez P, Quint W, van Doorn LJ, Sherman ME, Xhenseval V, Herrero R, Hildesheim A, Costa Rican Vaccine Trial Group (2010) Epidemiological study of anti-HPV16/18 seropositivity and subsequent risk of HPV16 and -18 infections. J Natl Cancer Inst 102:1653-1662

Saláková M, Nemecek V, Tachezy R (2009) TTV and HPV co-infection in cervical smears of patients with cervical lesions. BMC Infect Dis 9:118-123

Sand L, Jalouli J (2014) Viruses and oral cancer. Is there a link? Microbes Infect 16:371-378

Sand L, Wallström M, Hirsch JM (2014) Smokeless tobacco, viruses and oral cancer. Oral Health Dent Manag 13:372-378

Sanjaya PR, Gokul S, Patil G, Raju R (2011) Candida in oral pre-cancer and cancer. Med Hypotheses 77:1125-1128

Schmidt BL, Kuczynski J, Bhattacharya A, Huey B, Corby PM, Queiroz ELS, Nightingale K, Kerr AR, DeLacure MD, Veermachaneni R, Olshen A, Albertson DG (2014) Changes in abundance of oral microbiota associated with oral cancer. PLoS One 9 (6):e98741

Sethi S, Ali-Fehmi R, Franceschi S, Struijk L, van Doorn LJ, Quint W, Albashiti B, Ibrahim M, Kato I (2012) Characteristics and survival of head and neck cancer by HPV status: a cancer registry-based study. Int $\mathbf{J}$ Cancer 131:1179-1186

Shah KM, Young LS (2009) Epstein-Barr virus and carcinogenesis: beyond Burkitt's lymphoma. Clin Microbiol Infect 15:982-998

Sitheeque MA, Samaranayake LP (2003) Chronic hyperplastic candidiosis/candidiasis (candidal leukoplakia). Crit Rev Oral Biol Med 14:253-267

Spandole S, Cimponeriu D, Berca LM, Mihăescu G (2015) Human anelloviruses: an update of molecular, epidemiological and clinical aspects. Arch Virol 160:893-908 
Stanley MA (2012) Epithelial cell responses to infection with human papillomavirus. Clin Microbiol Rev 25:215-222

Stöppler MC, Straight SW, Tsao G, Schlegel R, McCance DJ (1996) The E5 gene of HPV-16 enhances keratinocyte immortalization by full-length DNA. Virology 223:251-254

Sugden B (2014) Epstein-Barr virus: the path from association to causality for a ubiquitous human pathogen. PLoS Biol 12(9):e1001939

Suzuki PS, de Oliveira KB, do Amaral Herrera AC, Borelli SD, Guembarovski RL, de Oliveira CEC, Oda JMM, Ozawa PMM, Watanabe MAE (2014) TT virus in peripheral blood cells from patients with Human Papillomavirus (HPV): investigating association with cervical carcinoma. Braz Arch Biol Technol 57:228-232

Szládek G, Juhász A, Kardos G, Szoke K, Major T, Sziklai I, Tar I, Márton I, Kónya J, Gergely L, Szarka K (2005) High co-prevalence of genogroup 1 TT virus and human papillomavirus is associated with poor clinical outcome of laryngeal carcinoma. J Clin Pathol 58:402-405

Tahara T, Arisawa T, Shibata T, Yamashita H, Yoshioka D, Hirata I (2009) Effect of promoter methylation of multidrug resistance 1 (MDR1) gene on gastric carcinogenesis. Anticancer Res 29:337-341

Takacs M, Banati F, Koroknai A, Segesdi J, Salamon D, Wolf H, Niller HH, Minarovits J (2010) Epigenetic regulation of latent Epstein-Barr virus promoters. Biochym Biophys Acta 1799:228-235

Thom K, Petrik J (2007) Progression towards AIDS leads to increased Torque teno virus and Torque teno minivirus titers in tissues of HIV infected individuals. J Med Virol 79:1-7

Tillonen J, Homann N, Rautio M, Jousimies-Somer H, Salaspuro M (1999) Role of yeasts in the salivary acetaldehyde production from ethanol among risk groups for ethanol-associated oral cavity cancer. Alcohol Clin Exp Res 23:1409-1415

Tsai S, Wear DJ, Shih JW, Lo SC (1995) Mycoplasmas and oncogenesis: persistent infection and multistage malignant transformation. Proc Natl Acad Sci U S A 92:10197-10201

Vasilyev EV, Trofimov DY, Tonevitsky AG, Ilinsky VV, Korostin DO, Rebrikov DV (2009) Torque Teno Virus
(TTV) distribution in healthy Russian population. Virol J 6:134-137

Venuti A, Paolini F, Nasir L, Corteggio A, Roperto S, Campo MS, Borzacchiello G (2011) Papillomavirus E5: the smallest oncoprotein with many functions. Mol Cancer 10:140-157

Vinokurova S, Wentzensen N, Kraus I, Klaes R, Driesch C, Melsheimer P, Kisseljov F, Dürst M, Schneider A, von Knebel Doeberitz M (2008) Typedependent integration frequency of human papillomavirus genomes in cervical lesions. Cancer Res 68:307-313

Warnakulasuriya S (2009) Global epidemiology of oral and oropharyngeal cancer. Oral Oncol 45:309-316

Westra HW (2009) The changing face of head and neck cancer in the 21st century: the impact of HPV on the epidemiology and pathology of oral cancer. Head Neck Pathol 3:78-81

Williams VM, Filippova M, Soto U, Duerksen-Hughes PJ (2011) HPV-DNA integration and carcinogenesis: putative roles for inflammation and oxidative stress. Future Virol 6:45-57

Wolf H, zur Hausen H, Becker V (1973) EBV viral genomes in epithelial nasopharyngeal carcinoma cells. Nat New Biol 244:245-247

Woods R Sr, O'Regan EM, Kennedy S, Martin C, O'Leary JJ, Timon C (2014) Role of human papillomavirus in oropharyngeal squamous cell carcinoma: a review. World J Clin Cases 2:172-193

Zhang B, Shih JW, Wear DJ, Tsai S, Lo SC (1997) Highlevel expression of H-ras and c-myc oncogenes in mycoplasma-mediated malignant cell transformation. Proc Soc Exp Biol Med 214:359-368

Zhong S, Yeo W, Lin CK, Lin XR, Tang MW, Johnson PJ (2001) Quantitative and genotypic analysis of TT virus infection in Chinese blood donors. Transfusion 41:1001-1007

Zignego AL, Ferri C, Pileri SA, Caini P, Bianchi FB, Italian Association of the Study of Liver Commission on Extrahepatic Manifestations of $\mathrm{HCV}$ infection (2007) Extrahepatic manifestations of Hepatitis C Virus infection: a general overview and guidelines for a clinical approach. Dig Liver Dis 39:2-17

zur Hausen H (2009) Papillomaviruses in the causation of human cancers - a brief historical account. Virology 384:260-265 